\title{
Calculation of Tin Atomic Data and Plasma Properties
}

prepared by

Energy Technology Division

Argonne National Laboratory 


\section{About Argonne National Laboratory}

Argonne is managed by The University of Chicago for the U.S. Department of Energy under contract W-31-109-Eng-38. The Laboratory's main facility is outside Chicago, at 9700 South Cass Avenue, Argonne, Illinois 60439. For information about Argonne and its pioneering science and technology programs, see www.anl.gov.

\section{Availability of This Report}

This report is available, at no cost, at http://www.osti.gov/bridge. It is also available on paper to U.S. Department of Energy and its contractors, for a processing fee, from:

U.S. Department of Energy

Office of Scientific and Technical Information

P.O. Box 62

Oak Ridge, TN 37831-0062

phone (865) 576-8401

fax (865) 576-5728

reports@adonis.osti.gov

\section{Disclaimer}

This report was prepared as an account of work sponsored by an agency of the United States Government. Neither the United States Government nor any agency thereof, nor The University of Chicago, nor any of their employees or officers, makes any warranty, express or implied, or assumes any legal liability or responsibility for the accuracy, completeness, or usefulness of any information, apparatus, product, or process disclosed, or represents that its use would not infringe privately owned rights. Reference herein to any specific commercial product, process, or service by trade name, trademark, manufacturer, or otherwise, does not necessarily constitute or imply its endorsement, recommendation, or favoring by the United States Government or any agency thereof. The views and opinions of document authors expressed herein do not necessarily state or reflect those of the United States Government or any agency thereof, Argonne National Laboratory, or The University of Chicago. 


\section{Calculation of Tin Atomic Data and Plasma Properties}

by

V. Morozov, V.Tolkach, and A. Hassanein

Energy Technology Division, Argonne National Laboratory

September 2004

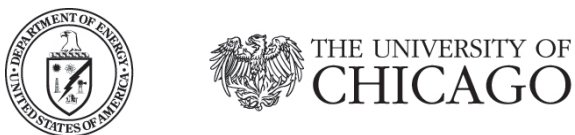

Argonne National Laboratory is managed by

The University of Chicago for the U.S. Department of Energy 


\section{CONTENTS}

ABSTRACT




\section{FIGURES}

Fig. 1: Concentrations of tin ions for various densities .......................................................5

Fig. 2: Calculations from equation of state for tin ions at various densities............................... 7

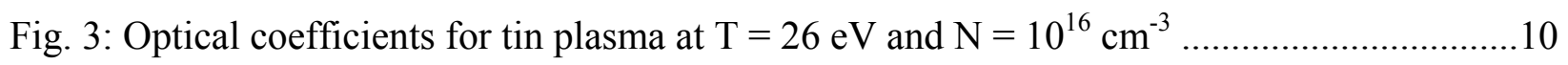

Fig. 4: Planck mean optical coefficients for tin plasma....................................................... 10

Fig. 5: Experimental data vs. calculation for Sn VIII $4 d^{7}-4 d^{6} 5 p^{1}$ transition..........................19

Fig. 6: Calculation of tin EUV transitions by the HEIGHTS-ATOM code................................20

Fig. 7: Calculation of tin EUV transitions by the Cowan code ................................................21

Fig. 8: Tin plasma optical coefficients in the EUV range ...................................................23

\section{TABLES}

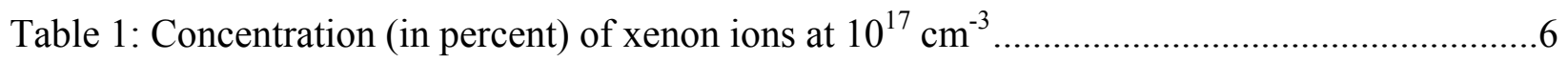

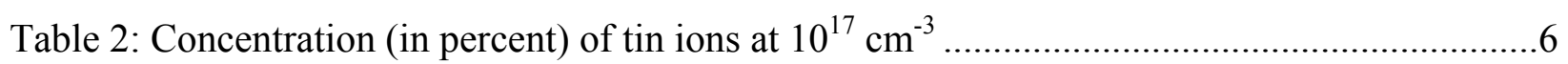

Table 3: Energies of Xe XI inner shells by $L S D$ HF and average term HF ..............................13

Table 4: Energies of Sn VIII inner shells by LSD HF and average term HF.............................13

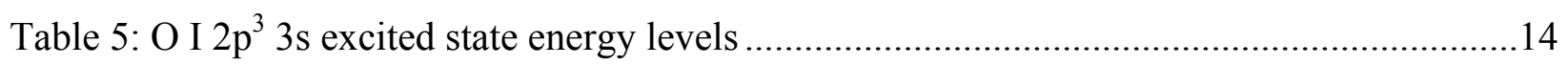

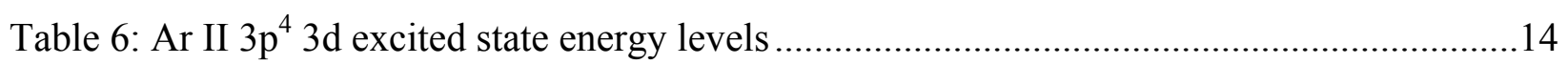

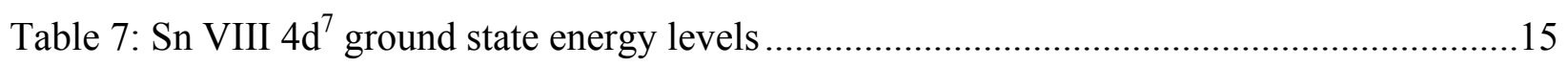

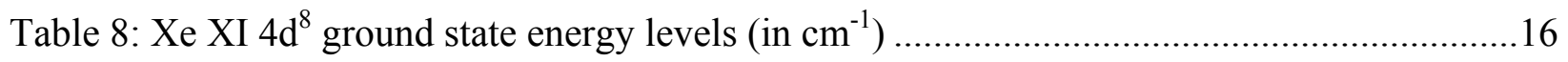

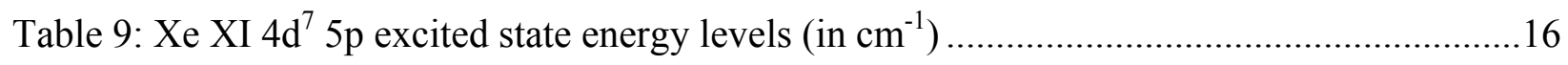

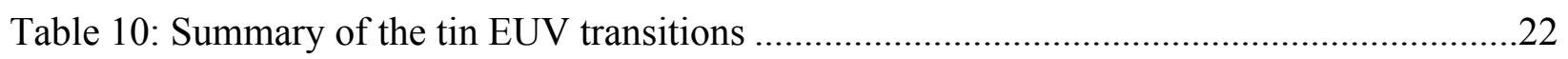




\begin{abstract}
This report reviews the major methods and techniques we use in generating basic atomic and plasma properties relevant to extreme ultraviolet (EUV) lithography applications. The basis of the work is the calculation of the atomic energy levels, transitions probabilities, and other atomic data by various methods, which differ in accuracy, completeness, and complication. Later on, we calculate the populations of atomic levels and ion states in plasmas by means of the collision-radiation equilibrium (CRE) model. The results of the CRE model are used as input to the thermodynamic functions, such as pressure and temperature from the internal energy and density (equation of state), electric resistance, thermal conduction, and other plasma properties. In addition, optical coefficients, such as emission and absorption coefficients, are generated to resolve a radiation transport equation (RTE). The capabilities of our approach are demonstrated by generating the required atomic and plasma properties for tin ions and plasma within the EUV region near $13.5 \mathrm{~nm}$.
\end{abstract}

\title{
INTRODUCTION
}

To meet the requirements of the Intel Lithography Roadmap goals for high volume manufacturing in the future [1] and International SEMATECH's EUV Source Program goals [2], the EUV source is required to have a power of $80-120 \mathrm{~W}$ at $13.5 \mathrm{~nm}$ ( $2 \%$ bandwidth). Various laser produced plasma (LPP) and gas discharge produced plasma (DPP) devices are under development and investigation by different research groups. Both types of EUV sources have advantages and disadvantages. At present, none of the current EUV sources can deliver enough power levels demanded by commercial chip manufacturers.

The efficiency of generating EUV radiation is the key factor in successful development of the source. The leading EUV groups are using a variety of scientific and engineering approaches to maximize EUV brightness from their devices. A common technique is the increase of the optimized overall conversion efficiency of a device, because it minimizes the required input power for a required EUV output. Because many physical processes are involved, and many technical problems need to be solved, when optimizing a particular EUV device, only computer modeling can generate a complete picture at a reasonable pace.

We are developing an integrated model to simulate the environment of the EUV source and optimize the output of the source. The model describes the hydrodynamic and optical processes that occur in EUV devices. It takes into account plasma evolution and magnetohydrodynamic (MHD) processes as well as photon radiation transport. It uses the total variation diminishing scheme in the Lax-Friedrich formulation for the description of magnetic compression and diffusion in a cylindrical geometry. Also under development are models for opacity calculations: a collisional radiation equilibrium model, a self-consistent field model with Auger processes, and a nonstationary kinetic model. Radiation transport for both continuum and lines with detailed spectral profiles is taken into account. The developed models are being integrated into the HEIGHTS-EUV computer simulation package [3, 4]. Being self-consistent, 
the HEIGHTS-EUV package can generate all required information and is completely independent of the data from other external packages. Furthermore, experimentally (or numerically) obtained reliable data can be incorporated into HEIGHTS-EUV to increase the overall accuracy and efficiency of the simulation results.

The focus of this report is the major methods and techniques we use in our HEIGHTSATOM code to generate basic atomic and plasma properties. Based upon accurately generated atomic energy levels, transition probabilities, and other atomic data, we can calculate the populations of atomic levels and ion states in plasma by means of the CRE model. In turn, the results of the CRE model are used as input to thermodynamic functions, such as those for pressure and temperature from the internal energy and density (equation of state), electric resistance, thermal conduction, and other plasma properties. Optical coefficients are also generated to resolve the RTE. Combining the generated properties with an appropriate description of an EUV device in terms of the MHD boundary conditions, we are able to simulate the dynamics of the device and its total radiation output. The accuracy and completeness of atomic data are key factors in the successful numerical simulation of the EUV device.

This report contains the results of our computer simulation of the tin atomic data and plasma properties in tabulated form. The calculation of atomic properties by the Hartree-FockSlater (HFS) approximation is described in the first section. Section 2 is devoted to the description of the CRE model, which is used to obtain the populations of the atomic levels and the ion composition of the plasma. The calculation of thermodynamic plasma properties and the equation of state are described in the third section. The resolution of the radiation transport equation in a wide spectral range depends upon the completeness of the optical coefficients, which are discussed in Section 4. The Planck mean opacities are presented in Section 5. The next three sections are primarily dedicated to calculating highly accurate EUV information for tin. Section 6 discusses the limitations of using the simplified methods for generating EUV atomic data and describes the advanced $L S$-dependent Hartree-Fock ( $L S D$ HF) atomic method. Detailed EUV transitions are presented in Section 7. Finally, Section 8 presents calculations of tin EUV opacities with very high resolution.

\section{HFS ENERGY LEVELS AND OTHER DATA}

In modeling the dynamics and the output of an EUV source, one needs to distinguish two aspects. First, in solving the hydrodynamic part of the problem, the plasma internal energy must be corrected and re-distributed accordingly to the radiation transport. A key element in this process is the completeness of the radiant energy redistribution in the whole plasma domain within the very broad spectral range of participating photons. The second aspect is the detailed calculation of the effective radiation of the EUV source within the operating energy range of $13.5 \pm 2 \% \mathrm{~nm}$. In this case, the calculation of the radiation transport must be oriented to the accurate accounting of only those photons, whose energies are within the narrow EUV range.

The simulation of the dynamics of the plasma evolution typically involves a wide range

of temperature and density values and a very complicated ionic structure. From the atomic physics viewpoint, detailed resolution of each possible level for each possible ion (and 
consequently, each electronic transition in the ion) in a wide range of temperatures, densities, and energies is enormously laborious, especially when one is accounting for the fine structure of each split level. The difficulties come from the very large number of atomic terms and split levels. For example, to split a $d^{5}$ shell, one needs to account for 16 terms and 37 levels. The $f^{7}$ shell will have 119 terms and 327 levels. In approximation of configurations, the transition $4 d^{n}-4 d^{n-1} 4 f$ will only have one strong line, while splitting the shells into levels replaces this line by several hundred weak lines. Knowing that the total transition strength is unchanged, the radiation in strong lines may be collapsed for a dense plasma, while the radiation in weak lines stays optically transparent. The line splitting may dramatically influence the total hydrodynamic behavior of a plasma through the radiation transport mechanism [5].

The results of theoretical approximations of atomic data strongly depend on the chosen theoretical models [6 - 8], and this is particularly true for intermediate- and high-Z materials. To describe a spherically symmetrical quantum system, the self-consistent field methods, such as Dirac-Fock (DF) or Hartree-Fock (HF) methods, are believed to be the most effective. However, the very large number of ions and their atomic levels and transitions involved into the plasma computation, limits their applicability. In consistently solving the MHD equations and the RTE, the plasma properties, opacities, equation of state, and ion concentrations can be obtained from the structures of atomic levels and transition probabilities, calculated by simplified methods. One such method is the HFS approximation [9], which allows the determination of the energies and other atomic characteristics for each $n l$-configuration of each ion that might exist in the plasma. The simplification of the method normally results in the shift of some spectral lines from their true places by several percent, which is not problematic in determining the integral radiation flux. Nevertheless, we cannot neglect the fine structure of open shells, and have thus combined the HFS methods with the splitting of the energy levels and spectral lines by means of the Racah theory of angular momentum within the framework of perturbation theory $[10,11,5]$.

The accuracy of the HFS model is typically within several percents for the split levels. However, this accuracy is not good enough for the second part of our project, which involves determining the EUV output of the source within the very narrow $2 \%$ bandwidth. [To obtain a spectroscopic accuracy of the EUV optical coefficients, the atomic data are calculated by the $L S$ dependent ( $L S D)$ HF method, as discussed in Section 6.]

In the HFS method the potential of direct electron interaction is calculated from the radial wavefunctions of participating electrons, and exchange interactions are averaged in the form of the exchange potential $V_{e x}$. The radial wavefunction of an atom can be represented as the product of radial wavefunctions $P_{n l}(r)$ of the electrons. It is assumed that all $q_{n l}$ equivalent electrons have the same radial wavefunction:

$$
\begin{aligned}
& \left(\frac{d^{2}}{d r^{2}}+\frac{2 Z(r)}{r}+V_{e x}(r)-\frac{l(l+1)}{r^{2}}+\varepsilon_{n l}\right) P_{n l}(r)=0, \\
& \frac{Z(r)}{r}=\int_{r}^{\infty} \frac{Z_{0}-\int_{0}^{n_{1}} \rho(x) d x}{r_{1}^{2}} d r_{1}, \quad V_{e x}(r)=-\frac{3}{2}\left(\frac{3}{\pi} \rho\right)^{\frac{1}{3}}, \quad \rho(r)=\sum_{n l} q_{n l} P_{n l}^{2}(r) .
\end{aligned}
$$


Traditionally, in atomic physics, the energies are expressed in Ry, and distances are in $a_{0}$ or Bohr units. In the above equations, $\varepsilon_{n l}$ is binding energy of the electron, $Z(r)$ is the effective charge of the ion field, $Z_{0}$ is the nuclear charge, and $\rho(r)$ is the electron density.

From solution of the HFS equations, one may find one radial wavefunction for each shell of the atom or ion. We normally calculate the functions for each ion, starting from the neutral and ending with the totally ionized core. Obtained wavefunctions are used to later calculate relativistic corrections, transition wavelengths, and dipole transitions from the ground state to the highly excited state (principal quantum number may reach up to 10), allowed by the selection rules. Discrete transitions from inner shells with energies limited to the value dependent on ionization potential were also taken into account. Photoionization crosssections were calculated for all inner and excited states. Detailed spin-orbit splitting of non-filled shells was implemented outside the HFS, but within the CRE model, by using Slater integrals $F^{k}, G^{k}$, and constants of spin-orbit splitting $\xi^{k}$ as described in Ref. 5.

\section{ION STATES AND POPULATIONS OF ATOMIC LEVELS}

To describe the populations of atomic levels, we utilize the CRE model, which is equally applicable for low, medium, and high temperature ranges. The CRE model accounts for the excitation and ionization processes that can take place in a plasma. The fact that the CRE model considers the transitions between all atomic levels is of particular importance. Nonlocal effects are accounted for in the form of an escape factor $[12,13]$, which neglects by photoexcitation in continuum and reduces the strength of spontaneous transitions. Such an approximation fairly describes the plasma behavior in the condition of absence of an external source of hard radiation.

The plasma ionization state and population levels $n_{i}$ for a prescribed set of temperatures and densities are calculated according to the system of kinetic equations in stationary form:

$$
\frac{d n_{i}}{d t}=-n_{i} \sum_{j \neq i} K_{i j}+\sum_{i \neq j} n_{j} K_{j i}=0 .
$$

The population of atomic level $i$ is determined by the set of transitions from this level to other levels $j$ with transition rates $K_{i j}$, as well as transitions from other levels $j$ to this level $i$ with transition rates $K_{j i}$. One equation is written for each atomic level. If level $i$ defines the ground state, then the population of this atomic level gives the concentration of the ion in the plasma. Impact-electron excitation and de-excitation, impact electron ionization, three-body recombination, spontaneous transition, and photo- and di-electronic recombination are included in calculation of total rates of electron transition. From the known population of levels, ion and electron concentrations $N_{i}$ and $N_{e}$ are defined for a given temperature.

A numerical simulation of concentration of tin ions calculated by the CRE model with splitting HFS atomic levels is presented in Fig. 1 for various plasma densities. Due to ionization of the $d$-shell, the ions change very quickly when temperature increases. This condition results in a low concentration of the major ion, hardly larger than $50 \%$ at best. At the same time, the clear advantage of tin as an EUV working element is that several ions are EUV productive, 
which noticeably widen the range of the required temperature in the device. This situation is especially true at low density, when EUV emitting ions are appearing at slightly higher temperature and not changing as fast.
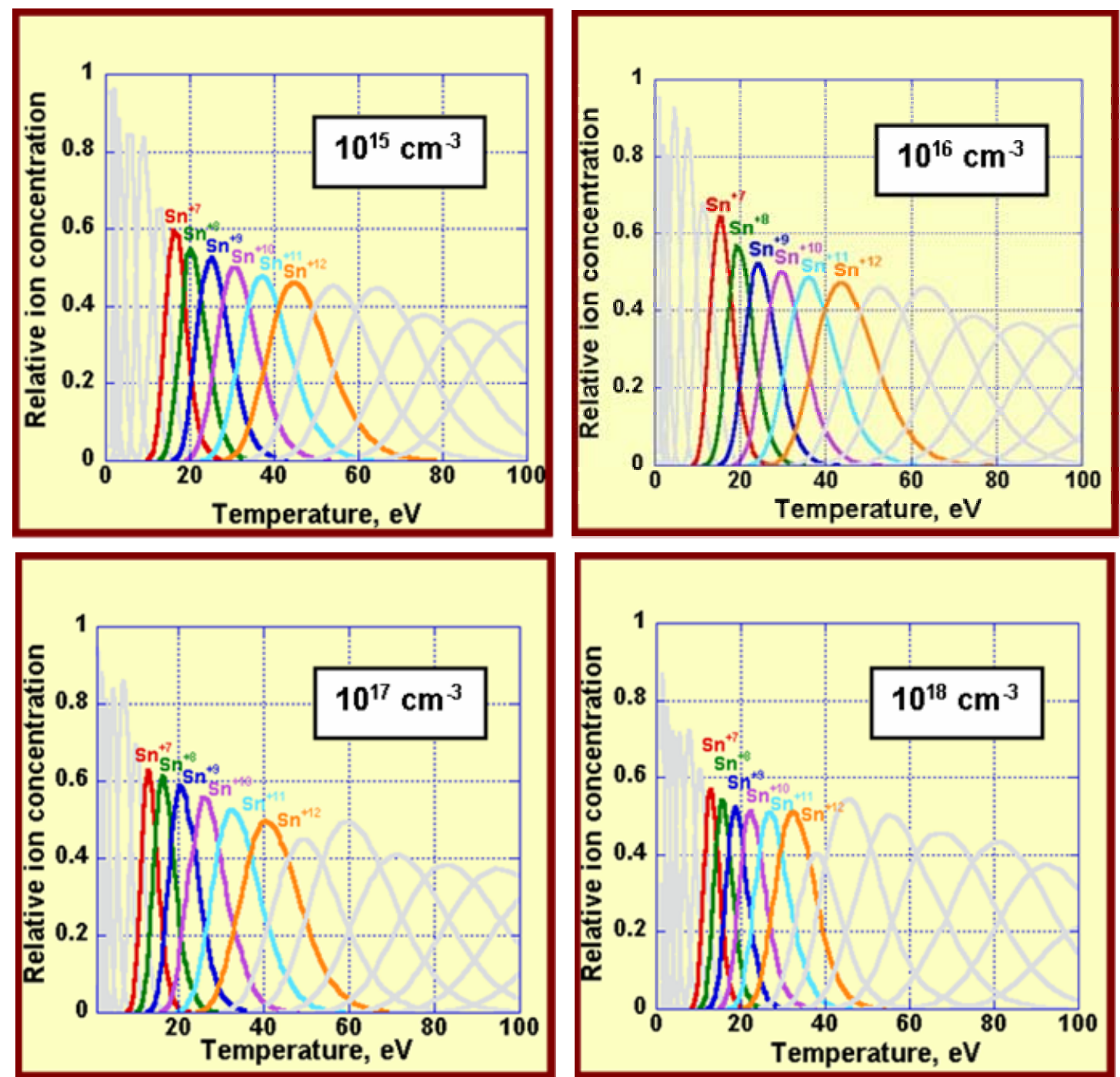

Fig. 1: Concentrations of tin ions for various densities

Our calculations can be validated and benchmarked by comparison to the results of other authors. Accordingly to the results calculated by the CRE model and reported in [14], the concentrations of xenon ions at $n_{e}=10^{17} \mathrm{~cm}^{-3}$ and $T_{e}=32 \mathrm{eV}$ were $20 \% \mathrm{Xe}+9,25 \% \mathrm{Xe}+10$, $20 \% \mathrm{Xe}+11$, and $13 \% \mathrm{Xe}+12$, as shown by gray shading in the second column of Table 1 . As seen from the other data in the table, our calculations generally agree with these figures, except that we obtained a twice larger value at $T_{e}=31 \mathrm{eV}$ for the concentration of $\mathrm{Xe}+10$ ions. We tend to rely on nearly $45 \%$ concentration of $\mathrm{Xe}+10$, which would fix the total concentration of all ions to $100 \%$ and be more realistic in terms of the relative concentration of the major ion to the others.

The authors of Ref. 14 also reported ion concentrations at similar temperature and density 
for a tin plasma to be $15 \% \mathrm{Sn}+8,40 \% \mathrm{Sn}+9,30 \% \mathrm{Sn}+10$, and $15 \% \mathrm{Sn}+11$. As shown in column 2 of Table 2, these results are similar to our computations for $T_{e}=29 \mathrm{eV}$. Results of the tables generally confirm the very complex composite nature of xenon and tin plasmas, where at the same conditions one may expect to find up to seven ion species.

Table 1: Concentration (in percent) of xenon ions at $10^{17} \mathrm{~cm}^{-3}$. Top two rows give temperature and plasma density. Shaded data from Ref. 14.

\begin{tabular}{|c|c|c|c|c|c|c|c|c|c|c|}
\hline $\mathrm{T}_{\mathrm{e}}, \mathrm{eV}$ & 32 & 30.8 & 31.0 & 31.2 & 31.4 & 31.6 & 31.8 & 32.0 & 32.2 & 32.4 \\
\hline$\rho_{\mathrm{e}}, \mathrm{g} / \mathrm{cm}^{3}$ & & $2.19 \times 10^{-6}$ & $2.18 \times 10^{-6}$ & $2.17 \times 10^{-6}$ & $2.16 \times 10^{-6}$ & $2.16 \times 10^{-6}$ & $2.15 \times 10^{-6}$ & $2.14 \times 10^{-6}$ & $2.13 \times 10^{-6}$ & $2.13 \times 10^{-6}$ \\
\hline $\mathrm{Xe}+7$ & & 0.02 & 0.02 & 0.02 & 0.01 & 0.01 & 0.01 & 0.01 & 0.01 & 0.01 \\
\hline $\mathrm{Xe}+8$ & & 2.49 & 2.25 & 2.04 & 1.84 & 1.66 & 1.50 & 1.35 & 1.22 & 1.10 \\
\hline $\mathrm{Xe}+9$ & 20 & 24.15 & 22.90 & 21.69 & 20.51 & 19.37 & 18.29 & 17.23 & 16.22 & 15.25 \\
\hline $\mathrm{Xe}+10$ & 25 & 49.08 & 49.03 & 48.88 & 48.63 & 48.30 & 47.91 & 47.42 & 46.86 & 46.22 \\
\hline $\mathrm{Xe}+11$ & 20 & 22.07 & 23.34 & 24.62 & 25.91 & 27.19 & 28.49 & 29.76 & 31.02 & 32.26 \\
\hline $\mathrm{Xe}+12$ & 13 & 2.14 & 2.40 & 2.70 & 3.02 & 3.36 & 3.70 & 4.10 & 4.53 & 5.00 \\
\hline $\mathrm{Xe}+13$ & & 0.04 & 0.05 & 0.06 & 0.07 & 0.09 & 0.10 & 0.12 & 0.15 & 0.17 \\
\hline
\end{tabular}

Table 2: Concentration (in percent) of tin ions at $10^{17} \mathrm{~cm}^{-3}$. Top two rows give temperature and plasma density. Shaded data from Ref. 14.

\begin{tabular}{|c|c|c|c|c|c|c|c|c|}
\hline $\mathrm{T}_{\mathrm{e}}, \mathrm{eV}$ & 32 & 25 & 28 & 29 & 30 & 31 & 32 & 35 \\
\hline$\rho_{\mathrm{e}}, \mathrm{g} / \mathrm{cm}^{3}$ & & $2.37 \times 10^{-6}$ & $2.19 \times 10^{-6}$ & $2.14 \times 10^{-6}$ & $2.09 \times 10^{-6}$ & $2.05 \times 10^{-6}$ & $2.01 \times 10^{-6}$ & $1.91 \times 10^{-6}$ \\
\hline \hline $\mathrm{Sn}+7$ & & 12.42 & 2.85 & 1.64 & 0.92 & 0.51 & 0.28 & 0.04 \\
\hline $\mathrm{Sn}+8$ & 15 & 40.58 & 20.60 & 14.98 & 10.50 & 7.15 & 4.74 & 1.23 \\
\hline $\mathrm{Sn}+9$ & 40 & 37.07 & 45.50 & 42.80 & 38.25 & 32.73 & 26.96 & 12.58 \\
\hline $\mathrm{Sn}+10$ & 30 & 8.27 & 26.94 & 33.69 & 39.35 & 43.32 & 45.30 & 40.18 \\
\hline $\mathrm{Sn}+11$ & 15 & 0.40 & 3.85 & 6.59 & 10.34 & 15.02 & 20.41 & 36.66 \\
\hline $\mathrm{Sn}+12$ & & 0.00 & 0.12 & 0.29 & 0.63 & 1.25 & 2.26 & 8.80 \\
\hline
\end{tabular}




\section{THERMODYNAMIC PLASMA PROPERTIES AND EQUATION OF STATE}

After electron $N_{e}$ and ion $N_{i}$ concentrations are found by the CRE model, they can be substituted into a set of equations of state. Generally, the two-temperature approximation for pressure $p$ contains the correspondent terms for kinetic energy of ions and electrons. The equation for internal energy $e_{\text {int }}$ contains the terms for ionization and excitation of electrons:

$$
\begin{aligned}
& P=k T_{i} \sum_{i} N_{i}+k T_{e} N_{e}, \\
& e_{\text {int }}=\frac{3 k T_{e} N_{e}}{2 \rho}+\frac{3 k T_{i} \sum_{i} N_{i}}{2 \rho}+\frac{1}{\rho} \sum_{i} N_{i}\left(\left(\sum_{i=1} I_{i}\right)+\sum_{j} \frac{\varepsilon_{j i} N_{j i}}{N_{i}}\right) .
\end{aligned}
$$

Here we use traditional notation for Boltzmann's constant $k$, ionization potential $I_{i}$ of the atomic level $i$, plasma density $\rho$, excitation energy of level $j$ to level $i \varepsilon_{j i}$, electron and ion temperatures $T_{e}, T_{i}$. Results of these calculations are presented in Fig. 2.
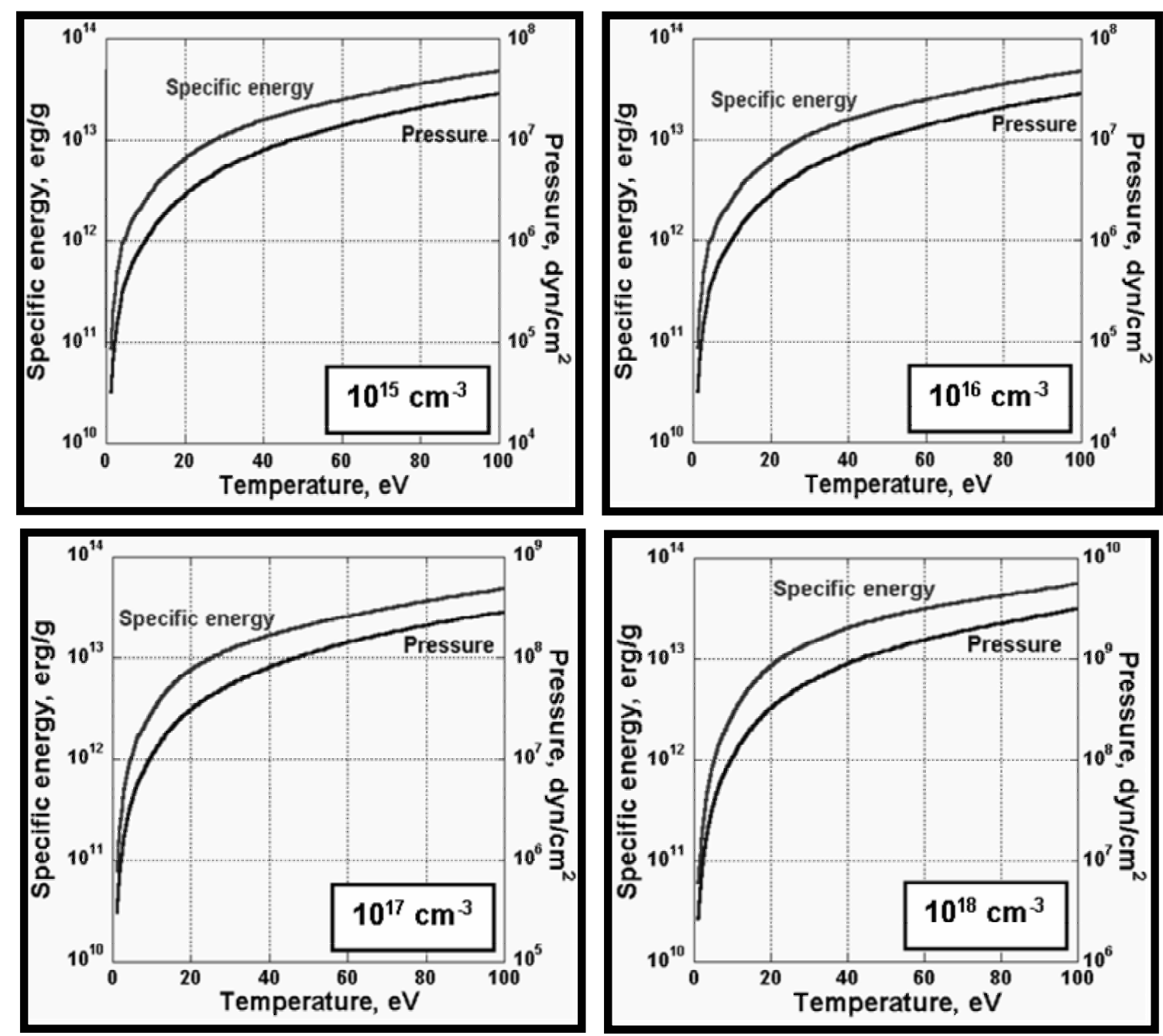

Fig. 2: Calculations from equation of state for tin ions at various densities 
Reciprocal to the resistivity $\eta$, the electrical conductivity $\sigma$ is found as the sum of conductivities defined by the electron scattering $\sigma_{c}$ and $\sigma_{n}$ on charged and neutral particles.

$$
\eta=\frac{1}{\sigma}=\frac{1}{\sigma_{c}}+\frac{1}{\sigma_{n}} ; \quad \sigma_{c}=\frac{4 \sqrt{2}}{\pi \sqrt{\pi}} \frac{\left(k T_{e}\right)^{3 / 2} \beta}{e^{2} Z \Lambda} ; \quad \sigma_{n}=\frac{3}{2} \sqrt{\frac{2}{\pi}} \frac{N_{e} e^{2}}{\sqrt{m_{e} k T_{e}} N_{0} s_{0}} .
$$

Here we use standard notation for Coulomb logarithm $\Lambda$, concentration of neutral atom $N_{0}$, electron charge $e$, and mean ion charge $Z$. Parameter $\beta$ is an electron-electron scattering correction [15]. Empirical values of transport crosssections $s_{0}$ are taken from [16].

\section{DETAILED SET OPACITIES IN WIDE ENERGY RANGE}

The electronic transitions and their accompanying absorption and emission of photons are subdivided into three types: bremsstrahlung; photoionization from ground, excited, and inner levels; and discrete transitions. The latter is approximated in the form of dipole transitions and includes transitions between ground and excited states, transitions between excited states, and partly the transitions from inner shells. Because of its importance, the profiles of spectral lines are processed very carefully by means of all major broadening mechanisms, such as radiation, Stark, Doppler, and resonance broadenings [17].

The total absorption coefficient $\kappa_{a b s}$ is calculated as a sum of absorption coefficients for free-free $\kappa_{f f}$, bound-free $\kappa_{b f}$, and bound-bound $\kappa_{b b}$ radiation transitions weighed to the value of the population levels:

$$
\begin{aligned}
& \kappa_{f f}(T, \rho, \hbar \omega)=\sum_{i} \sigma_{i}(T, \hbar \omega) n_{i}(T, \rho) n_{e}(T, \rho)(1-\exp (-\hbar \omega / k T)), \\
& \kappa_{b f}(T, \rho, \hbar \omega)=\sum_{i} \sum_{j} \sigma_{i j}(\hbar \omega) N_{i j}(T, \rho), \\
& \kappa_{b b}(T, \rho, \hbar \omega)=\sum_{i} \sum_{j, k} \frac{\pi e^{2}}{m_{e} c} f_{j k} \Phi(T, \rho, \omega) N_{i j}(T, \rho) .
\end{aligned}
$$

Knowing the crosssections of inverse processes, the total emission coefficient $\kappa_{e m i}$ is calculated by similar formulae. The values of electron density $n_{e}$ and ion density $n_{i}$ are calculated as described in previous section. The oscillator strengths $f_{j k}$, crosssections for photo absorption $\sigma_{i}$, crosssections for photoionization $\sigma_{i j}$, and line profile $\Phi$ are given elsewhere [5, $6,8]$.

As shown earlier [5, 18], accounting for electrostatic and spin-orbit splitting of shells and spectral lines considerably influences the dynamics of the energy balance in the plasma. A method of calculating the optical coefficients for high-Z plasma was developed and implemented $[5,19]$ by means of the CRE model. The important feature of this method is the joint use of HFS atomic data and Racah techniques of angular moments. This feature makes possible the use of comparatively unsophisticated methods to consider the complex electronic structure of each 
participating ion, and the complicated splitting of each configuration into terms (using the $L S$ coupling approximation), over a wide range of spectral frequencies, and in the expected range of temperatures and densities. By this technique, the detailed emission and absorption spectra are initially calculated over a very complete spectral frequency scale (up to 100,000 points) for the expected range of MHD values. The width of the frequency interval was comparable to the Doppler width of the strongest spectral lines, which provides a satisfactory resolution of the line profiles. In Fig. 3 we present calculated emission and absorption coefficients for the tin plasma at typical temperature and density $\left(26 \mathrm{eV}\right.$ and $\left.10^{16} \mathrm{~cm}^{-3}\right)$.

\section{PLANCK GROUP AVERAGED OPACTITES IN WIDE ENERGY RANGE}

Because of the large size of the generated opacity tables, the practical use of such detailed data is not convenient, and the emission and absorption coefficients are thus averaged in spectral groups. A rigorous theory for averaging the opacities within a group of frequencies does not exist. Such an averaging procedure is considered correct only when the absorption coefficient is constant within the group, or the optical thickness of each line is very small, and the absorption becomes a linear-like function from the frequency. This situation is only possible for a continuum, but even in that case, every photoionization threshold must become a boundary of a group. It becomes even more complicated for the lined spectrum, when the absorption coefficient often drops several orders of magnitude within a very limited frequency interval, say, from the center of a very strong spectral line to the wings of the same line, and the center of the line is optically thick. Moreover, the temperature and density values may vary. This change leads to spectral lines for the other ions, along with changes in the width of the existing lines.

From a practical viewpoint, an organized selection of the strongest lines is a reasonable way to describe the optical coefficients within the most important hydrodynamic areas for typical temperature and density values. The other lines are averaged within broad groups. Unfortunately, the primary goal of the numerical simulation is the determination of the typical hydrodynamic parameters within the important areas of the plasma domain!

For a uniform isothermal plasma, the optical thickness of a spectral line is determined by multiplying the absorption coefficient of the line by the linear dimension of the plasma $\tau(\varepsilon)=\kappa_{\text {abs }}(\varepsilon) \cdot l$. In the case of a nonuniform nonisothermal plasma, this definition is generalized as $\tau(\varepsilon)=\int \kappa_{\text {abs }}(T, \rho, \varepsilon) d l$ over the interval $\Delta l$, where the ion exists emitting with frequency $\varepsilon$.

The borders of the groups are calculated from the following considerations. First, the width of a group $\Delta \varepsilon$ should never exceed $\alpha T$, where $T$ is the plasma temperature, and $\alpha$ is a chosen parameter for averaging. This condition provides the smooth averaging of opacities in the continuum region, where the averaging is normally performed within the broad groups, and the optical thickness is much less than one. Second, invariability of the optical coefficient is required for a chosen value $\beta$ within the group. This condition provides a very specified resolution of the spectral lines with the optical thickness nearly at unity, and the wings of the lines with the optical thickness greater than unity. A final consideration is that all those 
frequencies which belong in the detailed spectrum to a line with $\tau(\varepsilon) \geq 1$ are also included in the domain of the groups. This condition provides a very thorough resolution of the strong lines in the averaging spectrum. By variation of the parameters $\alpha$ and $\beta$, several group mean opacities are generated with different levels of completeness and detail.
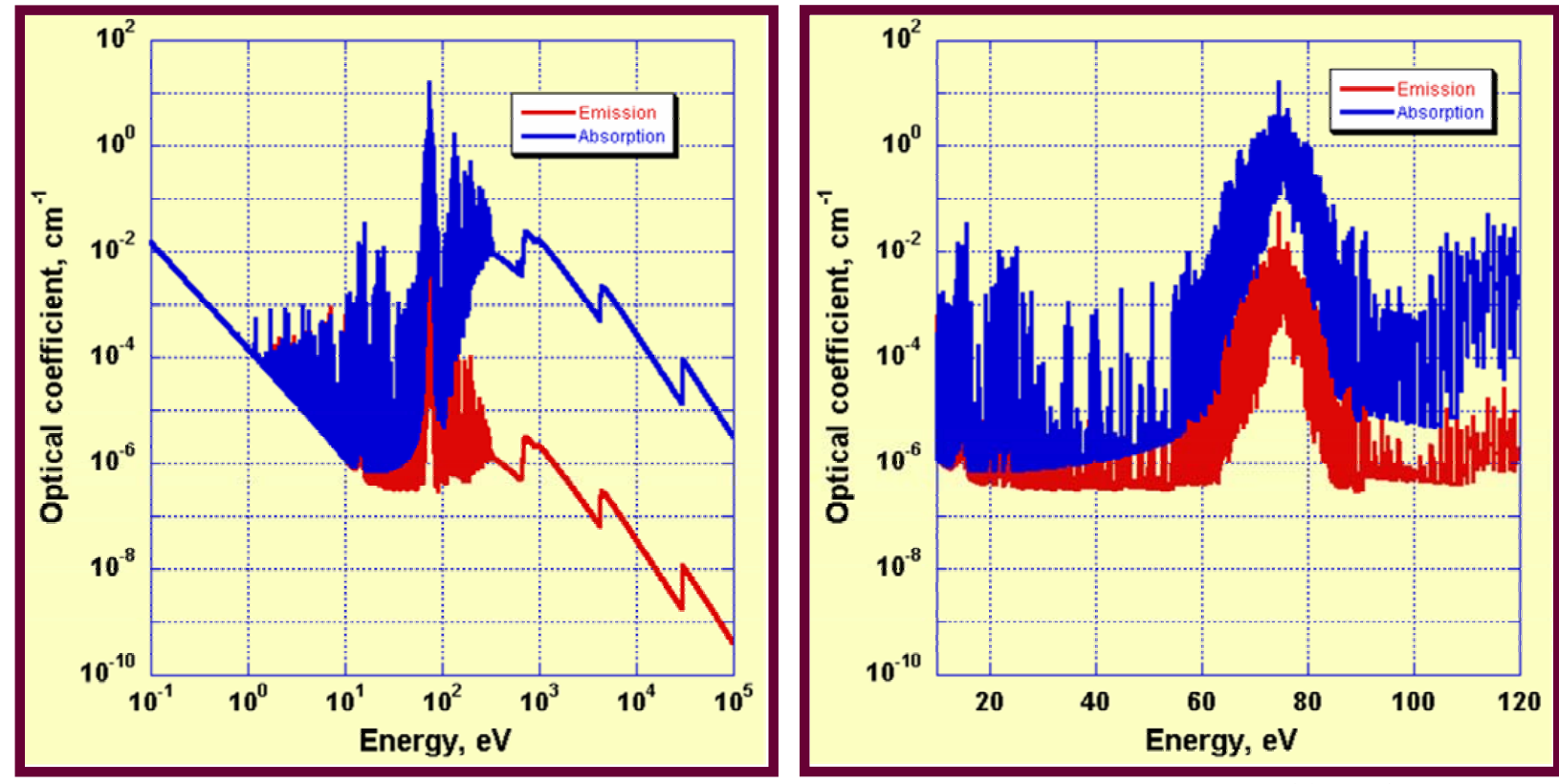

Fig. 3: Optical coefficients for tin plasma at $T=26 \mathrm{eV}$ and $\mathrm{N}=10^{16} \mathrm{~cm}^{-3}$
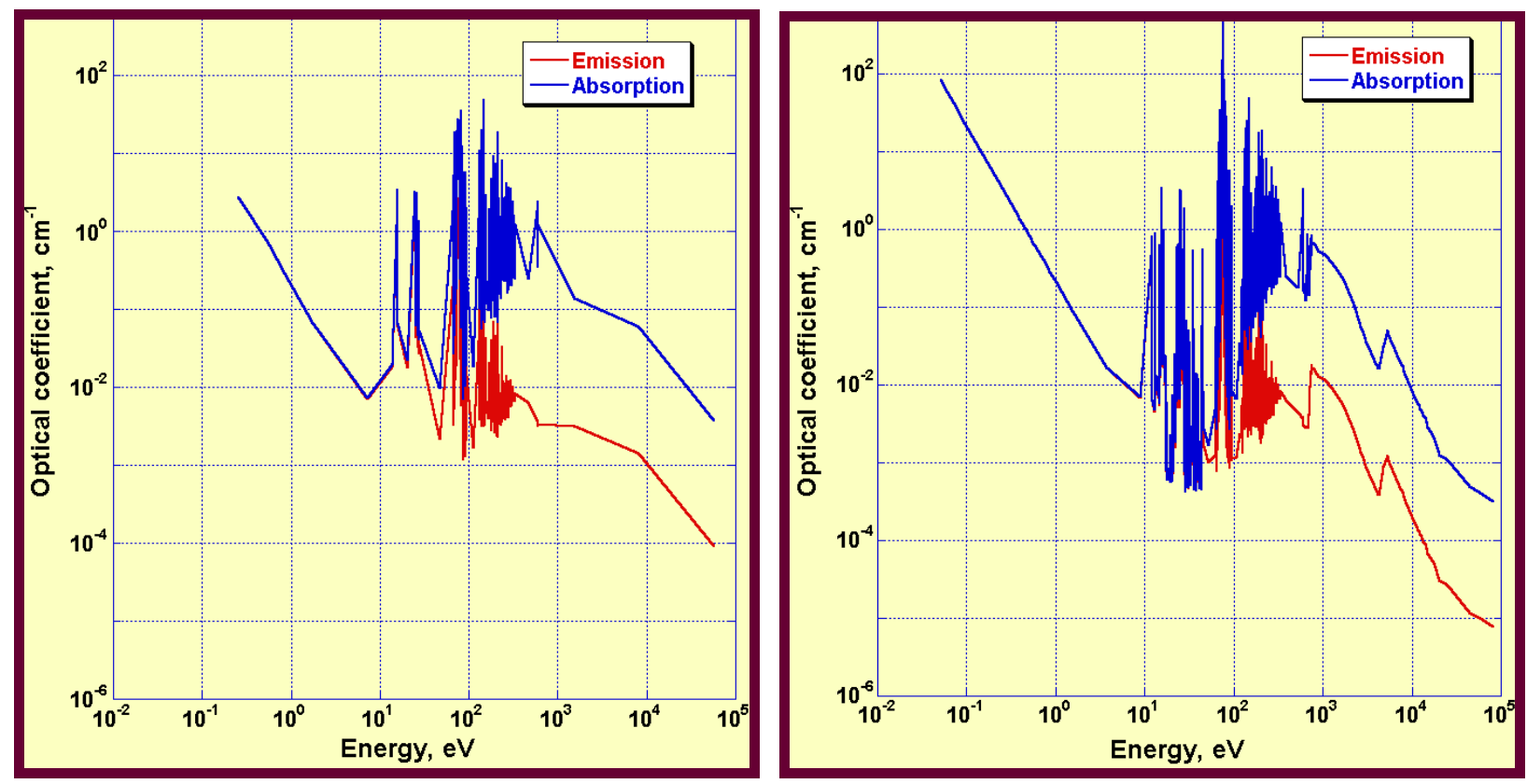

Fig. 4: Planck mean optical coefficients for tin plasma, averaged over 691 (left) and 3240 (right) groups 
Based upon several recent studies [3, 4], it is supposed that the maximal radiation flux corresponds to the moment of pinch formation. Typical tin pinch parameters are the following: the temperature is close to $25 \mathrm{eV}$, the density is $3 \cdot 10^{17} \mathrm{~cm}^{-3}$, the spectral range in radiation energies varies from $5 \mathrm{eV}$ to $250 \mathrm{eV}$, and the average optical plasma thickness is $1 \mathrm{~cm}$. Using these parameters, we have generated a basic set of opacities, averaged within 691 spectral groups. Taking a wider set of temperature and density pairs, we have generated the optimal scale of spectral groups. Combining all scales, the resulting energy scale has a total of 3240 energy groups. Results of the Planck mean absorption and emission coefficients for the mentioned temperature and density values are shown in Fig. 4 above. As the next step, the output of the self-consistent hydrodynamic/radiation transport calculations with these opacities can be used for further improvements in the quality of the coefficients.

\section{HF ENERGY LEVELS AND OTHER DATA FOR EUV EMITTING TIN ION SPECIES}

The accuracy of the HFS model is typically within several percents for the split levels, which is insufficient for the narrow $2 \%$ bandwidth. To obtain a higher accuracy of the EUV optical coefficients, we use the $L S D$ HF method, which is more accurate but significantly more advanced and difficult to implement. Strictly speaking, the HF method encompasses several methods for calculation of various atomic structures. Being applied to the same atomic system, each modification of the HF method can produce different results, so the choice of the appropriate method is very important in terms of the overall accuracy of the calculations.

In the HF method the wavefunctions, eigenvalues, and total energy of an atom are found from the variational principle for the well-known Schrödinger equation:

$$
\hat{H} \Psi=E \Psi,
$$

where $\hat{H}$ is a Hamiltonian of the atom, including the interaction of each electron with the nucleus and the other electrons; $E$ is the total energy of an atom; and $\Psi$ is the atomic wavefunction, which is expressed through the atomic orbitals $\varphi_{i}\left(r_{j}\right) \equiv \varphi_{n_{i} l_{i}}\left(r_{j}\right)$ as

$$
\Psi=\left|\begin{array}{cccc}
\varphi_{1}\left(r_{1}\right) & \varphi_{1}\left(r_{2}\right) & \cdots & \varphi_{1}\left(r_{n}\right) \\
\varphi_{2}\left(r_{1}\right) & \varphi_{2}\left(r_{2}\right) & \cdots & \varphi_{2}\left(r_{n}\right) \\
\cdots & \cdots & \cdots & \cdots \\
\varphi_{n}\left(r_{1}\right) & \varphi_{2}\left(r_{2}\right) & \cdots & \varphi_{n}\left(r_{n}\right)
\end{array}\right| .
$$

The number of the atomic orbitals is determined by the number of shells in the atom. For instance, the tin atom has $1 s^{2} 2 s^{2} 2 p^{6} 3 s^{2} 3 p^{6} 3 d^{10} 4 s^{2} 4 p^{6} 4 d^{10} 5 s^{2} 5 p^{2}$ ground state, or 11 orbitals. Each orbital is found from the system of integral-differential equations for functions $\varphi_{n l}$ : 


$$
\begin{aligned}
& \left\{-\frac{1}{2} \frac{d^{2}}{d r^{2}}+\frac{l(l+1)}{2 r^{2}}-\frac{Z}{r}+\frac{2}{N_{n l}} \sum_{x} f_{x}(n l) y_{n l, n l}^{x}(r)+\frac{1}{N_{n l}} \sum_{n^{\prime} l^{\prime}} \sum_{x} \alpha_{x}\left(n l, n^{\prime} l^{\prime}\right) y_{n^{\prime} l^{\prime}, n^{\prime} l^{\prime}}^{x}(r)-\varepsilon_{n l}\right\} \varphi_{n l}(r)- \\
& -\frac{1}{N_{n l}} \sum_{n^{\prime} l^{\prime} l^{\prime}} \sum_{x} \beta_{x}\left(n l, n^{\prime} l^{\prime}\right) y_{n^{\prime} l^{\prime}, n l}^{x}(r) \varphi_{n^{\prime} l^{\prime}}(r)-\sum_{n^{\prime}} \varepsilon_{n l, n^{\prime} l^{\prime}} \varphi_{n^{\prime} l^{\prime}}(r)=0 .
\end{aligned}
$$

The expressions for the radial integrals $y_{n^{\prime} l, n l}^{x}(r)$, as well as the potentials $f_{x} y_{n l, n l}^{x}(r)$, $\alpha_{x} y_{n^{\prime} l^{\prime}, n^{\prime} l^{\prime}}^{x}(r)$, and $\beta_{x} y_{n^{\prime} l^{\prime}, n l}^{x}(r)$, are omitted here and can be found elsewhere [6]. Here, we just note that they define the interaction of the electrons of the $n l$ shell with the other electrons of the same shell, averaged over all angles, and with the electrons of the other shells, including both the usual and the exchange interactions.

In general, the coefficients $f_{x}, \alpha_{x}$, and $\beta_{x}$ depend on the whole set of quantum numbers, defining the atomic level under consideration, and particularly on $L$ and $S$. Consequently, different orbitals (or radial wavefunctions) $\varphi_{n l}, \varphi_{n^{\prime} l^{\prime}}, \ldots$ correspond to different terms of various electron configurations.

In a well-known monograph [8], Cowan introduces the concept of the $L S$-dependent Hartree-Fock ( $L S D$ HF) calculations. His approach uses a different set of radial wavefunctions for each $L S$ term, assuming the pure $L S$-coupling scheme. To be precise, the method should be called $L S v D \mathrm{HF}$, because the total energy for $d$-shells also depends on the seniority number $v$. The number of terms can be large, especially for $d$ - and $f$-shells. Significant simplification can be gained by assuming that for all terms the wavefunctions and eigenvalues are identical and calculated for the center of gravity of the shell. The so-called "average term approximation" works very well for the highly excited states, but is quite unpredictable for the outer and inner shells. In contrast to the average term HF, all LSD HF wavefunctions, eigenfunctions, and Slater integrals depend on the $L$ and $S$ quantum numbers for inner and outer shells. This condition requires longer, but more accurate computations, and very important for calculation the total energies of the atomic levels. Table 3 presents our calculations of the energies of the Xe XI inner shells by the LSD HF method. These are compared to the results obtained by the wellknown Cowan HF average term code, which is widely used in spectroscopic research for identification of atomic levels. The correspondence is within 1-5\%, although our results are consistently lower than those from the Cowan code for the inner shells, and slightly higher for the open shell. Similar results for Sn VIII are presented in Table 4, and the energies for the Sn VIII - Sn XIII, calculated by various methods are shown in Appendix A.

The angular wavefunctions are normally calculated separately to the radial wavefunctions by the summation of the electron momentums. As a rule, the two limited cases are rarely realized in practice, that is, when electrostatic interaction is considered predominant ( $L S$ coupling) or spin-orbit interaction exceeds the electrostatic interaction (jj-coupling). In such intermediate cases, the Hamiltonian matrix cannot be written diagonal in any coupling schemes. Therefore, the complete matrix is written by transforming the Coulomb matrix from $L S$ representation to $j j$-representation. The eigenvalues of the Hamiltonian matrix are found later by numerical diagonalization, and the eigenvector (purity vector) defines the composition of the level, corresponding to this eigenvalue. The level is normally assigned according to the highest 
contribution of the basis term from the purity vector. Note that the energy levels, found within the intermediate coupling, never have 100 percent pure $L S$ - or $j j$-coupling, and the difference always exists between the level assigned within the intermediate coupling or the level calculated within the pure coupling scheme and accordingly assigned as required by the scheme.

The relativistic effects can be negligible for the low- $Z$ elements, but apparently become evident for intermediate- $Z$ and high- $Z$ elements. The most widespread and relatively easy way to account for them within the HF method is by one-electron relativistic corrections within the framework of perturbation theory. The more strict and accurate way to account for relativistic effects is to use the Dirac-Fock approximation, but this significantly complicates the problem, while the gain from it would be pronounced only for the high- $Z$ elements.

Table 3: Energies of Xe XI inner shells by $L S D$ HF and average term HF.

\begin{tabular}{|c||c|c|c|c|c|c|}
\hline \multicolumn{5}{|c||}{} & \multicolumn{5}{c|}{ HEIGHTS-ATOM } & Cowan \\
\cline { 2 - 7 } Conf & ${ }^{1} \mathrm{~S}$ & ${ }^{2} \mathrm{P}$ & ${ }^{1} \mathrm{D}$ & ${ }^{3} \mathrm{~F}$ & ${ }^{1} \mathrm{G}$ & $\mathrm{E}_{\text {av }}$ \\
\hline \hline $1 \mathrm{~s}^{2}$ & 2462.1420 & 2462.1173 & 2462.1165 & 2462.1075 & 2462.1211 & 2565.4407 \\
\hline $2 \mathrm{~s}^{2}$ & 391.7600 & 391.7356 & 391.7349 & 391.7260 & 391.7394 & 417.8558 \\
\hline $2 \mathrm{p}^{6}$ & 365.3100 & 365.2854 & 365.2847 & 365.2757 & 365.2892 & 376.5671 \\
\hline $3 \mathrm{~s}^{2}$ & 92.9495 & 92.9293 & 92.9289 & 92.9216 & 92.9325 & 98.5881 \\
\hline $3 \mathrm{p}^{6}$ & 81.8833 & 81.8629 & 81.8625 & 81.8551 & 81.8661 & 84.8207 \\
\hline $3 \mathrm{~d}^{10}$ & 63.3815 & 63.3609 & 63.3605 & 63.3530 & 63.3642 & 64.1559 \\
\hline $4 \mathrm{~s}^{2}$ & 27.4180 & 27.4061 & 27.4061 & 27.4018 & 27.4080 & 28.6868 \\
\hline $4 \mathrm{p}^{6}$ & 23.1245 & 23.1133 & 23.1132 & 23.1092 & 23.1151 & 23.9933 \\
\hline $4 d^{8}$ & 17.0287 & 17.1508 & 17.1568 & 17.2021 & 17.1329 & 17.0272 \\
\hline
\end{tabular}

Table 4: Energies of Sn VIII inner shells by LSD HF and average term HF.

\begin{tabular}{|c|c|c|c|c|c|c|c|c|c|}
\hline & \multicolumn{8}{|c|}{ HEIGHTS-ATOM } & Cowan \\
\hline Conf & ${ }^{2} \mathbf{P}$ & ${ }^{4} P$ & ${ }_{1}^{2} \mathrm{D}$ & ${ }_{3}^{2} \mathrm{D}$ & ${ }^{2} \mathrm{~F}$ & ${ }^{4} \mathrm{~F}$ & ${ }^{2} \mathbf{G}$ & ${ }^{2} \mathrm{H}$ & $E_{a v}$ \\
\hline $1 \mathrm{~s}^{2}$ & 2091.3064 & 2091.3013 & 2091.3279 & 2091.3127 & 2091.3169 & 2091.2909 & 2091.3029 & 2091.3064 & 2165.7804 \\
\hline $2 s^{2}$ & 322.6554 & 322.6503 & 322.6769 & 322.6617 & 322.6659 & 322.6399 & 322.6520 & 322.6554 & 341.0976 \\
\hline $2 p^{6}$ & 298.6029 & 298.5977 & 298.6244 & 298.6092 & 298.6134 & 298.5873 & 298.5994 & 298.6029 & 307.0733 \\
\hline $3 s^{2}$ & 71.5397 & 71.5351 & 71.5580 & 71.5449 & 71.5484 & 71.5264 & 71.5367 & 71.5397 & 75.3565 \\
\hline $3 p^{6}$ & 61.7069 & 61.7023 & 61.7253 & 61.7121 & 61.7157 & 61.6935 & 61.7039 & 61.7069 & 63.8827 \\
\hline $3 d^{10}$ & 45.3449 & 45.3404 & 45.3633 & 45.3502 & 45.3537 & 45.3315 & 45.3419 & 45.3449 & 46.0725 \\
\hline $4 s^{2}$ & 18.6978 & 18.6948 & 18.7092 & 18.7009 & 18.7030 & 18.6896 & 18.6961 & 18.6978 & 19.4893 \\
\hline $4 p^{6}$ & 15.0560 & 15.0532 & 15.0665 & 15.0589 & 15.0608 & 15.0484 & 15.0544 & 15.0560 & 15.6992 \\
\hline $4 d^{7}$ & 10.2634 & 10.2836 & 10.1689 & 10.2343 & 10.2149 & 10.3322 & 10.2796 & 10.2634 & 10.1565 \\
\hline
\end{tabular}

As follows from Tables 3 and 4, it is very hard to benchmark and verify the accuracy of the computation of such complicated elements as xenon or tin without experimental results. Before proceeding with our tin calculation, we checked the accuracy of our code on well-known elements with available experimental measurements of the levels for oxygen (Table 5) and argon (Table 6). The NIST tables are published in Ref. 20. The simplicity of oxygen and argon comes from the pronounced $L S$-coupling approximation, which allows us to uniquely identify and assign the levels once the intermediate coupling is applied. The average term HF method presents similar, but slightly less accurate results, despite the fact that the average term approximation is expected to work very well for the excited states [8].

As mentioned above, the intermediate- $Z$ elements, such as tin, do not have any pure 
coupling scheme, and the intermediate coupling approximation is the only reasonable choice in computer calculation of atomic levels. However, the assignment of the calculated levels is arbitrary, preserving only the exact quantum number of total momentum $J$. In column 3 of the Table 7, we present our results for the Sn VIII energy levels, and in the column 7 of the same table are the results of the Cowan code. We have ordered the levels by increasing the total moment and arranged the Cowan code levels accordingly to our identification. Since different intermediate coupling codes might have different naming schemes, the reasonable way of presenting the results is arranging the levels accordingly to the total moment of the level. The experimental energy levels are available thanks to the Ref. 23, and we can benchmark the accuracy of these calculations. As one can see, the energy levels computed by us are close to the Cowan code results and differ from the experimentally defined values from $3 \%$ to $12 \%$.

Table 5: O I $2 \mathrm{p}^{3} 3 \mathrm{~s}$ excited state energy levels (in $\mathrm{cm}^{-1}$ ).

\begin{tabular}{|c|c|c|c|c|c|}
\hline \multirow{2}{*}{ Excited level } & \multicolumn{2}{|c|}{ HEIGHTS-ATOM } & \multicolumn{2}{|c|}{ Cowan } & \multirow{2}{*}{$\begin{array}{c}\text { NIST } \\
\text { Tables }\end{array}$} \\
\hline & Level & Acc, $\%$ & Level & Acc & \\
\hline $2 p^{3}\left({ }^{2} P\right) 3 s\left({ }^{2} S\right)^{3} P_{0}$ & 51017.20 & & 56245.60 & & \\
\hline $2 p^{3}\left({ }^{4} S\right) 3 s\left({ }^{2} S\right)^{3} S_{1}$ & 2693.72 & 11.00 & 9454.00 & 212.35 & 3026.78 \\
\hline $2 p^{3}\left({ }^{2} P\right) 3 s\left({ }^{2} S\right)^{1} P_{1}$ & 52458.82 & & 60975.40 & & \\
\hline $2 p^{3}\left({ }^{2} P\right) 3 s\left({ }^{2} s\right)^{3} P_{1}$ & 51017.20 & & 56247.20 & & \\
\hline $2 p^{3}\left({ }^{2} D\right) 3 s\left({ }^{2} S\right)^{3} D_{1}$ & 31117.88 & 13.62 & 34690.20 & 26.67 & 27387.22 \\
\hline $2 p^{3}\left({ }^{4} s\right) 3 s\left({ }^{2} s\right)^{5} S_{2}$ & 0.00 & 0.00 & 0.00 & 0.00 & 0.00 \\
\hline $2 p^{3}\left({ }^{2} P\right) 3 s\left({ }^{2} s\right)^{3} P_{2}$ & 51024.33 & & 56250.60 & & \\
\hline $2 p^{3}\left({ }^{2} D\right) 3 s\left({ }^{2} s\right)^{1} D_{2}$ & 32506.93 & & 39418.50 & & \\
\hline $2 p^{3}\left({ }^{2} D\right) 3 s\left({ }^{2} s\right)^{3} D_{2}$ & 31114.91 & 13.64 & 34691.10 & 26.71 & 27379.33 \\
\hline $2 p^{3}\left({ }^{2} D\right) 3 s\left({ }^{2} s\right)^{3} D_{3}$ & 31117.88 & 13.70 & 34693.20 & 26.77 & 27367.21 \\
\hline
\end{tabular}

Table 6: Ar II 3 $\mathrm{p}^{4}$ 3d excited state energy levels $\left(\right.$ in $\left.\mathrm{cm}^{-1}\right)$.

\begin{tabular}{|c|c|c|c|c|c|}
\hline \multirow{2}{*}{ Excited level } & \multicolumn{2}{|c|}{ HEIGHTS-ATOM } & \multicolumn{2}{|c|}{ Cowan } & \multirow{2}{*}{$\begin{array}{c}\text { NIST } \\
\text { Tables }\end{array}$} \\
\hline & Level & Acc, $\%$ & Level & Acc & \\
\hline $3 \mathrm{p}^{4}\left({ }^{1} \mathrm{D}\right) 3 \mathrm{~d}\left({ }^{2}{ }_{1} \mathrm{D}\right)^{2} \mathrm{~S}_{0.5}$ & 40846.4178 & 21.09 & 52850.1 & 2.1 & 51765.7720 \\
\hline $3 p^{4}\left({ }^{1} D\right) 3 d\left({ }^{2}{ }_{1} D\right)^{2} P_{0.5}$ & 41248.8245 & 2.93 & 18681.8 & 56.0 & 42493.6398 \\
\hline $3 p^{4}\left({ }^{3} P\right) 3 d\left({ }^{2}{ }_{1} D\right)^{2} P_{0.5}$ & 35121.2034 & 183.63 & 93017.9 & 651.2 & 12382.6192 \\
\hline $3 p^{4}\left({ }^{3} P\right) 3 d\left({ }^{2}{ }_{1} D\right)^{4} P_{0.5}$ & 18092.2789 & 21.42 & 22836.6 & 53.3 & 14900.6902 \\
\hline $3 p^{4}\left({ }^{3} P\right) 3 d\left({ }_{1}^{2} D\right)^{4} D_{0.5}$ & 394.0666 & 3.97 & 403.8 & 1.6 & 410.3420 \\
\hline $3 p^{4}\left({ }^{1} S\right) 3 d\left({ }_{1}{ }_{1} D\right)^{2} D_{1.5}$ & 58824.3505 & 23.57 & 63587.7 & 33.6 & 47604.4745 \\
\hline $3 p^{4}\left({ }^{1} D\right) 3 d\left({ }_{1}{ }_{1} D\right)^{2} P_{1.5}$ & 41249.8121 & 1.98 & 19756.3 & 53.1 & 42082.5271 \\
\hline $3 p^{4}\left({ }^{1} D\right) 3 d\left({ }^{2}{ }_{1} D\right)^{2} D_{1.5}$ & 40839.6141 & 0.83 & 24700.9 & 39.0 & 40502.2899 \\
\hline $3 p^{4}\left({ }^{3} P\right) 3 d\left({ }^{2}{ }_{1} D\right)^{2} P_{1.5}$ & 35483.3365 & 165.96 & 92456.2 & 593.0 & 13341.5221 \\
\hline $3 p^{4}\left({ }^{3} P\right) 3 d\left({ }^{2}{ }_{1} D\right)^{4} P_{1.5}$ & 18366.1832 & 21.02 & 23196.9 & 52.9 & 15175.7514 \\
\hline $3 p^{4}\left({ }^{3} P\right) 3 d\left({ }^{2}{ }_{1} D\right)^{2} D_{1.5}$ & 37436.7702 & 106.29 & 103522.9 & 470.4 & 18147.6279 \\
\hline $3 \mathrm{p}^{4}\left({ }^{3} \mathrm{P}\right) 3 \mathrm{~d}\left({ }^{2}{ }_{1} \mathrm{D}\right)^{4} \mathrm{D}_{1.5}$ & 291.3525 & 3.96 & 299.0 & 1.4 & 303.3660 \\
\hline $3 \mathrm{p}^{4}\left({ }^{3} \mathrm{P}\right) 3 \mathrm{~d}\left({ }^{2}{ }_{1} \mathrm{D}\right)^{4} \mathrm{~F}_{1.5}$ & 12253.5961 & 10.95 & 15783.3 & 42.9 & 11044.0744 \\
\hline $3 \mathrm{p}^{4}\left({ }^{1} \mathrm{~S}\right) 3 \mathrm{~d}\left({ }^{2}{ }_{1} \mathrm{D}\right)^{2} \mathrm{D}_{2.5}$ & 58824.2407 & 24.46 & 63853.6 & 35.1 & 47264.8629 \\
\hline $3 p^{4}\left({ }^{1} D\right) 3 d\left({ }^{2}{ }_{1} D\right)^{2} D_{2.5}$ & 41036.0439 & 2.57 & 25648.1 & 35.9 & 40008.2352 \\
\hline $3 p^{4}\left({ }^{1} D\right) 3 d\left({ }^{2}{ }_{1} D\right)^{2} F_{2.5}$ & 37712.7595 & 21.76 & 46206.2 & 49.2 & 30972.1990 \\
\hline $3 p^{4}\left({ }^{3} P\right) 3 d\left({ }^{2}{ }_{1} D\right)^{4} P_{2.5}$ & 18690.6764 & 20.21 & 23679.7 & 52.3 & 15548.5860 \\
\hline $3 \mathrm{p}^{4}\left({ }^{3} \mathrm{P}\right) 3 \mathrm{~d}\left({ }^{2}{ }_{1} \mathrm{D}\right)^{2} \mathrm{D}_{2.5}$ & 36402.7156 & 94.04 & 102099.4 & 444.2 & 18759.9507 \\
\hline $3 p^{4}\left({ }^{3} P\right) 3 d\left({ }^{2}{ }_{1} D\right)^{4} D_{2.5}$ & 110.3957 & 28.24 & 152.4 & 0.9 & 153.8450 \\
\hline $3 p^{4}\left({ }^{3} P\right) 3 d\left({ }^{2}{ }_{1} D\right)^{2} F_{2.5}$ & 22225.5343 & 24.72 & 29109.4 & 63.3 & 17820.3401 \\
\hline $3 \mathrm{p}^{4}\left({ }^{3} \mathrm{P}\right) 3 \mathrm{~d}\left({ }^{2}{ }_{1} \mathrm{D}\right)^{4} \mathrm{~F}_{2.5}$ & 11921.9700 & 10.59 & 15500.2 & 43.8 & 10780.3183 \\
\hline $3 p^{4}\left({ }^{1} D\right) 3 d\left({ }^{2}{ }_{1} D\right)^{2} F_{3.5}$ & 37159.2445 & 19.18 & 46532.6 & 49.2 & 31179.1749 \\
\hline $3 \mathrm{p}^{4}\left({ }^{1} \mathrm{D}\right) 3 \mathrm{~d}\left({ }^{2}{ }_{1} \mathrm{D}\right)^{2} \mathrm{G}_{3.5}$ & 26396.3199 & 20.66 & 29245.5 & 33.7 & 21876.6617 \\
\hline $3 p^{4}\left({ }^{3} P\right) 3 d\left({ }^{2}{ }_{1} D\right)^{4} D_{3.5}$ & 0.0000 & 0.00 & 0.0 & 0.0 & 0.0000 \\
\hline $3 \mathrm{p}^{4}\left({ }^{3} \mathrm{P}\right) 3 \mathrm{~d}\left({ }^{2}{ }_{1} \mathrm{D}\right)^{2} \mathrm{~F}_{3.5}$ & 21387.5803 & 26.92 & 27647.0 & 64.1 & 16851.8825 \\
\hline $3 p^{4}\left({ }^{3} P\right) 3 d\left({ }^{2}{ }_{1} D\right)^{4} F_{3.5}$ & 11623.0456 & 11.87 & 15081.4 & 45.2 & 10389.7346 \\
\hline $3 \mathrm{p}^{4}\left({ }^{1} \mathrm{D}\right) 3 \mathrm{~d}\left({ }^{2}{ }_{1} \mathrm{D}\right)^{2} \mathrm{G}_{4.5}$ & 26396.3199 & 20.66 & 29074.2 & 32.9 & 21876.6617 \\
\hline $3 p^{4}\left({ }^{3} P\right) 3 d\left({ }^{2}{ }_{1} D\right)^{4} F_{3.5}$ & 11120.1196 & 12.79 & 14509.5 & 47.2 & 9858.9536 \\
\hline
\end{tabular}


From a theoretical standpoint, the agreement of the calculated atomic energy levels with the experimentally measured values within $3 \%$ to $12 \%$ may be considered encouraging, but in reality, the required EUV bandwidth has much greater restrictions. The well-known effect of overestimation of the theoretical energy-level splitting results in slightly larger values of Coulomb electron-electron interactions [8, 21]. The electron correlation cannot be determined scaled-down theoretical values of the single-configuration Slater integrals. Any experimental data can actually be a great help in determining the exact values of the scaling factors, as demonstrated in Table 8. The experimental values in the second column are taken from Ref. 22. As expected, the purely theoretical values for the ground energy levels of Xe XI, shown in column 3, are slightly overestimated, by approximately $10 \%$, but scaling the Slater integrals to a factor of 0.82 yields a very accurate result, within less than $2 \%$ for most ground levels. Thanks to the Ref. 22, we can also benchmark the accuracy of our calculation for the Xe XI $4 \mathrm{~d}^{7} 5 \mathrm{p}$ excited levels. Additionally, we have calculated the same energy levels by the Cowan code. The calculated results for Xe XI $4 d^{7} 5 p$ excited levels in Table 9 show excellent agreement with the experimental values, as accurate as $1-2 \%$ for most levels. As evident from the table, the Cowan code produces slightly higher energy values, which would shift the $4 d^{8}-4 d^{7} 5 p$ transition array toward longer wavelengths.

Table 7: Sn VIII $4 d^{7}$ ground state energy levels.

\begin{tabular}{|c|c|c|c|c|c|c|c|}
\hline & \multirow{2}{*}{$\frac{\operatorname{Exp}}{\mathrm{cm}^{-1}}$} & \multicolumn{2}{|c|}{ HEIGHTS-ATOM (th) } & \multicolumn{2}{|c|}{ HEIGHTS-ATOM (fit) } & \multicolumn{2}{|c|}{ Cowan } \\
\hline & & Energy, $\mathrm{cm}^{-1}$ & Acc & Energy, $\mathrm{cm}^{-1}$ & Acc & Energy, $\mathrm{cm}^{-1}$ & Acc \\
\hline $4 d^{72}{ }_{3} P_{0.5}$ & 35458 & 36645 & $3.35 \%$ & 36451 & $2.80 \%$ & 37684 & $6.28 \%$ \\
\hline $4 d^{74}{ }_{3} P_{0.5}$ & 23946 & 24936 & $4.13 \%$ & 23326 & $2.59 \%$ & 25937 & $8.32 \%$ \\
\hline $4 d^{72}{ }_{3} P_{1.5}$ & 30657 & 32889 & $7.28 \%$ & 32137 & $4.83 \%$ & 32347 & $5.51 \%$ \\
\hline $4 d^{74}{ }_{3} P_{1.5}$ & 18280 & 19072 & $4.33 \%$ & 18259 & $0.12 \%$ & 20472 & $11.99 \%$ \\
\hline $4 d^{72}{ }_{1} D_{1.5}$ & - & 69434 & & 70831 & & 75845 & \\
\hline $4 d^{72}{ }_{3} D_{1.5}$ & 44177 & 43707 & $1.06 \%$ & 43468 & $1.60 \%$ & 45637 & $3.31 \%$ \\
\hline $4 d^{74}{ }_{3} F_{1.5}$ & 12153 & 13375 & $10.06 \%$ & 12823 & $5.25 \%$ & 12083 & $0.58 \%$ \\
\hline $4 d^{74}{ }_{3} P_{2.5}$ & 20373 & 21448 & $5.28 \%$ & 19525 & $4.16 \%$ & 22171 & $8.83 \%$ \\
\hline $4 d^{72}{ }_{1} D_{2.5}$ & 75377 & 65418 & $13.21 \%$ & 66819 & $11.35 \%$ & 79115 & $4.96 \%$ \\
\hline $4 d^{72}{ }_{3} D_{2.5}$ & 33670 & 42009 & $24.77 \%$ & 41517 & $23.30 \%$ & 34785 & $3.31 \%$ \\
\hline $4 d^{72}{ }_{3} F_{2.5}$ & 45452 & 45232 & $0.48 \%$ & 44567 & $1.95 \%$ & 48749 & $7.25 \%$ \\
\hline $4 d^{74}{ }_{3} F_{2.5}$ & 10341 & 11157 & $7.89 \%$ & 10605 & $2.49 \%$ & 10120 & $2.13 \%$ \\
\hline $4 d^{72}{ }_{3} F_{3.5}$ & 49476 & 49870 & $0.80 \%$ & 48753 & $1.46 \%$ & 52498 & $6.11 \%$ \\
\hline $4 d^{74}{ }_{3} F_{3.5}$ & 6986 & 7130 & $2.05 \%$ & 6716 & $3.87 \%$ & 6704 & $4.03 \%$ \\
\hline $4 d^{72}{ }_{3} G_{3.5}$ & 29001 & 28378 & $2.15 \%$ & 29338 & $1.16 \%$ & 29358 & $1.23 \%$ \\
\hline $4 d^{74}{ }_{3} F_{4.5}$ & 0 & 0 & & 0 & & 0 & \\
\hline $4 d^{72}{ }_{3} G_{4.5}$ & 22636 & 21884 & $3.32 \%$ & 22976 & $1.50 \%$ & 23082 & $1.97 \%$ \\
\hline $4 d^{72}{ }_{3} H_{4.5}$ & 37751 & 36996 & $2.00 \%$ & 37413 & $0.89 \%$ & 38072 & $0.85 \%$ \\
\hline $4 d^{72}{ }_{3} \mathrm{H}_{5.5}$ & 30312 & 29369 & $3.11 \%$ & 30042 & $0.89 \%$ & 30933 & $2.05 \%$ \\
\hline
\end{tabular}


Table 8: Xe XI $4 \mathrm{~d}^{8}$ ground state energy levels (in $\mathrm{cm}^{-1}$ ).

\begin{tabular}{|c||c||c|c||c|c|}
\hline & Exp & Theor & Acc, $\%$ & Modified & Acc, $\%$ \\
\hline \hline $4 d^{83} F_{4}$ & 0 & 0 & & 0 & \\
\hline $4 d^{83} F_{3}$ & 13140 & 15069 & 14.68 & 13184 & 0.34 \\
\hline $4 d^{83} F_{2}$ & 15205 & 15456 & 1.65 & 15219 & 0.09 \\
\hline $4 d^{83} P_{2}$ & 26670 & 29402 & 10.24 & 27025 & 1.33 \\
\hline $4 d^{83} P_{0}$ & 32210 & 37699 & 17.04 & 32410 & 0.62 \\
\hline $4 d^{83} P_{1}$ & 34610 & 39394 & 13.82 & 35145 & 1.54 \\
\hline $4 d^{8} G_{4}$ & 40835 & 45551 & 11.55 & 39917 & 2.25 \\
\hline $4 d^{8} D_{2}$ & 42900 & 46204 & 7.7 & 43006 & 0.25 \\
\hline $4 d^{8} S_{0}$ & 88130 & 99330 & 12.71 & 85587 & 2.89 \\
\hline
\end{tabular}

Table 9: Xe XI $4 d^{7} 5 p$ excited state energy levels (in $\mathrm{cm}^{-1}$ ).

\begin{tabular}{|c|c|c|c|c|c|c|c|c|c|c|c|c|c|}
\hline J & HEIGHTS & Exp & Acc, $\%$ & Cowan & Exp & Acc, $\%$ & $\mathbf{J}$ & HEIGHTS & Exp & Acc, $\%$ & Cowan & Exp & Acc, $\%$ \\
\hline \multirow{7}{*}{ 음 } & 753682 & & & 704730 & & & \multirow{20}{*}{$\begin{array}{l}m \\
\text { II }\end{array}$} & 748359 & 739322 & 1.21 & 717807 & 733755 & 2.22 \\
\hline & 764819 & & & 724612 & & & & 752695 & 741800 & 1.45 & 720466 & 739322 & 2.62 \\
\hline & 767530 & & & 727272 & & & & 758000 & 744955 & 1.72 & 723565 & 741800 & 2.52 \\
\hline & 776382 & & & 737419 & & & & 763470 & 746445 & 2.23 & 724781 & 744955 & 2.78 \\
\hline & 789881 & 792311 & 0.31 & 757151 & & & & 771677 & 749351 & 2.89 & 728734 & 746445 & 2.43 \\
\hline & 796489 & & & 758364 & & & & 773934 & 752054 & 2.83 & 731322 & 749351 & 2.47 \\
\hline & 835707 & & & 771196 & 792311 & 2.74 & & 774880 & 754860 & 2.58 & 733156 & 752054 & 2.58 \\
\hline \multirow{19}{*}{ i⿱ } & 542235 & 742594 & 36.95 & 693853 & & & & 777965 & 759110 & 2.42 & 738201 & 754860 & 2.26 \\
\hline & 757317 & 745470 & 1.56 & 702217 & & & & 781014 & 761266 & 2.53 & 739969 & 759110 & 2.59 \\
\hline & 765462 & 752155 & 1.74 & 710994 & & & & 785056 & 766860 & 2.32 & 745423 & 761266 & 2.13 \\
\hline & 771635 & 754745 & 2.19 & 722192 & & & & 790166 & 768773 & 2.71 & 747692 & 766860 & 2.56 \\
\hline & 777881 & 758337 & 2.51 & 724441 & 742594 & 2.51 & & 792063 & 773715 & 2.32 & 752379 & 768773 & 2.18 \\
\hline & 779903 & 760950 & 2.43 & 730621 & 745470 & 2.03 & & 792759 & 775570 & 2.17 & 754544 & 773715 & 2.54 \\
\hline & 781248 & 765770 & 1.98 & 735493 & 752155 & 2.27 & & 797858 & 780503 & 2.18 & 759955 & 775570 & 2.05 \\
\hline & 785224 & 767369 & 2.27 & 738123 & 754745 & 2.25 & & 801299 & 788465 & 1.60 & 767977 & 780503 & 1.63 \\
\hline & 788088 & 775030 & 1.66 & 739352 & 758337 & 2.57 & & 810423 & 795135 & 1.89 & 774072 & 788465 & 1.86 \\
\hline & 792118 & 778350 & 1.74 & 745760 & 760950 & 2.04 & & 813769 & 801225 & 1.54 & 781651 & 795135 & 1.73 \\
\hline & 793228 & 784035 & 1.16 & 746488 & 765770 & 2.58 & & 826715 & 824474 & 0.27 & 789720 & 801225 & 1.46 \\
\hline & 795405 & 788396 & 0.88 & 754717 & 767369 & 1.68 & & 830391 & 838289 & 0.95 & 804178 & 824474 & 2.52 \\
\hline & 802074 & 791805 & 1.28 & 763654 & 775030 & 1.49 & & 859482 & & & 818639 & 838289 & 2.40 \\
\hline & 807341 & 808130 & 0.10 & 768242 & 778350 & 1.32 & \multirow{18}{*}{$\begin{array}{l}\nabla \\
\text { II }\end{array}$} & 720948 & 695376 & 3.55 & 674946 & & \\
\hline & 812504 & & & 771542 & 784035 & 1.62 & & 729055 & 712223 & 2.31 & 691130 & 695376 & 0.61 \\
\hline & 821941 & & & 788076 & 788396 & 0.04 & & 733795 & 725053 & 1.19 & 703565 & & \\
\hline & 841501 & 830260 & 1.34 & 798662 & 791805 & 0.86 & & 737916 & 731458 & 0.88 & 709989 & & \\
\hline & 843731 & & & 801863 & 808130 & 0.78 & & 740340 & 737388 & 0.40 & 714976 & 712223 & 0.38 \\
\hline & 846356 & & & 810811 & 830260 & 2.40 & & 757762 & 739542 & 2.40 & 718830 & & \\
\hline \multirow{25}{*}{ N } & 691417 & & & 687857 & & & & 761448 & 744537 & 2.22 & 721907 & 725053 & 0.44 \\
\hline & 724852 & 715730 & 1.26 & 692334 & & & & 763104 & 752285 & 1.42 & 731022 & 731458 & 0.06 \\
\hline & 728526 & 721001 & 1.03 & 696514 & & & & 766332 & 755831 & 1.37 & 733091 & 737388 & 0.59 \\
\hline & 732116 & 740757 & 1.18 & 700235 & & & & 773027 & 756016 & 2.20 & 734544 & 739542 & 0.68 \\
\hline & 746236 & 746552 & 0.04 & 717679 & 715730 & 0.27 & & 775476 & 763070 & 1.60 & 741653 & 744537 & 0.39 \\
\hline & 749293 & 750512 & 0.16 & 718894 & & & & 780864 & 773968 & 0.88 & 750301 & 752285 & 0.26 \\
\hline & 753451 & 753795 & 0.05 & 725032 & 721001 & 0.56 & & 786082 & 775775 & 1.31 & 752696 & 755831 & 0.42 \\
\hline & 758428 & 756170 & 0.30 & 726551 & & & & 799561 & & & 754242 & 756016 & 0.24 \\
\hline & 761149 & 762105 & 0.13 & 729295 & & & & 801886 & & & 761942 & 763070 & 0.15 \\
\hline & 767927 & 765052 & 0.37 & 732909 & & & & 804653 & & & 769559 & 773968 & 0.57 \\
\hline & 769318 & 766625 & 0.35 & 735891 & 740757 & 0.66 & & 826400 & & & 775551 & 775775 & 0.03 \\
\hline & 773092 & 773315 & 0.03 & 738595 & 746552 & 1.08 & & 830536 & & & 805690 & & \\
\hline & 779656 & & & 741586 & 750512 & 1.20 & \multirow{11}{*}{$\begin{array}{l}\text { in } \\
\text { II } \\
7\end{array}$} & 683225 & 722439 & 5.74 & 677821 & & \\
\hline & 780705 & 781822 & 0.14 & 744032 & 753795 & 1.31 & & 732407 & 730345 & 0.28 & 699866 & 722439 & 3.23 \\
\hline & 783294 & & & 746840 & 756170 & 1.25 & & 739736 & 738248 & 0.20 & 709556 & 730345 & 2.93 \\
\hline & 786437 & 786580 & 0.02 & 752933 & 762105 & 1.22 & & 746958 & 740348 & 0.88 & 714977 & 738248 & 3.25 \\
\hline & 789616 & 788145 & 0.19 & 755918 & 765052 & 1.21 & & 763685 & 753352 & 1.35 & 719344 & 740348 & 2.92 \\
\hline & 790623 & & & 760587 & 766625 & 0.79 & & 769708 & 759260 & 1.36 & 731954 & 753352 & 2.92 \\
\hline & 793917 & 795995 & 0.26 & 764895 & 773315 & 1.10 & & 771325 & 766947 & 0.57 & 737152 & 759260 & 3.00 \\
\hline & 798349 & & & 767897 & 781822 & 1.81 & & 773197 & 767700 & 0.71 & 744858 & 766947 & 2.97 \\
\hline & 804334 & 802905 & 0.18 & 774832 & 786580 & 1.52 & & 785525 & 773968 & 1.47 & 751356 & 767700 & 2.18 \\
\hline & 809562 & & & 782382 & 788145 & 0.74 & & 814311 & 789029 & 3.10 & 766642 & 773968 & 0.96 \\
\hline & 815205 & 828875 & 1.68 & 783192 & 795995 & 1.63 & & 823737 & & & 775264 & 789029 & 1.78 \\
\hline & 868819 & & & 808808 & 802905 & 0.73 & \multirow{5}{*}{$\begin{array}{l}0 \\
\| I \\
\longrightarrow\end{array}$} & 730685 & & & 700419 & & \\
\hline & 981088 & & & 815096 & 828875 & 1.69 & & 765587 & & & 719093 & & \\
\hline \multirow{4}{*}{$\begin{array}{l}m \\
\text { II } \\
\longrightarrow\end{array}$} & 723800 & 709285 & 2.01 & 694244 & & & & 768696 & & & 731718 & & \\
\hline & 730113 & 714855 & 2.09 & 699416 & 709285 & 1.41 & & 774523 & & & 749845 & & \\
\hline & 736320 & 725825 & 1.43 & 704967 & 714855 & 1.40 & & 782724 & & & 755705 & & \\
\hline & 743547 & 733755 & 1.32 & 712164 & 725825 & 1.92 & 7 & 775010 & & & 739827 & & \\
\hline
\end{tabular}


The results, presented in Table 7 above were calculated in two steps. In the first step, we obtained the total energies of the ion by a pure analytical method. Results of this calculation are presented in column 3, and their relative accuracy compared to the measurements in Ref. 23 is shown in column 4. As expected, the pure theoretical method does not allow us to obtain an accuracy of $2 \%$ for the atomic levels of such a complicated ion as Sn VIII. We have shaded those levels for which the calculations are not accurate enough. In the second step, we used the experimentally defined values and found that at $F_{f}^{2}(d d)=0.82 \cdot F_{t}^{2}(d d), F_{f}^{4}(d d)=$ $0.91 \cdot F_{t}^{4}(d d)$, and $\zeta_{f}(d)=0.96 \cdot \zeta_{t}(d)$ our values agree noticeably better, as shown by columns 5 and 6 in Table 7. In the formulas above, the subscript $f$ means fit value, and the subscript $t$ means theoretical value. At last, we have calculated the same levels by the Cowan code, which despite being purely theoretical, is still utilizing scaling factors near $10 \%$, depending upon the nuclear charge of the element [8]. Our calculations for the excited Sn VIII $4 d^{6} 5 p^{1}$ energy levels are presented in Appendix B. Experimental values are taken from Ref. 23, and the fit values were determined as $\quad F_{f}^{2}(d d)=0.57 \cdot F_{t}^{2}(d d), \quad F_{f}^{4}(d d)=2.11 \cdot F_{t}^{4}(d d), \quad \zeta_{f}(d)=0.44 \cdot \zeta_{t}(d)$, $\zeta_{f}(p)=0.35 \cdot \zeta_{t}(p), F_{f}^{2}(p d)=0.76 \cdot F_{t}^{2}(p d)$, and $G_{f}^{3}(p d)=1.14 \cdot G_{t}^{3}(p d)$. As before, the theoretical values agreed with the experimental data within $5 \%$, and once the experimental values are taken into account, the accuracy of our numerical simulation becomes less than $1 \%$ in most cases.

In sum, our calculated results compare favorably with available experimental data and a Cowan-average-term Hartree-Fock code, as well as other atomic codes with relativistic corrections. At the same time, the accuracy of the purely theoretical methods is not within the required $2 \%$, so without the experimental values, one cannot guarantee the calculation of accurate EUV transitions. We have attained an accuracy within 3-7\% of our purely theoretical calculations for Sn ions which lack experimental energy values. Once the energy levels are obtained, we can significantly improve our calculations to practically as accurate as the measured data.

\section{DETAILED TIN EUV TRANSITIONS}

Emission of light takes place when an atom changes from a state of higher energy to a state of lower energy. Similarly, absorption results in an upward transition that is caused by the action of the radiation field on an atom. The energy of a transition from state $i$ to state $j$ is formally defined as the difference of total energies of the atom or the ion in states $i$ and $j$. As well known from the literature [6 - 8], the following are equivalent measures of total strength of the spectrum line: the probability of a transition $W_{i j}$, the radiation intensity of a spectrum line $I_{i j}$, and the weighted spontaneous-emission transition probability $g_{j} A_{j i}$, expressed through the statistical weight of the level $g_{j}=2 J+1$ and the Einstein spontaneous emission transition probability rate $A_{j i}$. In our study, we normally use the non-dimensional (absorption) oscillator strength, which is related to the line strength $S$ by the following expression: 


$$
f_{i j}=\frac{1}{3} \frac{\left(E_{j}-E_{i}\right)}{2 J+1} S .
$$

As above, unless otherwise stated, we use atomic units. The energy of the transition $\left(E_{j}-E_{i}\right)$ is given in Rydberg units, $2 J+1$ is the degeneracy of the initial level, and the line strength is defined by the matrix element of the wavefunctions of the initial and final states with the electric dipole operator.

Using as before the intermediate coupling approximation, one can determine the wavefunctions of both states in the form of a linear combination of $L S$-coupled basic functions, where the coefficients of the components are obtained from the energy eigenvectors for the correspondent states. This allows us to represent the line strength in the form of the two dot products of the two component vectors to the dipole-transition matrix. Such a technique reduces the problem of calculating the line-strength matrix elements with uncoupled states to the matrix elements with the $L S$-coupled basis functions. The mathematical expressions for the matrix elements are rather complex and lengthy, so we direct the reader to the more complete theoretical publications $[7,24,25]$. Using traditional atomic theory notation for $6 \mathrm{j}-$ and $9 \mathrm{j}$-symbols, fractional parentage coefficients, and square brackets, we only present the final expressions for calculation of the line strengths of typical transitions:

$$
\begin{aligned}
& D_{L S}\left(l_{1}^{n} l_{2}^{k-1}-l_{1}^{n-1} l_{2}^{k}\right)=\delta_{S S^{\prime}}(-1)^{k+l_{2}+L_{2}+L_{2}^{\prime}+L+S_{1}^{\prime}+S_{2}^{\prime}-J^{\prime}}\left(n k\left[L_{1}, S_{1}, L_{2}^{\prime}, S_{2}^{\prime}, L, L^{\prime}, J, J^{\prime}\right]\right)^{0.5} \times \\
& \times\left\{\begin{array}{lll}
L & S & J \\
J^{\prime} & 1 & L^{\prime}
\end{array}\right\}\left\{\begin{array}{ccc}
S_{1}^{\prime} & S_{2}^{\prime} & S \\
S_{2} & S_{1} & s
\end{array}\right\}\left\{\begin{array}{ccc}
L_{1} & L_{1}^{\prime} & l_{1} \\
L_{2} & L_{2}^{\prime} & l_{2} \\
L & L^{\prime} & 1
\end{array}\right\} \times \\
& \times\left(l _ { 1 } ^ { n } \alpha _ { 1 } L _ { 1 } S _ { 1 } \{ l _ { 1 } ^ { n - 1 } \alpha _ { 1 } ^ { \prime } L _ { 1 } ^ { \prime } S _ { 1 } ^ { \prime } ) \left(l_{2}^{k-1} \alpha_{2} L_{2} S_{2}\left\{l_{2}^{k} \alpha_{2}^{\prime} L_{2}^{\prime} S_{2}^{\prime}\right) P_{l_{1} 2}^{(1)},\right.\right. \\
& \begin{aligned}
D_{L S}\left(l_{1}^{n}-l_{1}^{n-1} l_{2}\right) & =\delta_{S_{S} S^{\prime}}(-1)^{L_{1}^{\prime}+l_{2}+S_{1}+J^{\prime}}\left(n\left[L_{1}, L^{\prime}, J, J^{\prime}\right]\right)^{0.5} \times \\
& \times\left\{\begin{array}{ccc}
L_{1} & S & J \\
J^{\prime} & 1 & L^{\prime}
\end{array}\right\}\left\{\begin{array}{ccc}
l_{1} & L_{1}^{\prime} & L_{1} \\
L^{\prime} & 1 & l_{2}
\end{array}\right\}\left(l_{1}^{n} \alpha_{1} L_{1} S_{1}\left\{l_{1}^{n-1} \alpha_{1}^{\prime} L_{1}^{\prime} S_{1}^{\prime}\right) P_{l_{1} l_{2}}^{(1)} .\right.
\end{aligned}
\end{aligned}
$$

In this study, we do not have more than two open shells, the acute symbol is used for the core quantum numbers, the $\delta$-function expresses the selection rule of nonchangeable spin momentum, and the radial integral $P_{l_{1} l_{2}}^{(1)}$ is defined by the matrix element of the radial wavefunctions of participating shells:

$$
P_{l_{l} l_{2}}^{(1)}=\delta_{l_{2}, l_{1} \pm 1}(-1)^{l_{1}+\max \left(l_{1}, l_{2}\right)} \sqrt{\max \left(l_{1}, l_{2}\right)} \int_{0}^{\infty} P_{n_{l_{1}}} r P_{n_{2} l_{2}} d r .
$$

Electric dipole transitions can occur only when the oscillator strength (and, correspondently, the line strength) in (7.1) is non-zero. From the properties of the matrix element in the definition of the line strength $S$, it follows that a transition can occur only when the participating states have opposite parity, and $\Delta J=J-J^{\prime}=0, \pm 1$. The transition $J=J^{\prime}=0$ is not allowed.

In calculating the EUV radiation from tin or xenon plasma, another difficulty may take 
place, such as accounting for the transitions from the inner shells. As reported in recent studies [4], some inner levels of the ions with intermediate and heavy atomic numbers may have energies comparable to the ionization potential, which significantly decreases the accuracy of the HF method. For example, in xenon or tin plasma with major radiating wavelength region of interest near $13 \mathrm{~nm}$, transitions from outer shells $4 d^{q}-4 d^{q-1} 5 p$ must be considered in combination with the transitions $4 d^{q}-4 d^{q-1} 4 f$ and the transitions from the inner shells, such as $4 p^{6} 4 d^{q}-4 p^{5} 4 d^{q+1}$.

The existence of semi-empirical or experimental information on atomic energy levels may greatly help in improving the accuracy of the ab initio simulation. Unfortunately, such information for the intermediate and high- $Z$ elements, especially their highly excited ions, is not fully presented in the literature [26]. For example, no Sn experimental data are available for the EUV region, except the work of Azarov and Joshi [23], which is not actually dedicated to the $13.5 \mathrm{~nm}$ range and only deals with the ion Sn VIII and its transition $4 d^{7}-4 d^{6} 5 p^{1}$. According to them, the transition has a very wide range of splitting (near $6 \mathrm{~nm}$ ), with the major lines being concentrated around 22-23 nm (left figure in Fig. 5). As shown by the right figure in Fig. 5, results of our calculation show very good agreement in shape, width, and place of the transition.

Figure 6 presents the results of our calculation of the EUV transitions for six tin ions, from Sn VIII to Sn XIII. Details of these calculations are presented in Appendix C, and the summary is shown in Table 10.

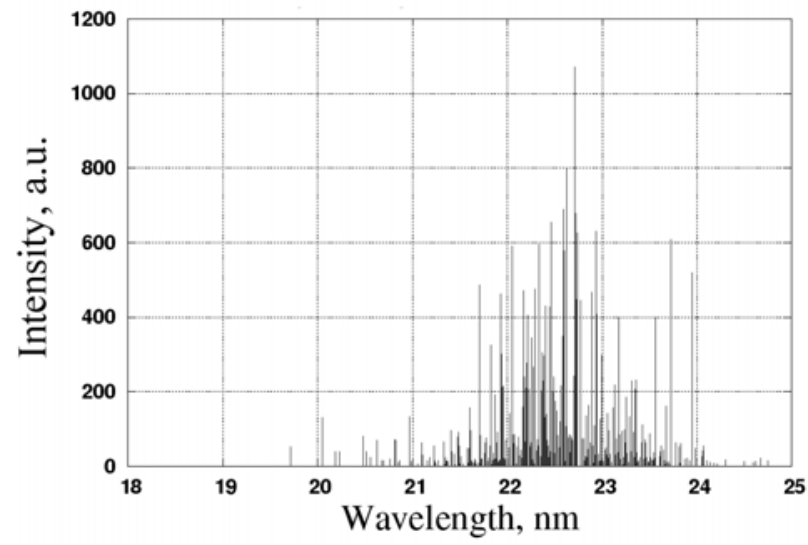

V.I. Azarov and Y.N. Yoshi [23]

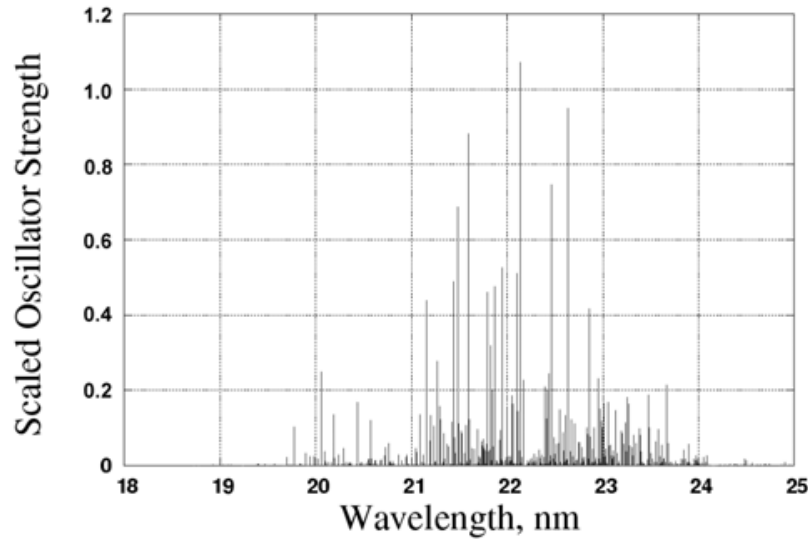

HEIGHTS-ATOM calculation

Fig. 5: Experimental data vs. calculation for Sn VIII $4 d^{7}-4 d^{6} 5 p^{1}$ transition

We have determined that the $4 d-4 f$ and $4 d-5 p$ transitions only partly cover the EUV range of interest. Among the five ions starting from Sn IX, the highest EUV emission should be from the Sn XI $4 d^{4}-4 d^{3} 4 f$ transition and from the Sn XII $4 d^{3}-4 d^{2} 5 p$ transition. Similar results were obtained by means of the Cowan code and are presented in Fig. 7. Despite the difference in the highest values of the oscillator strength, the code also predicts the partial coverage of the EUV range by several transitions, when the highest emission corresponds to the Sn X $4 d^{5}-4 d^{4} 4 f$ and Sn XI $4 d^{4}-4 d^{3} 4 f$ transitions. 

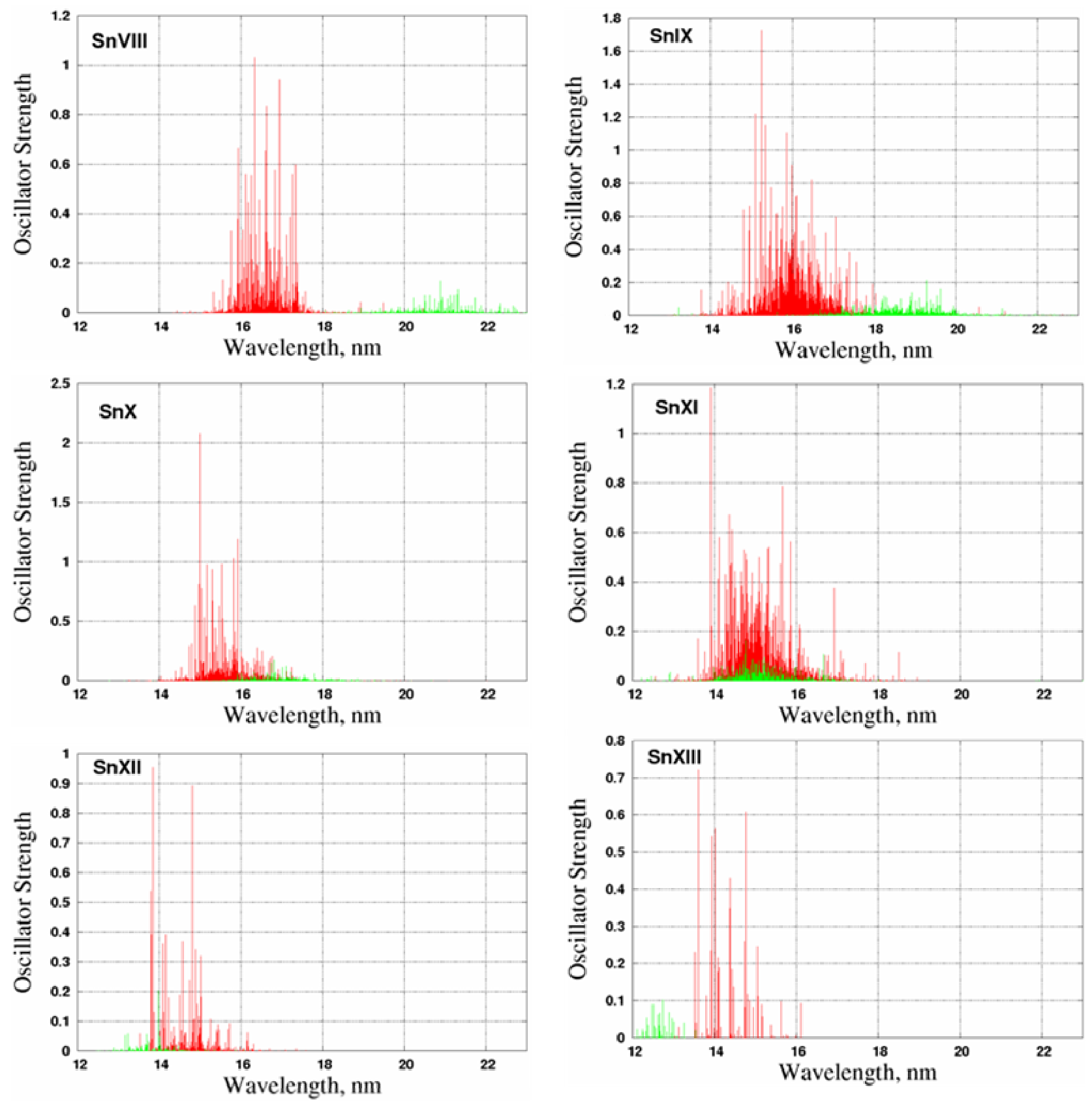

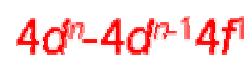

$4 d^{n}-4 d^{n-1} 5 p^{1}$

Fig. 6: Calculation of tin EUV transitions by the HEIGHTS-ATOM code 

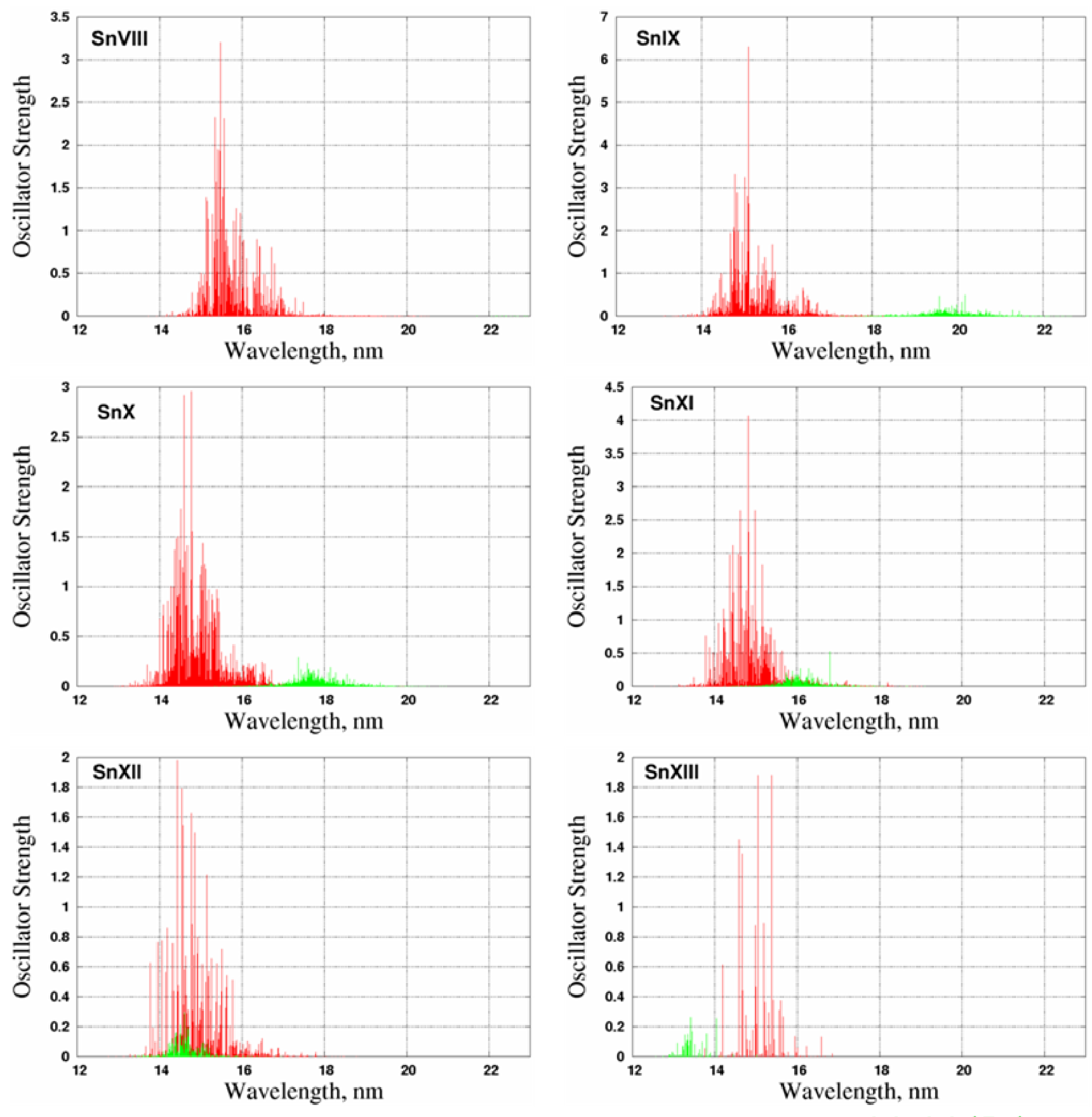

Fig. 7: Calculation of tin EUV transitions by the Cowan code 
Table 10: Summary of the tin EUV transitions.

\begin{tabular}{|l|c|c|c|c|c|c|}
\hline & Sn VIII & Sn IX & Sn X & Sn XI & Sn XII & Sn XIII \\
\hline Ground Configuration & $4 d^{\prime}$ & $4 d^{6}$ & $4 d^{5}$ & $4 d^{4}$ & $4 d^{3}$ & $4 d^{2}$ \\
\hline \# levels in GC & 19 & 34 & 37 & 34 & 19 & 9 \\
\hline Excited 4f Configuration & $4 d^{6} 4 f^{\prime}$ & $4 d^{5} 4 f^{1}$ & $4 d^{4} 4 f^{1}$ & $4 d^{3} 4 f^{1}$ & $4 d^{2} 4 f^{1}$ & $4 d^{1} 4 f^{1}$ \\
\hline \# levels in 4f EC & 346 & 416 & 346 & 206 & 81 & 20 \\
\hline Transition array & $4 d^{\prime}-4 d^{6} 4 f^{1}$ & $4 d^{6}-4 d^{5} 4 f^{1}$ & $4 d^{5}-4 d^{4} 4 f^{1}$ & $4 d^{4}-4 d^{3} 4 f^{1}$ & $4 d^{3}-4 d^{2} 4 f^{1}$ & $4 d^{2}-4 d^{1} 4 f^{1}$ \\
\hline Total oscillator strength & 6.89 & 5.89 & 4.80 & 3.72 & 2.69 & 1.72 \\
\hline Splitting, nm & $14.204-22.869$ & $12.400-23.859$ & $12.501-22.036$ & $11.963-20.205$ & $12.770-18.238$ & $13.029-16.116$ \\
\hline Total transitions & 2326 & 4521 & 4527 & 2391 & 590 & 62 \\
\hline EUV transitions & 0 & 15 & 63 & 101 & 41 & 5 \\
\hline Excited 5p Configuration & $4 d^{6} 5 p^{1}$ & $4 d^{5} 5 p^{1}$ & $4 d^{4} 5 p^{1}$ & $4 d^{3} 5 p^{1}$ & $4 d^{2} 5 p^{1}$ & $4 d^{1} 5 p^{1}$ \\
\hline \# levels in 5p EC & 180 & 214 & 180 & 110 & 45 & 12 \\
\hline Transition array & $4 d^{1}-4 d^{6} 5 p^{1}$ & $4 d^{6}-4 d^{5} 5 p^{1}$ & $4 d^{5}-4 d^{4} 5 p^{1}$ & $4 d^{4}-4 d^{3} 5 p^{1}$ & $4 d^{3}-4 d^{2} 5 p^{1}$ & $4 d^{2}-4 d^{1} 5 p^{1}$ \\
\hline Total oscillator strength & 0.80 & 0.69 & 0.58 & 0.46 & 0.35 & 0.23 \\
\hline Splitting, nm & $15.580-27.152$ & $12.506-46.903$ & $11.661-22.335$ & $11.644-25.673$ & $11.675-16.077$ & $11.915-13.527$ \\
\hline Total transitions & 1456 & 2971 & 2759 & 1571 & 388 & 46 \\
\hline EUV transitions & 0 & 0 & 2 & 66 & 104 & 3 \\
\hline
\end{tabular}

\section{DETAILED TIN EUV OPACITIES}

As discussed in Section 4, the optical emission and absorption coefficients are calculated within the CRE model with line splitting. This model is based on the Hartree-Fock-Slater (HFS) method with both electrostatic and spin-orbit splitting of configurations and spectral lines. Instead of the Racah theory of angular moments for splitting the HFS configuration average energies into levels, we use the better approximation for the split energy levels instead, obtained by the relaxed core Hartree-Fock method in intermediate coupling. In this way, the HFS data for the EUV ions, configurations and transitions are removed, and substituted the improved data directly in the CRE. This guarantees that the EUV range will be covered only by those transitions that we have calculated accurately by the HF method, while the other energy ranges will still be calculated as previously. The size of the generated EUV optical tables is not very large (around 20-40 MB of disk space) due to the very narrow energy range and relatively small number of transitions within that range. Therefore, it is unnecessary to provide additional modifications of the result tables, such as group averaging, as we did previously to reduce the number of spectral points and the amount of required disk space.

In Fig. 8 we present results of our computation of the Sn emission and absorption coefficients in the EUV range of 13.23-13.77 $\mathrm{nm}$ or $90.04-93.71 \mathrm{eV}$, for the typical EUV temperature and density ranges, such as $10-30 \mathrm{eV}$ and $10^{16}-10^{19} \mathrm{~cm}^{-3}$. The coefficients are very dense, when density is around $10^{17} \mathrm{~cm}^{-3}$, and become sparse when the density is higher (up to $10^{19} \mathrm{~cm}^{-3}$ ) or lower (below $10^{16} \mathrm{~cm}^{-3}$ ). The results of this calculation indicate that, to maximize the EUV output, the EUV source needs to operate within the named density range. 

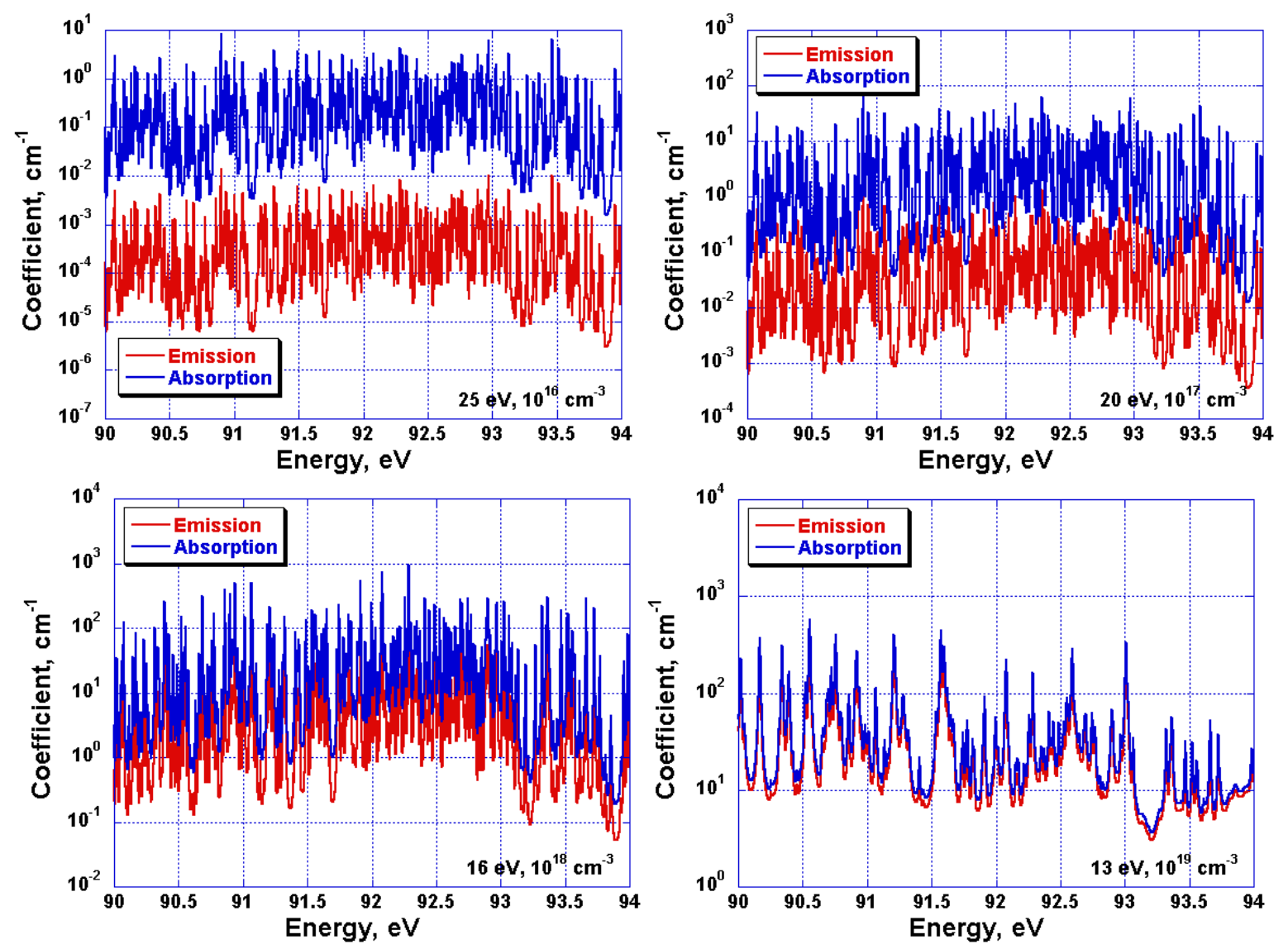

Fig. 8: Tin plasma optical coefficients in the EUV range

\section{CONCLUSION}

The report reviewed the major atomic and plasma methods we use within the comprehensive HEIGHTS-EUV package. The methods differ in accuracy, completeness, and complication. The ion states, populations of atomic levels, and optical coefficients, such as emission and absorption coefficients, are calculated by means of the combination of the HFS atomic model and CRE plasma model with splitting atomic levels. The accuracy of these methods is satisfactory to simulate the plasma magnetohydrodynamic behavior, but insufficient to generate the plasma spectroscopic characteristics. Significant accuracy improvement is achieved by using the advanced LSD HF in intermediate coupling atomic method. However, empirical correction of atomic energy levels is necessary to obtain the spectroscopic accuracy of the data and meet the $2 \%$ bandwidth requirement of leading EUV source manufacturers within the $13.5 \mathrm{~nm}$ range. The applicability of the methods is demonstrated for the tin ions and plasma. Detail atomic and plasma properties, such as energy levels, oscillator strengths, equation of state, relative ion concentrations, wide range opacities, and EUV range opacities near $13.5 \mathrm{~nm}$ are calculated, and compared with the available experimental results. The presented results are used by the authors in simulation the dynamics and characteristics of various EUV sources. 


\section{ACKNOWLEDGMENTS}

The major part of this work is supported by the International SEMATECH and Intel Corp.

\section{REFERENCES}

1. Peter J. Silverman, “The Intel Lithography Roadmap,” Intel Technology Journal, 6 (2), 55 (2002).

2. V. Bakshi, "Welcome and Introduction," EUV Source Workshop, Santa Clara, CA, Feb. 22, 2004.

3. A. Hassanein, V. Sizyuk, V. Tolkach, V. Morozov, and B. Rice, "HEIGHTS Initial Simulation of Discharge Produced Plasma Hydrodynamics and Radiation Transport for EUV Lithography," presented at the 28th SPIE Annual International Symposium and Education Program on Microlithography, Santa Clara, CA, February 23-28, 2003, Proc. of SPIE, Vol. 5037, 714-727.

4. A. Hassanein, V. Sizyuk, V. Tolkach, V. Morozov, T. Sizyuk, B. Rice, and V. Bakshi, "Simulation and Optimization of DPP Hydrodynamics and Radiation Transport for EUV Lithography Devices," Proc. of SPIE Microlithography 2004 Conference, Santa Clara, CA, Feb. 22-27, 2004, pp. 413-422.

5. V. Tolkach, V. Morozov, and A. Hassanein, "Development of Comprehensive Models for Opacities and Radiation Transport for IFE Systems," ANL Report ANL-ET/02-23 (2002).

6. I. I. Sobelman, Introduction to the Theory of Atomic Spectra, Oxford, Pergamon Press (1972).

7. E. U. Condon and G. H. Shortly, The Theory of Atomic Spectra, U.K., Cambridge University Press (1964).

8. R. D. Cowan, The Theory of Atomic Structure and Spectra, Berkeley, Univ. of California Press (1981).

9. J. C. Slater, Phys. Rev. 81, 385 (1951).

10. G. Racah, Phys. Rev. 76, 1352 (1949).

11. G. Racah, Phys. Rev. 85, 381 (1952).

12. T. Holstein, Phys. Rev. 72, 1212 (1947).

13. J. P. Apruzese, et al., J. Quant. Spectrosc. Radiat. Transfer, 23, 479 (1980).

14. J. Pankert et al, Status of Philips Extreme UV Source, unpublished notes.

15. N.N. Kalitkin, Properties of the Matter and MHD-Programs, Preprint, M.V. Keldysh Institute of Applied Mathematics, Moscow, 85, 1985 (in Russian). 
16. L. Huxley and R. Crompton, The Diffusion and Drift of Electrons in Gases, Woodhouse, NY, Wiley (1974).

17. H. R. Griem, Spectral Line Broadening by Plasmas, Academic Press, New York (1974).

18. H. Würz, S. Pestchanyi, I. Landman, B. Bazylev, V. Tolkach, and F. Kappler, Fusion Sci. Technol. 40, N3, 191 (2001).

19. B. N. Bazylev, V. I. Tolkach, et al., "The Calculation of Optical Properties of Atoms and Ions," Kernforschungzentrum Karlsruhe, Interner Bericht (1994).

20. http://physics. nist.gov.

21. R. D. Cowan and N. J. Peacock, Astrophys. J. 142, 390 (1965).

22. S.S. Churiliov, Y.N. Yoshi, J. Reader, and R.R. Kildiyarova, "Analysis of the $4 p^{6} 4 d^{8}-$ $\left(4 d^{7} 5 p+4 d^{7} 4 f+4 p^{5} 4 d^{9}\right)$ Transitions in Xe XI Ion", to be published.

23. V. I. Azarov and Y. N. Joshi, "Analysis of the $4 d^{7}-4 d^{6} 5 p$ Transition Array of the Eighth Spectrum of Tin: Sn VIII,” J. Phys. B: At. Mol. Opt. Phys. 26, 3495-3514 (1993).

24. U. Fano and G. Racah, Irreducible Tensorial Sets, Academic Press, New York (1959).

25. G. Racah, II, Phys. Rev. 62, 438 (1942).

26. Ch. E. Moore, "Atomic Energy Levels as Derived from the Analyses of Optical Spectra," U.S. Dept. of Commerce, National Bureau of Standards, Washington, D.C., p.3 (1949). 


\section{APPENDIX A}

The tables that follow present the Sn VIII - Sn XIII energies of the inner shells, calculated by the HEIGHTS-ATOM relaxed-core LSD HF (for each ground term), and configuration-average energies, calculated by the HEIGHTS-ATOM HFS (HFS), and the Cowan code (CHF). All energies are given in Ry.

Table A 1: Energies of Sn VIII inner shells by various methods.

\begin{tabular}{|c||c|c|c|c|c|c|c|c|c|}
\hline & $1 \mathrm{~s}^{2}$ & $2 \mathrm{~s}^{2}$ & $2 \mathrm{p}^{6}$ & $3 \mathrm{~s}^{2}$ & $3 \mathrm{p}^{6}$ & $3 \mathrm{~d}^{10}$ & $4 \mathrm{~s}^{2}$ & $4 \mathrm{p}^{6}$ & $4 \mathrm{~d}^{7}$ \\
\hline \hline${ }_{3} \mathrm{P}$ & 2091.31 & 322.66 & 298.60 & 71.54 & 61.71 & 45.34 & 18.70 & 15.06 & 10.26 \\
\hline${ }_{3}^{4} \mathrm{P}$ & 2091.30 & 322.65 & 298.60 & 71.54 & 61.70 & 45.34 & 18.69 & 15.05 & 10.28 \\
\hline${ }_{1} \mathrm{D}$ & 2091.33 & 322.68 & 298.62 & 71.56 & 61.73 & 45.36 & 18.71 & 15.07 & 10.17 \\
\hline${ }_{3} \mathrm{D}$ & 2091.31 & 322.66 & 298.61 & 71.54 & 61.71 & 45.35 & 18.70 & 15.06 & 10.23 \\
\hline${ }_{3} \mathrm{~F}$ & 2091.32 & 322.67 & 298.61 & 71.55 & 61.72 & 45.35 & 18.70 & 15.06 & 10.21 \\
\hline${ }_{3} \mathrm{~F}$ & 2091.29 & 322.64 & 298.59 & 71.53 & 61.69 & 45.33 & 18.69 & 15.05 & 10.33 \\
\hline${ }_{3} \mathrm{G}$ & 2091.30 & 322.65 & 298.60 & 71.54 & 61.70 & 45.34 & 18.70 & 15.05 & 10.28 \\
\hline${ }_{3} \mathrm{H}$ & 2091.31 & 322.66 & 298.60 & 71.54 & 61.71 & 45.34 & 18.70 & 15.06 & 10.26 \\
\hline \hline HFS & 2156.88 & 336.92 & 309.29 & 73.29 & 63.48 & 46.52 & 18.93 & 15.71 & 10.39 \\
\hline \hline CHF & 2165.78 & 341.10 & 307.07 & 75.36 & 63.88 & 46.07 & 19.49 & 15.70 & 10.16 \\
\hline
\end{tabular}

Table A 2: Energies of Sn IX inner shells by various methods.

\begin{tabular}{|c||c|c|c|c|c|c|c|c|c|}
\hline & $1 \mathrm{~s}^{2}$ & $2 \mathrm{~s}^{2}$ & $2 \mathrm{p}^{6}$ & $3 \mathrm{~s}^{2}$ & $3 \mathrm{p}^{6}$ & $3 \mathrm{~d}^{10}$ & $4 \mathrm{~s}^{2}$ & $4 \mathrm{p}^{6}$ & $4 \mathrm{~d}^{6}$ \\
\hline \hline${ }_{0} \mathrm{~S}$ & 2093.31 & 324.67 & 300.62 & 73.48 & 63.65 & 47.29 & 20.39 & 16.69 & 11.65 \\
\hline${ }_{4} \mathrm{~S}$ & 2093.28 & 324.64 & 300.59 & 73.45 & 63.62 & 47.26 & 20.37 & 16.68 & 11.81 \\
\hline${ }_{2}{ }_{2} \mathrm{P}$ & 2093.27 & 324.63 & 300.58 & 73.45 & 63.62 & 47.26 & 20.37 & 16.68 & 11.85 \\
\hline${ }_{4}{ }_{4} \mathrm{P}$ & 2093.26 & 324.63 & 300.57 & 73.44 & 63.61 & 47.25 & 20.37 & 16.67 & 11.88 \\
\hline${ }_{2} \mathrm{D}$ & 2093.29 & 324.65 & 300.60 & 73.47 & 63.64 & 47.27 & 20.38 & 16.69 & 11.73 \\
\hline${ }_{4} \mathrm{D}$ & 2093.27 & 324.64 & 300.58 & 73.45 & 63.62 & 47.26 & 20.37 & 16.68 & 11.83 \\
\hline${ }_{4}{ }_{4} \mathrm{D}$ & 2093.26 & 324.62 & 300.57 & 73.44 & 63.61 & 47.25 & 20.37 & 16.67 & 11.89 \\
\hline${ }_{4} \mathrm{D}$ & 2093.24 & 324.60 & 300.55 & 73.42 & 63.59 & 47.23 & 20.36 & 16.66 & 12.02 \\
\hline${ }_{4} \mathrm{~F}$ & 2093.27 & 324.63 & 300.58 & 73.45 & 63.62 & 47.26 & 20.37 & 16.68 & 11.84 \\
\hline${ }_{2} \mathrm{~F}$ & 2093.27 & 324.64 & 300.58 & 73.45 & 63.62 & 47.26 & 20.37 & 16.68 & 11.83 \\
\hline${ }_{4} \mathrm{~F}$ & 2093.26 & 324.62 & 300.57 & 73.44 & 63.61 & 47.25 & 20.37 & 16.67 & 11.90 \\
\hline${ }_{2} \mathrm{G}$ & 2093.28 & 324.64 & 300.59 & 73.46 & 63.62 & 47.26 & 20.38 & 16.68 & 11.81 \\
\hline $1{ }_{4} \mathrm{G}$ & 2093.27 & 324.63 & 300.58 & 73.45 & 63.62 & 47.26 & 20.37 & 16.68 & 11.85 \\
\hline${ }_{4} \mathrm{G}$ & 2093.26 & 324.62 & 300.57 & 73.44 & 63.61 & 47.25 & 20.37 & 16.67 & 11.92 \\
\hline${ }_{4} \mathrm{H}$ & 2093.26 & 324.62 & 300.56 & 73.44 & 63.60 & 47.24 & 20.36 & 16.67 & 11.94 \\
\hline${ }_{4} \mathrm{I}$ & 2093.26 & 324.62 & 300.57 & 73.44 & 63.61 & 47.25 & 20.37 & 16.67 & 11.90 \\
\hline \hline HFS & 2158.91 & 338.97 & 311.34 & 75.26 & 65.45 & 48.50 & 20.69 & 17.44 & 12.04 \\
\hline \hline CHF & 2167.73 & 343.06 & 309.04 & 77.26 & 65.78 & 47.97 & 21.17 & 17.33 & 11.78 \\
\hline
\end{tabular}


Table A 3: Energies of $\mathrm{Sn} X$ inner shells by various methods.

\begin{tabular}{|c||c|c|c|c|c|c|c|c|c|}
\hline & $1 \mathrm{~s}^{2}$ & $2 \mathrm{~s}^{2}$ & $2 \mathrm{p}^{6}$ & $3 \mathrm{~s}^{2}$ & $3 \mathrm{p}^{6}$ & $3 \mathrm{~d}^{10}$ & $4 \mathrm{~s}^{2}$ & $4 \mathrm{p}^{6}$ & $4 \mathrm{~d}^{5}$ \\
\hline \hline${ }_{5} \mathrm{~S}$ & 2095.33 & 326.70 & 302.65 & 75.44 & 65.61 & 49.25 & 22.10 & 18.35 & 13.52 \\
\hline${ }_{5} \mathrm{~S}$ & 2095.28 & 326.66 & 302.61 & 75.40 & 65.57 & 49.21 & 22.08 & 18.33 & 13.84 \\
\hline${ }_{3} \mathrm{P}$ & 2095.35 & 326.72 & 302.67 & 75.46 & 65.63 & 49.27 & 22.11 & 18.36 & 13.36 \\
\hline${ }_{3} \mathrm{P}$ & 2095.31 & 326.68 & 302.63 & 75.42 & 65.59 & 49.23 & 22.09 & 18.34 & 13.66 \\
\hline${ }_{1} \mathrm{D}$ & 2095.35 & 326.72 & 302.67 & 75.46 & 65.63 & 49.27 & 22.11 & 18.36 & 13.38 \\
\hline${ }_{3} \mathrm{D}$ & 2095.33 & 326.71 & 302.66 & 75.45 & 65.61 & 49.25 & 22.10 & 18.35 & 13.48 \\
\hline${ }_{5} \mathrm{D}$ & 2095.33 & 326.70 & 302.65 & 75.44 & 65.61 & 49.25 & 22.10 & 18.35 & 13.53 \\
\hline${ }_{5} \mathrm{D}$ & 2095.31 & 326.68 & 302.63 & 75.43 & 65.59 & 49.23 & 22.09 & 18.34 & 13.65 \\
\hline${ }_{3} \mathrm{~F}$ & 2095.32 & 326.69 & 302.64 & 75.43 & 65.60 & 49.24 & 22.09 & 18.35 & 13.58 \\
\hline${ }_{5} \mathrm{~F}$ & 2095.33 & 326.70 & 302.65 & 75.44 & 65.61 & 49.25 & 22.10 & 18.35 & 13.55 \\
\hline${ }_{3} \mathrm{~F}$ & 2095.32 & 326.69 & 302.64 & 75.43 & 65.60 & 49.24 & 22.09 & 18.35 & 13.59 \\
\hline${ }_{3} \mathrm{G}$ & 2095.34 & 326.71 & 302.66 & 75.45 & 65.62 & 49.26 & 22.10 & 18.35 & 13.45 \\
\hline${ }_{5}^{2} \mathrm{G}$ & 2095.32 & 326.69 & 302.64 & 75.44 & 65.60 & 49.24 & 22.10 & 18.35 & 13.57 \\
\hline $4{ }_{5} \mathrm{G}$ & 2095.31 & 326.68 & 302.63 & 75.42 & 65.59 & 49.23 & 22.09 & 18.34 & 13.69 \\
\hline${ }_{3}^{2} \mathrm{H}$ & 2095.32 & 326.69 & 302.64 & 75.44 & 65.60 & 49.24 & 22.10 & 18.35 & 13.57 \\
\hline${ }_{5} \mathrm{I}$ & 2095.32 & 326.69 & 302.64 & 75.43 & 65.60 & 49.24 & 22.09 & 18.34 & 13.62 \\
\hline \hline HFS & 2161.04 & 341.11 & 313.49 & 77.32 & 67.51 & 50.57 & 22.50 & 19.22 & 13.75 \\
\hline \hline CHF & 2169.78 & 345.13 & 311.10 & 79.25 & 67.77 & 49.96 & 22.91 & 19.01 & 13.46 \\
\hline
\end{tabular}

Table A 4: Energies of Sn XI inner shells by various methods.

\begin{tabular}{|c||c|c|c|c|c|c|c|c|c|}
\hline & $1 \mathrm{~s}^{2}$ & $2 \mathrm{~s}^{2}$ & $2 \mathrm{p}^{6}$ & $3 \mathrm{~s}^{2}$ & $3 \mathrm{p}^{6}$ & $3 \mathrm{~d}^{10}$ & $4 \mathrm{~s}^{2}$ & $4 \mathrm{p}^{6}$ & $4 \mathrm{~d}^{4}$ \\
\hline \hline${ }_{0} \mathrm{~S}$ & 2097.52 & 328.90 & 304.85 & 77.54 & 67.71 & 51.36 & 23.90 & 20.09 & 14.93 \\
\hline${ }_{4} \mathrm{~S}$ & 2097.49 & 328.87 & 304.82 & 77.52 & 67.69 & 51.33 & 23.88 & 20.08 & 15.19 \\
\hline${ }_{2} \mathrm{P}$ & 2097.49 & 328.87 & 304.82 & 77.51 & 67.69 & 51.33 & 23.88 & 20.07 & 15.25 \\
\hline${ }_{4} \mathrm{P}$ & 2097.48 & 328.86 & 304.81 & 77.51 & 67.68 & 51.32 & 23.88 & 20.07 & 15.30 \\
\hline${ }_{2} \mathrm{D}$ & 2097.51 & 328.89 & 304.84 & 77.53 & 67.70 & 51.34 & 23.89 & 20.08 & 15.06 \\
\hline${ }_{4} \mathrm{D}$ & 2097.49 & 328.87 & 304.82 & 77.52 & 67.69 & 51.33 & 23.88 & 20.08 & 15.21 \\
\hline${ }_{4}^{4} \mathrm{D}$ & 2097.48 & 328.86 & 304.81 & 77.51 & 67.68 & 51.32 & 23.88 & 20.07 & 15.32 \\
\hline${ }_{4}^{4} \mathrm{D}$ & 2097.46 & 328.84 & 304.79 & 77.49 & 67.66 & 51.30 & 23.86 & 20.06 & 15.52 \\
\hline${ }_{4} \mathrm{~F}$ & 2097.49 & 328.87 & 304.82 & 77.52 & 67.69 & 51.33 & 23.88 & 20.08 & 15.23 \\
\hline${ }_{2} \mathrm{~F}$ & 2097.49 & 328.87 & 304.82 & 77.52 & 67.69 & 51.33 & 23.88 & 20.08 & 15.22 \\
\hline${ }_{4} \mathrm{~F}$ & 2097.48 & 328.86 & 304.81 & 77.51 & 67.68 & 51.32 & 23.87 & 20.07 & 15.34 \\
\hline${ }_{2} \mathrm{G}$ & 2097.49 & 328.87 & 304.82 & 77.52 & 67.69 & 51.33 & 23.88 & 20.08 & 15.19 \\
\hline${ }_{4} \mathrm{G}$ & 2097.49 & 328.87 & 304.82 & 77.51 & 67.68 & 51.33 & 23.88 & 20.07 & 15.26 \\
\hline${ }_{4}^{3} \mathrm{G}$ & 2097.47 & 328.86 & 304.81 & 77.50 & 67.68 & 51.32 & 23.87 & 20.07 & 15.36 \\
\hline${ }_{4}^{3} \mathrm{H}$ & 2097.47 & 328.85 & 304.80 & 77.50 & 67.67 & 51.32 & 23.87 & 20.07 & 15.39 \\
\hline${ }_{4}^{1} \mathrm{I}$ & 2097.48 & 328.86 & 304.81 & 77.51 & 67.68 & 51.32 & 23.88 & 20.07 & 15.33 \\
\hline \hline HFS & 2163.27 & 343.36 & 315.74 & 79.46 & 69.65 & 52.73 & 24.37 & 21.06 & 15.52 \\
\hline \hline CHF & 2171.93 & 347.29 & 313.27 & 81.32 & 69.85 & 52.04 & 24.71 & 20.74 & 15.20 \\
\hline
\end{tabular}


Table A 5: Energies of Sn XII inner shells by various methods.

\begin{tabular}{|c||c|c|c|c|c|c|c|c|c|}
\hline & $1 \mathrm{~s}^{2}$ & $2 \mathrm{~s}^{2}$ & $2 \mathrm{p}^{6}$ & $3 \mathrm{~s}^{2}$ & $3 \mathrm{p}^{6}$ & $3 \mathrm{~d}^{10}$ & $4 \mathrm{~s}^{2}$ & $4 \mathrm{p}^{6}$ & $4 \mathrm{~d}^{3}$ \\
\hline \hline${ }_{3}{ }_{3} \mathrm{P}$ & 2099.73 & 331.12 & 307.07 & 79.66 & 69.84 & 53.48 & 25.71 & 21.85 & 17.11 \\
\hline${ }_{3} \mathrm{P}$ & 2099.73 & 331.12 & 307.07 & 79.66 & 69.83 & 53.48 & 25.70 & 21.85 & 17.17 \\
\hline${ }_{1} \mathrm{D}$ & 2099.75 & 331.14 & 307.09 & 79.68 & 69.85 & 53.50 & 25.72 & 21.86 & 16.86 \\
\hline${ }_{3} \mathrm{D}$ & 2099.74 & 331.13 & 307.08 & 79.67 & 69.84 & 53.49 & 25.71 & 21.85 & 17.04 \\
\hline${ }_{3} \mathrm{~F}$ & 2099.74 & 331.13 & 307.08 & 79.67 & 69.85 & 53.49 & 25.71 & 21.85 & 16.98 \\
\hline${ }_{3} \mathrm{~F}$ & 2099.72 & 331.11 & 307.06 & 79.65 & 69.83 & 53.47 & 25.70 & 21.84 & 17.30 \\
\hline${ }_{3} \mathrm{G}$ & 2099.73 & 331.12 & 307.07 & 79.66 & 69.83 & 53.48 & 25.71 & 21.85 & 17.16 \\
\hline${ }_{3} \mathrm{H}$ & 2099.73 & 331.12 & 307.07 & 79.66 & 69.84 & 53.48 & 25.71 & 21.85 & 17.11 \\
\hline \hline HFS & 2165.59 & 345.70 & 318.08 & 81.68 & 71.88 & 54.97 & 26.29 & 22.95 & 17.34 \\
\hline \hline CHF & 2174.18 & 349.55 & 315.53 & 83.48 & 72.01 & 54.20 & 26.56 & 22.53 & 16.99 \\
\hline
\end{tabular}

Table A 6: Energies of Sn XIII inner shells by various methods.

\begin{tabular}{|c||c|c|c|c|c|c|c|c|c|}
\hline & $1 \mathrm{~s}^{2}$ & $2 \mathrm{~s}^{2}$ & $2 \mathrm{p}^{6}$ & $3 \mathrm{~s}^{2}$ & $3 \mathrm{p}^{6}$ & $3 \mathrm{~d}^{10}$ & $4 \mathrm{~s}^{2}$ & $4 \mathrm{p}^{6}$ & $4 \mathrm{~d}^{2}$ \\
\hline \hline${ }_{0} \mathrm{~S}$ & 2102.11 & 333.50 & 309.46 & 81.92 & 72.10 & 55.75 & 27.60 & 23.68 & 18.42 \\
\hline${ }_{2} \mathrm{P}$ & 2102.09 & 333.48 & 309.43 & 81.90 & 72.08 & 55.73 & 27.59 & 23.67 & 18.91 \\
\hline${ }_{2} \mathrm{D}$ & 2102.08 & 333.48 & 309.43 & 81.90 & 72.08 & 55.73 & 27.59 & 23.67 & 18.93 \\
\hline${ }_{2}^{3} \mathrm{~F}$ & 2102.08 & 333.47 & 309.43 & 81.89 & 72.07 & 55.72 & 27.59 & 23.67 & 19.11 \\
\hline${ }_{2} \mathrm{G}$ & 2102.09 & 333.48 & 309.44 & 81.90 & 72.08 & 55.73 & 27.59 & 23.68 & 18.84 \\
\hline \hline HFS & 2168.01 & 348.13 & 320.52 & 83.99 & 74.19 & 57.30 & 28.26 & 24.89 & 19.21 \\
\hline \hline CHF & 2176.53 & 351.91 & 317.88 & 85.72 & 74.24 & 56.44 & 28.46 & 24.37 & 18.84 \\
\hline
\end{tabular}




\section{APPENDIX B}

Table B 1: Calculation of Excited Sn VIII $4 d^{6} 5 p^{1}$ Energy Levels for $\mathrm{J}=0.5$ to 7.5 .

\begin{tabular}{|c|c|c|c|c|c|}
\hline & \multirow[b]{2}{*}{$\operatorname{Exp}, \mathbf{c m}^{-1}$} & \multicolumn{2}{|c|}{ HEIGHTS-ATOM (th) } & \multicolumn{2}{|c|}{ HEIGHTS-ATOM (fit) } \\
\hline & & $\begin{array}{c}\text { Energy, } \\
\text { cm }^{-1}\end{array}$ & Acc & $\begin{array}{c}\text { Energy, } \\
\text { cm }^{-1}\end{array}$ & Acc \\
\hline \multirow{21}{*}{$\begin{array}{l}\text { nم } \\
\text { II } \\
\text { In }\end{array}$} & & 583884 & & 573525 & \\
\hline & & 562919 & & 519206 & \\
\hline & & 541185 & & 512028 & \\
\hline & \multirow[t]{2}{*}{500368} & 534418 & $6.80 \%$ & 504989 & $0.92 \%$ \\
\hline & & 523367 & & 498885 & \\
\hline & 489629 & 519742 & $6.15 \%$ & 498284 & $1.77 \%$ \\
\hline & \multirow[t]{2}{*}{488019} & 517840 & $6.11 \%$ & 494478 & $1.32 \%$ \\
\hline & & 512587 & & 492732 & \\
\hline & 481842 & 509309 & $5.70 \%$ & 480701 & $0.24 \%$ \\
\hline & 477108 & 504634 & $5.77 \%$ & 473882 & $0.68 \%$ \\
\hline & 472126 & 500117 & $5.93 \%$ & 467242 & $1.03 \%$ \\
\hline & 471218 & 498509 & $5.79 \%$ & 464876 & $1.35 \%$ \\
\hline & 465949 & 495311 & $6.30 \%$ & 464272 & $0.36 \%$ \\
\hline & 461577 & 494611 & $7.16 \%$ & 463868 & $0.50 \%$ \\
\hline & 460713 & 493444 & $7.10 \%$ & 462081 & $0.30 \%$ \\
\hline & \multirow[t]{2}{*}{454426} & 490821 & $8.01 \%$ & 459414 & $1.10 \%$ \\
\hline & & 478692 & & 447917 & \\
\hline & 447257 & 467664 & $4.56 \%$ & 438583 & $1.94 \%$ \\
\hline & \multirow[t]{3}{*}{440723} & 454082 & $3.03 \%$ & 428189 & $2.84 \%$ \\
\hline & & 442601 & & 415719 & \\
\hline & & 333018 & & 397768 & \\
\hline \multirow{29}{*}{ مִ } & & 631480 & & 577523 & \\
\hline & 534686 & 576684 & $7.85 \%$ & 525889 & $1.65 \%$ \\
\hline & \multirow[t]{3}{*}{519436} & 546492 & $5.21 \%$ & 512905 & $1.26 \%$ \\
\hline & & 539107 & & 509584 & \\
\hline & & 535729 & & 507719 & \\
\hline & 504724 & 525654 & $4.15 \%$ & 504462 & $0.05 \%$ \\
\hline & 501631 & 519677 & & 502082 & \\
\hline & 499959 & 516906 & & 127 & \\
\hline & 496 & 132 & $3.05 \%$ & 5191 & $0.17 \%$ \\
\hline & 495030 & 509260 & $2.87 \%$ & 494291 & $0.15 \%$ \\
\hline & 491031 & 507777 & $3.41 \%$ & 490895 & $0.03 \%$ \\
\hline & 487686 & 506419 & $3.84 \%$ & 490597 & $0.60 \%$ \\
\hline & 486754 & 506006 & $3.96 \%$ & 486514 & $0.05 \%$ \\
\hline & 480979 & 502776 & $4.53 \%$ & 483837 & $0.59 \%$ \\
\hline & 477124 & 499717 & $4.74 \%$ & 480523 & $0.71 \%$ \\
\hline & 476998 & 498653 & $4.54 \%$ & 476597 & $0.08 \%$ \\
\hline & 475226 & 494391 & $4.03 \%$ & 468894 & $1.33 \%$ \\
\hline & 473695 & 493731 & $4.23 \%$ & 468424 & $1.11 \%$ \\
\hline & 472802 & 492453 & $4.16 \%$ & 466912 & $1.25 \%$ \\
\hline & 471728 & 488591 & $3.57 \%$ & 463961 & $1.65 \%$ \\
\hline & 468196 & 487621 & $4.15 \%$ & 463037 & $1.10 \%$ \\
\hline & 467855 & 486471 & $3.98 \%$ & 462116 & $1.23 \%$ \\
\hline & 466132 & 485388 & $4.13 \%$ & 461733 & $0.94 \%$ \\
\hline & 462285 & 484004 & $4.70 \%$ & 459771 & $0.54 \%$ \\
\hline & 460754 & 481977 & $4.61 \%$ & 459183 & $0.34 \%$ \\
\hline & 458086 & 480948 & $4.99 \%$ & 457751 & $0.07 \%$ \\
\hline & 453056 & 477237 & $5.34 \%$ & 456223 & $0.70 \%$ \\
\hline & 450221 & 475461 & $5.61 \%$ & 452745 & $0.56 \%$ \\
\hline & 446745 & 467179 & $4.57 \%$ & 446135 & $0.14 \%$ \\
\hline
\end{tabular}

\begin{tabular}{|c|c|c|c|c|c|}
\hline & \multirow{2}{*}{ Exp, $\mathbf{c m}^{-1}$} & \multicolumn{2}{|c|}{ HEIGHTS-ATOM (th) } & \multicolumn{2}{|c|}{ HEIGHTS-ATOM (fit) } \\
\hline & & $\begin{array}{c}\text { Energy, } \\
\mathrm{cm}^{-1}\end{array}$ & Acc & $\begin{array}{c}\text { Energy, } \\
\mathrm{cm}^{-1}\end{array}$ & Acc \\
\hline \multirow{6}{*}{$\begin{array}{l}\stackrel{10}{-} \\
\text { II }\end{array}$} & 445440 & 461389 & $3.58 \%$ & 438412 & $1.58 \%$ \\
\hline & & 456199 & & 432339 & \\
\hline & 440430 & 453472 & $2.96 \%$ & 430069 & $2.35 \%$ \\
\hline & & 451651 & & 424911 & \\
\hline & & 439160 & & 416616 & \\
\hline & & 428142 & & 407587 & \\
\hline \multirow{39}{*}{$\begin{array}{l}\text { م } \\
\text { N } \\
\text { II } \\
\longrightarrow\end{array}$} & 533165 & 557935 & $4.65 \%$ & 526538 & $1.24 \%$ \\
\hline & 523450 & 547626 & $4.62 \%$ & 514874 & $1.64 \%$ \\
\hline & 512335 & 536816 & $4.78 \%$ & 512414 & $0.02 \%$ \\
\hline & & 527495 & & 508299 & \\
\hline & & 523083 & & 504102 & \\
\hline & 505728 & 519536 & $2.73 \%$ & 503210 & $0.50 \%$ \\
\hline & 504726 & 518953 & $2.82 \%$ & 498045 & $1.32 \%$ \\
\hline & 500691 & 516106 & $3.08 \%$ & 496104 & $0.92 \%$ \\
\hline & 498841 & 515045 & $3.25 \%$ & 494151 & $0.94 \%$ \\
\hline & 492383 & 513207 & $4.23 \%$ & 493709 & $0.27 \%$ \\
\hline & 490580 & 510393 & $4.04 \%$ & 493641 & $0.62 \%$ \\
\hline & 489111 & 510090 & $4.29 \%$ & 491059 & $0.40 \%$ \\
\hline & 486321 & 507815 & $4.42 \%$ & 489120 & $0.58 \%$ \\
\hline & 485655 & 506014 & $4.19 \%$ & 483547 & $0.43 \%$ \\
\hline & 480770 & 505838 & $5.21 \%$ & 482445 & $0.35 \%$ \\
\hline & 478920 & 502632 & $4.95 \%$ & 480369 & $0.30 \%$ \\
\hline & 476748 & 499066 & $4.68 \%$ & 476790 & $0.01 \%$ \\
\hline & 475671 & 497944 & $4.68 \%$ & 475127 & $0.11 \%$ \\
\hline & 474586 & 497657 & $4.86 \%$ & 471899 & $0.57 \%$ \\
\hline & 473497 & 494637 & $4.46 \%$ & 470811 & $0.57 \%$ \\
\hline & 469860 & 492985 & $4.92 \%$ & 468674 & $0.25 \%$ \\
\hline & 469163 & 492757 & $5.03 \%$ & 467824 & $0.29 \%$ \\
\hline & & 491815 & & 464874 & \\
\hline & 464919 & 488818 & $5.14 \%$ & 464416 & $0.11 \%$ \\
\hline & 464306 & 488424 & $5.19 \%$ & 464265 & $0.01 \%$ \\
\hline & 463037 & 487686 & $5.32 \%$ & 463018 & $0.00 \%$ \\
\hline & 461128 & 487561 & $5.73 \%$ & 461681 & $0.12 \%$ \\
\hline & 458480 & 483930 & $5.55 \%$ & 460279 & $0.39 \%$ \\
\hline & 456344 & 482526 & $5.74 \%$ & 460051 & $0.81 \%$ \\
\hline & 455525 & 481231 & $5.64 \%$ & 459430 & $0.86 \%$ \\
\hline & 448522 & 478266 & $6.63 \%$ & 458353 & $2.19 \%$ \\
\hline & 446993 & 477250 & $6.77 \%$ & 455867 & $1.99 \%$ \\
\hline & 446190 & 472860 & $5.98 \%$ & 450700 & $1.01 \%$ \\
\hline & 443018 & 460608 & $3.97 \%$ & 436808 & $1.40 \%$ \\
\hline & 442762 & 454023 & $2.54 \%$ & 430444 & $2.78 \%$ \\
\hline & 438874 & 451703 & $2.92 \%$ & 429691 & $2.09 \%$ \\
\hline & 437418 & 445556 & $1.86 \%$ & 423489 & $3.18 \%$ \\
\hline & 424491 & 437722 & $3.12 \%$ & 417074 & $1.75 \%$ \\
\hline & & 427930 & & 407807 & \\
\hline \multirow{5}{*}{$\begin{array}{l}\text { م) } \\
\text { ले } \\
\text { II }\end{array}$} & & 557597 & & 526611 & \\
\hline & 518072 & 549816 & $6.13 \%$ & 524211 & $1.19 \%$ \\
\hline & & 526374 & & 515604 & \\
\hline & 508910 & 522933 & $2.76 \%$ & 507783 & $0.22 \%$ \\
\hline & 504960 & 520877 & $3.15 \%$ & 500794 & $0.83 \%$ \\
\hline
\end{tabular}




\begin{tabular}{|c|c|c|c|c|}
\hline \multirow{2}{*}{ Exp, cm $^{-1}$} & \multicolumn{2}{|c|}{ HEIGHTS-ATOM (th) } & \multicolumn{2}{|c|}{ HEIGHTS-ATOM (fit) } \\
\cline { 2 - 5 } & $\begin{array}{c}\text { Energy, } \\
\text { cm }^{-1}\end{array}$ & Acc & $\begin{array}{c}\text { Energy, } \\
\text { cm }^{-1}\end{array}$ & Acc \\
\hline 503073 & 515913 & $2.55 \%$ & 497733 & $1.06 \%$ \\
\hline 500095 & 514990 & $2.98 \%$ & 496876 & $0.64 \%$ \\
\hline 493417 & 512400 & $3.85 \%$ & 493332 & $0.02 \%$ \\
\hline 490476 & 511958 & $4.38 \%$ & 491506 & $0.21 \%$ \\
\hline 489608 & 508802 & $3.92 \%$ & 491394 & $0.36 \%$ \\
\hline 483311 & 508519 & $5.22 \%$ & 488191 & $1.01 \%$ \\
\hline 482684 & 508047 & $5.25 \%$ & 487167 & $0.93 \%$ \\
\hline 479577 & 506247 & $5.56 \%$ & 482413 & $0.59 \%$ \\
\hline 478651 & 498960 & $4.24 \%$ & 480729 & $0.43 \%$ \\
\hline 475560 & 498207 & $4.76 \%$ & 476618 & $0.22 \%$ \\
\hline 474209 & 496793 & $4.76 \%$ & 475006 & $0.17 \%$ \\
\hline 473750 & 493543 & $4.18 \%$ & 474712 & $0.20 \%$ \\
\hline 471667 & 491814 & $4.27 \%$ & 473714 & $0.43 \%$ \\
\hline 468623 & 489998 & $4.56 \%$ & 469676 & $0.22 \%$ \\
\hline 467779 & 489575 & $4.66 \%$ & 467527 & $0.05 \%$ \\
\hline & 489453 & & 466294 & \\
\hline II \\
\hline II
\end{tabular}

\begin{tabular}{|c|c|c|c|c|c|}
\hline & \multirow{2}{*}{$\operatorname{Exp}, \mathrm{cm}^{-1}$} & \multicolumn{2}{|c|}{ HEIGHTS-ATOM (th) } & \multicolumn{2}{|c|}{ HEIGHTS-ATOM (fit) } \\
\hline & & $\begin{array}{c}\text { Energy, } \\
\text { cm }^{-1}\end{array}$ & Acc & $\begin{array}{c}\text { Energy, } \\
\mathrm{cm}^{-1}\end{array}$ & Acc \\
\hline \multirow{17}{*}{$\begin{array}{l}n \\
\dot{f} \\
\text { II } \\
\longrightarrow\end{array}$} & 475592 & 494905 & $4.06 \%$ & 482304 & $1.41 \%$ \\
\hline & 475027 & 492628 & $3.71 \%$ & 475159 & $0.03 \%$ \\
\hline & 496026 & 489716 & $1.27 \%$ & 471724 & $4.90 \%$ \\
\hline & 468801 & 487677 & $4.03 \%$ & 469423 & $0.13 \%$ \\
\hline & 466888 & 484774 & $3.83 \%$ & 467845 & $0.21 \%$ \\
\hline & 465266 & 484395 & $4.11 \%$ & 466120 & $0.18 \%$ \\
\hline & 462784 & 481971 & $4.15 \%$ & 465145 & $0.51 \%$ \\
\hline & 458604 & 480468 & $4.77 \%$ & 464711 & $1.33 \%$ \\
\hline & 465015 & 477906 & $2.77 \%$ & 462827 & $0.47 \%$ \\
\hline & 455636 & 476373 & $4.55 \%$ & 462236 & $1.45 \%$ \\
\hline & 453389 & 476057 & $5.00 \%$ & 460635 & $1.60 \%$ \\
\hline & 449690 & 472836 & $5.15 \%$ & 460594 & $2.42 \%$ \\
\hline & 445212 & 468631 & $5.26 \%$ & 459643 & $3.24 \%$ \\
\hline & & 453068 & & 453318 & \\
\hline & 437428 & 448705 & $2.58 \%$ & 426152 & $2.58 \%$ \\
\hline & 429008 & 439241 & $2.39 \%$ & 416550 & $2.90 \%$ \\
\hline & & 417250 & & 404998 & \\
\hline \multirow{15}{*}{$\begin{array}{l}\text { م) } \\
\text { مी } \\
\text { II } \\
\text { L }\end{array}$} & & 528197 & & 525273 & \\
\hline & & 515269 & & 494389 & \\
\hline & & 510292 & & 492923 & \\
\hline & 480248 & 495052 & $3.08 \%$ & 492158 & $2.48 \%$ \\
\hline & 473754 & 493545 & $4.18 \%$ & 488672 & $3.15 \%$ \\
\hline & 470673 & 491572 & $4.44 \%$ & 481519 & $2.30 \%$ \\
\hline & 467078 & 489253 & $4.75 \%$ & 470939 & $0.83 \%$ \\
\hline & 465511 & 482078 & $3.56 \%$ & 466755 & $0.27 \%$ \\
\hline & 462536 & 480252 & $3.83 \%$ & 465206 & $0.58 \%$ \\
\hline & 458947 & 477950 & $4.14 \%$ & 464518 & $1.21 \%$ \\
\hline & 456734 & 472273 & $3.40 \%$ & 462895 & $1.35 \%$ \\
\hline & 454027 & 471168 & $3.78 \%$ & 461733 & $1.70 \%$ \\
\hline & 445145 & 469840 & $5.55 \%$ & 460613 & $3.47 \%$ \\
\hline & 443110 & 454030 & $2.46 \%$ & 454017 & $2.46 \%$ \\
\hline & & 442707 & & 416770 & \\
\hline \multirow{6}{*}{$\begin{array}{l}\text { ? } \\
0 \\
\text { II } \\
\text { ᄀ }\end{array}$} & 480421 & 497796 & $3.62 \%$ & 495274 & $3.09 \%$ \\
\hline & 468370 & 489374 & $4.48 \%$ & 487697 & $4.13 \%$ \\
\hline & 462580 & 480100 & $3.79 \%$ & 465563 & $0.64 \%$ \\
\hline & & 473009 & & 464119 & \\
\hline & 457182 & 470849 & $2.99 \%$ & 461623 & $0.97 \%$ \\
\hline & & 460326 & & 460824 & \\
\hline \multirow{2}{*}{$\stackrel{n}{\sim}$} & & 495689 & & 490111 & \\
\hline & & 472619 & & 463285 & \\
\hline
\end{tabular}




\section{APPENDIX C}

The tables that follow include the Sn IX - Sn XIII EUV transitions in the $13.5 \pm 2 \% \mathrm{~nm}$ bandwidth (13.23-13.77 nm). The energy of a transition is calculated as difference of total energies of the excited level $i$ and the ground level $j$. Columns 1 and 2 contain the total energies, column 3 shows the total moments of the source and target levels, column 5 contains the transition wavelength, and the last column contains non-dimensional oscillator strength.

Table C 1: Calculation of Sn IX $4 d^{6}-4 d^{5} 4 f^{1}$ EUV transitions.

\begin{tabular}{|c|c|c|c|c|c|}
\hline Lower level, Ry & Upper level, Ry & $J_{i}-J_{j}$ & Energy, Ry & $\lambda, \mathrm{nm}$ & $f_{i j}$ \\
\hline 12269.71 & 12276.62 & $1-2$ & 6.902232 & 13.20231 & 0.000104 \\
\hline 12269.27 & 12276.10 & $1-2$ & 6.830029 & 13.34188 & 0.000018 \\
\hline 12269.93 & 12276.68 & $4-4$ & 6.752693 & 13.49468 & 0.000424 \\
\hline 12269.27 & 12276.00 & $1-2$ & 6.736535 & 13.52705 & 0.000107 \\
\hline 12269.68 & 12276.39 & $3-4$ & 6.715597 & 13.56922 & 0.000879 \\
\hline 12269.93 & 12276.64 & $4-3$ & 6.710891 & 13.57874 & 0.006395 \\
\hline 12269.98 & 12276.68 & $5-4$ & 6.700022 & 13.60076 & 0.000076 \\
\hline 12269.70 & 12276.39 & $3-4$ & 6.693687 & 13.61364 & 0.000300 \\
\hline 12269.27 & 12275.96 & $1-2$ & 6.692024 & 13.61702 & 0.000057 \\
\hline 12270.01 & 12276.68 & $5-4$ & 6.676483 & 13.64872 & 0.000121 \\
\hline 12270.03 & 12276.68 & $3-4$ & 6.650419 & 13.70221 & 0.001019 \\
\hline 12269.98 & 12276.62 & $1-2$ & 6.640434 & 13.72281 & 0.000015 \\
\hline 12270.00 & 12276.64 & $2-3$ & 6.635490 & 13.73304 & 0.003664 \\
\hline 12269.68 & 12276.31 & $3-3$ & 6.629072 & 13.74633 & 0.009445 \\
\hline 12269.68 & 12276.30 & $3-4$ & 6.623991 & 13.75688 & 0.000517 \\
\hline 12269.68 & 12276.30 & $3-2$ & 6.618909 & 13.76744 & 0.155425 \\
\hline 12270.00 & 12276.62 & $2-2$ & 6.612253 & 13.78130 & 0.005178 \\
\hline
\end{tabular}


Table C 2: Calculation of Sn X $4 d^{5}-4 d^{4} 4 f^{1}$ EUV transitions.

\begin{tabular}{|c|c|c|c|c|c|}
\hline Lower level, Ry & Upper level, Ry & $J_{i}-J_{j}$ & Energy, Ry & $\lambda, \mathrm{nm}$ & $f_{i j}$ \\
\hline 12258.02 & 12264.91 & $2.5-3.5$ & 6.891484 & 13.22290 & 0.000090 \\
\hline 12258.42 & 12265.31 & $3.5-2.5$ & 6.882666 & 13.23984 & 0.000008 \\
\hline 12258.03 & 12264.91 & $4.5-3.5$ & 6.880646 & 13.24373 & 0.006416 \\
\hline 12258.03 & 12264.90 & $4.5-5.5$ & 6.875768 & 13.25313 & 0.000021 \\
\hline 12258.03 & 12264.90 & $4.5-4.5$ & 6.870598 & 13.26310 & 0.002518 \\
\hline 12258.44 & 12265.31 & $1.5-2.5$ & 6.865376 & 13.27319 & 0.000001 \\
\hline 12258.02 & 12264.88 & $2.5-2.5$ & 6.861611 & 13.28047 & 0.000428 \\
\hline 12258.02 & 12264.87 & $2.5-1.5$ & 6.853315 & 13.29655 & 0.000071 \\
\hline 12258.46 & 12265.31 & $2.5-2.5$ & 6.843287 & 13.31603 & 0.000005 \\
\hline 12258.46 & 12265.31 & $1.5-2.5$ & 6.841684 & 13.31915 & 0.000002 \\
\hline 12258.50 & 12265.31 & $3.5-2.5$ & 6.808647 & 13.38378 & 0.000005 \\
\hline 12258.02 & 12264.81 & $2.5-3.5$ & 6.797375 & 13.40597 & 0.000138 \\
\hline 12258.51 & 12265.31 & $2.5-2.5$ & 6.795462 & 13.40975 & 0.000001 \\
\hline 12258.03 & 12264.81 & $4.5-3.5$ & 6.786537 & 13.42738 & 0.003027 \\
\hline 12258.52 & 12265.31 & $1.5-2.5$ & 6.782472 & 13.43543 & 0.000008 \\
\hline 12258.52 & 12265.31 & $3.5-2.5$ & 6.781388 & 13.43758 & 0.000010 \\
\hline 12258.15 & 12264.92 & $2.5-2.5$ & 6.771324 & 13.45755 & 0.000122 \\
\hline 12258.16 & 12264.92 & $3.5-2.5$ & 6.769100 & 13.46197 & 0.000056 \\
\hline 12258.54 & 12265.31 & $1.5-2.5$ & 6.765783 & 13.46857 & 0.000002 \\
\hline 12258.16 & 12264.92 & $1.5-2.5$ & 6.759534 & 13.48102 & 0.000465 \\
\hline 12258.17 & 12264.92 & 2.5-2.5 & 6.755462 & 13.48915 & 0.000001 \\
\hline 12258.15 & 12264.91 & $2.5-3.5$ & 6.755014 & 13.49004 & 0.000152 \\
\hline 12258.16 & 12264.91 & $3.5-3.5$ & 6.752790 & 13.49448 & 0.000054 \\
\hline 12258.02 & 12264.76 & $2.5-1.5$ & 6.745445 & 13.50918 & 0.000561 \\
\hline 12258.17 & 12264.91 & $2.5-3.5$ & 6.739152 & 13.52179 & 0.000011 \\
\hline 12258.15 & 12264.88 & $2.5-2.5$ & 6.725141 & 13.54996 & 0.000044 \\
\hline 12258.16 & 12264.88 & $3.5-2.5$ & 6.722917 & 13.55445 & 0.000068 \\
\hline 12258.58 & 12265.31 & $2.5-2.5$ & 6.722120 & 13.55605 & 0.000013 \\
\hline 12258.58 & 12265.31 & $3.5-2.5$ & 6.720895 & 13.55852 & 0.000004 \\
\hline 12258.15 & 12264.87 & 2.5-1.5 & 6.716845 & 13.56670 & 0.000029 \\
\hline 12258.16 & 12264.88 & $1.5-2.5$ & 6.713351 & 13.57376 & 0.000063 \\
\hline
\end{tabular}




\begin{tabular}{|c|c|c|c|c|c|}
\hline 12258.03 & 12264.74 & $4.5-5.5$ & 6.711484 & 13.57754 & 0.000071 \\
\hline 12258.17 & 12264.88 & $2.5-2.5$ & 6.709279 & 13.58200 & 0.000012 \\
\hline 12258.16 & 12264.87 & $1.5-1.5$ & 6.705055 & 13.59055 & 0.000001 \\
\hline 12258.61 & 12265.31 & $3.5-2.5$ & 6.699278 & 13.60227 & 0.000011 \\
\hline 12258.61 & 12265.31 & $2.5-2.5$ & 6.695434 & 13.61008 & 0.000001 \\
\hline 12258.61 & 12265.31 & $3.5-2.5$ & 6.691390 & 13.61831 & 0.000003 \\
\hline 12258.63 & 12265.31 & $2.5-2.5$ & 6.679497 & 13.64256 & 0.000002 \\
\hline 12258.63 & 12265.31 & 3.5-2.5 & 6.676075 & 13.64955 & 0.000014 \\
\hline 12258.63 & 12265.31 & 2.5-2.5 & 6.673786 & 13.65423 & 0.000002 \\
\hline 12258.25 & 12264.92 & $2.5-2.5$ & 6.671947 & 13.65799 & 0.000805 \\
\hline 12258.64 & 12265.31 & $1.5-2.5$ & 6.666580 & 13.66899 & 0.000002 \\
\hline 12258.15 & 12264.81 & 2.5-3.5 & 6.660905 & 13.68064 & 0.000015 \\
\hline 12258.65 & 12265.31 & $3.5-2.5$ & 6.659999 & 13.68250 & 0.000012 \\
\hline 12258.65 & 12265.31 & 3.5-2.5 & 6.658693 & 13.68518 & 0.000002 \\
\hline 12258.16 & 12264.81 & 3.5-3.5 & 6.658681 & 13.68521 & 0.000006 \\
\hline 12258.27 & 12264.92 & $3.5-2.5$ & 6.655962 & 13.69080 & 0.000001 \\
\hline 12258.25 & 12264.91 & 2.5-3.5 & 6.655637 & 13.69146 & 0.000034 \\
\hline 12258.02 & 12264.66 & 2.5-3.5 & 6.646327 & 13.71064 & 0.000674 \\
\hline 12258.17 & 12264.81 & 2.5-3.5 & 6.645043 & 13.71329 & 0.000003 \\
\hline 12258.28 & 12264.92 & $1.5-2.5$ & 6.640648 & 13.72237 & 0.000026 \\
\hline 12258.27 & 12264.91 & 3.5-3.5 & 6.639652 & 13.72443 & 0.000739 \\
\hline 12258.03 & 12264.66 & 4.5-3.5 & 6.635489 & 13.73304 & 0.001069 \\
\hline 12258.29 & 12264.92 & $2.5-2.5$ & 6.634390 & 13.73531 & 0.000192 \\
\hline 12258.03 & 12264.66 & 4.5-4.5 & 6.631867 & 13.74054 & 0.000982 \\
\hline 12258.68 & 12265.31 & $3.5-2.5$ & 6.630319 & 13.74375 & 0.000006 \\
\hline 12258.25 & 12264.88 & 2.5-2.5 & 6.625764 & 13.75319 & 0.000755 \\
\hline 12258.30 & 12264.92 & $3.5-2.5$ & 6.625658 & 13.75341 & 0.000123 \\
\hline 12258.30 & 12264.92 & $1.5-2.5$ & 6.623227 & 13.75846 & 0.000087 \\
\hline 12258.68 & 12265.31 & $3.5-2.5$ & 6.622783 & 13.75938 & 0.000003 \\
\hline 12258.69 & 12265.31 & 2.5-2.5 & 6.619029 & 13.76719 & 0.000004 \\
\hline 12258.69 & 12265.31 & 3.5-2.5 & 6.618500 & 13.76829 & 0.000006 \\
\hline 12258.29 & 12264.91 & 2.5-3.5 & 6.618080 & 13.76916 & 0.000265 \\
\hline 12258.02 & 12264.63 & $2.5-2.5$ & 6.617922 & 13.76949 & 0.001654 \\
\hline 12258.25 & 12264.87 & $2.5-1.5$ & 6.617468 & 13.77044 & 0.000397 \\
\hline
\end{tabular}


Table C 3: Calculation of Sn X $4 d^{5}-4 d^{4} 5 p^{1}$ EUV transitions.

\begin{tabular}{|c|c|c|c|c|c|}
\hline Lower level, Ry & Upper level, Ry & $J_{i}-J_{j}$ & Energy, Ry & $\lambda, \mathrm{nm}$ & $f_{i j}$ \\
\hline 12257.49 & 12264.43 & $1.5-2.5$ & 6.940224 & 13.13004 & 0.001501 \\
\hline 12257.49 & 12264.34 & $1.5-1.5$ & 6.847644 & 13.30756 & 0.000050 \\
\hline 12257.49 & 12264.32 & $1.5-2.5$ & 6.825849 & 13.35005 & 0.000129 \\
\hline 12257.49 & 12264.09 & $1.5-1.5$ & 6.596839 & 13.81350 & 0.000374 \\
\hline
\end{tabular}

Table C 4: Calculation of Sn XI $4 d^{4}-4 d^{3} 4 f^{1}$ EUV transitions.

\begin{tabular}{|c|c|c|c|c|c|}
\hline Lower level, Ry & Upper level, Ry & $J_{i}-J_{j}$ & Energy, Ry & $\lambda, \mathrm{nm}$ & $f_{i j}$ \\
\hline 12244.80 & 12251.69 & $3-4$ & 6.894059 & 13.21796 & 0.000055 \\
\hline 12244.81 & 12251.69 & $3-4$ & 6.883747 & 13.23776 & 0.000058 \\
\hline 12244.88 & 12251.76 & $3-2$ & 6.883176 & 13.23886 & 0.000508 \\
\hline 12244.81 & 12251.69 & $5-4$ & 6.883083 & 13.23904 & 0.004176 \\
\hline 12244.88 & 12251.76 & $2-2$ & 6.882066 & 13.24100 & 0.000015 \\
\hline 12244.88 & 12251.76 & $1-2$ & 6.879515 & 13.24591 & 0.003504 \\
\hline 12244.91 & 12251.79 & $1-1$ & 6.873717 & 13.25708 & 0.000053 \\
\hline 12244.14 & 12251.02 & $1-2$ & 6.873667 & 13.25718 & 0.000369 \\
\hline 12244.51 & 12251.39 & $5-4$ & 6.873604 & 13.25730 & 0.001224 \\
\hline 12244.47 & 12251.34 & $3-2$ & 6.872004 & 13.26038 & 0.002656 \\
\hline 12244.47 & 12251.33 & $3-4$ & 6.864104 & 13.27565 & 0.000305 \\
\hline 12244.47 & 12251.33 & $3-3$ & 6.863995 & 13.27586 & 0.000018 \\
\hline 12244.83 & 12251.69 & $4-4$ & 6.863483 & 13.27685 & 0.000253 \\
\hline 12244.86 & 12251.73 & $2-3$ & 6.863419 & 13.27697 & 0.000025 \\
\hline 12244.90 & 12251.76 & $3-2$ & 6.860414 & 13.28279 & 0.000159 \\
\hline 12244.47 & 12251.32 & $3-2$ & 6.856421 & 13.29052 & 0.000599 \\
\hline 12244.90 & 12251.76 & $1-2$ & 6.855721 & 13.29188 & 0.000322 \\
\hline 12244.88 & 12251.73 & $3-3$ & 6.851211 & 13.30063 & 0.001243 \\
\hline 12244.88 & 12251.73 & $2-3$ & 6.850101 & 13.30278 & 0.000491 \\
\hline 12244.94 & 12251.79 & $2-1$ & 6.848238 & 13.30640 & 0.000004 \\
\hline 12244.91 & 12251.76 & $1-2$ & 6.847593 & 13.30766 & 0.001264 \\
\hline & & & & & \\
\hline
\end{tabular}




\begin{tabular}{|c|c|c|c|c|c|}
\hline 12244.92 & 12251.76 & $3-2$ & 6.836741 & 13.32878 & 0.000025 \\
\hline 12244.90 & 12251.73 & 3-3 & 6.828449 & 13.34497 & 0.002593 \\
\hline 12244.94 & 12251.76 & $2-2$ & 6.822114 & 13.35736 & 0.001967 \\
\hline 12244.51 & 12251.33 & $5-4$ & 6.819337 & 13.36280 & 0.017153 \\
\hline 12244.47 & 12251.29 & 3-3 & 6.818086 & 13.36525 & 0.003586 \\
\hline 12244.88 & 12251.69 & $3-4$ & 6.814937 & 13.37142 & 0.000004 \\
\hline 12244.69 & 12251.50 & $5-4$ & 6.812768 & 13.37568 & 0.012189 \\
\hline 12244.92 & 12251.73 & 3-3 & 6.804776 & 13.39139 & 0.000172 \\
\hline 12244.71 & 12251.50 & $5-4$ & 6.793209 & 13.41419 & 0.000441 \\
\hline 12244.94 & 12251.73 & $4-3$ & 6.793038 & 13.41453 & 0.000032 \\
\hline 12244.90 & 12251.69 & $3-4$ & 6.792175 & 13.41624 & 0.000459 \\
\hline 12244.94 & 12251.73 & $2-3$ & 6.790149 & 13.42024 & 0.000633 \\
\hline 12245.00 & 12251.79 & $2-1$ & 6.788984 & 13.42254 & 0.000334 \\
\hline 12244.98 & 12251.76 & $3-2$ & 6.784687 & 13.43104 & 0.000356 \\
\hline 12244.69 & 12251.47 & $5-5$ & 6.778569 & 13.44316 & 0.015460 \\
\hline 12244.92 & 12251.69 & $3-4$ & 6.768502 & 13.46316 & 0.000071 \\
\hline 12244.96 & 12251.73 & $4-3$ & 6.764406 & 13.47131 & 0.001263 \\
\hline 12245.00 & 12251.76 & $2-2$ & 6.762860 & 13.47439 & 0.000119 \\
\hline 12244.47 & 12251.23 & $3-2$ & 6.757768 & 13.48454 & 0.004950 \\
\hline 12244.94 & 12251.69 & $4-4$ & 6.756764 & 13.48655 & 0.000602 \\
\hline 12245.00 & 12251.76 & $3-2$ & 6.756106 & 13.48786 & 0.000226 \\
\hline 12244.98 & 12251.73 & $3-3$ & 6.752722 & 13.49462 & 0.000097 \\
\hline 12244.69 & 12251.44 & $5-6$ & 6.751473 & 13.49712 & 0.000906 \\
\hline 12244.95 & 12251.69 & $5-4$ & 6.745791 & 13.50849 & 0.003608 \\
\hline 12244.99 & 12251.73 & $4-3$ & 6.741759 & 13.51656 & 0.002842 \\
\hline 12245.05 & 12251.79 & $2-1$ & 6.73515 & 13.52983 & 0.000039 \\
\hline 12244.61 & 12251.34 & $1-2$ & 6.733177 & 13.53379 & 0.000329 \\
\hline 12245.00 & 12251.73 & $2-3$ & 6.730895 & 13.53838 & 0.000024 \\
\hline 12244.77 & 12251.50 & $3-4$ & 6.728196 & 13.54381 & 0.000407 \\
\hline 12244.96 & 12251.69 & $4-4$ & 6.728132 & 13.54394 & 0.000021 \\
\hline 12244.70 & 12251.43 & $2-3$ & 6.727805 & 13.54460 & 0.001737 \\
\hline 12245.00 & 12251.73 & 3-3 & 6.724141 & 13.55198 & 0.002877 \\
\hline 12244.61 & 12251.32 & $1-2$ & 6.717594 & 13.56519 & 0.000322 \\
\hline 12244.98 & 12251.69 & $3-4$ & 6.716448 & 13.56750 & 0.000117 \\
\hline 12245.05 & 12251.76 & $1-2$ & 6.710335 & 13.57986 & 0.000043 \\
\hline
\end{tabular}




\begin{tabular}{|c|c|c|c|c|c|}
\hline 12245.05 & 12251.76 & $2-2$ & 6.709026 & 13.58251 & 0.000138 \\
\hline 12245.05 & 12251.76 & $3-2$ & 6.70801 & 13.58457 & 0.000025 \\
\hline 12244.73 & 12251.44 & $7-6$ & 6.707233 & 13.58614 & 0.169773 \\
\hline 12244.99 & 12251.69 & $4-4$ & 6.705485 & 13.58968 & 0.002073 \\
\hline 12245.02 & 12251.73 & $4-3$ & 6.704517 & 13.59165 & 0.006088 \\
\hline 12244.80 & 12251.50 & $3-4$ & 6.703394 & 13.59392 & 0.000145 \\
\hline 12244.47 & 12251.17 & $3-4$ & 6.698364 & 13.60413 & 0.004650 \\
\hline 12244.69 & 12251.39 & $5-4$ & 6.697115 & 13.60667 & 0.002026 \\
\hline 12244.81 & 12251.50 & $3-4$ & 6.693082 & 13.61487 & 0.000006 \\
\hline 12244.81 & 12251.50 & $5-4$ & 6.692418 & 13.61622 & 0.001374 \\
\hline 12245.00 & 12251.69 & $3-4$ & 6.687867 & 13.62548 & 0.000134 \\
\hline 12245.07 & 12251.76 & $2-2$ & 6.685994 & 13.62930 & 0.000014 \\
\hline 12245.08 & 12251.76 & $3-2$ & 6.679984 & 13.64156 & 0.000071 \\
\hline 12244.71 & 12251.39 & $5-4$ & 6.677556 & 13.64652 & 0.025702 \\
\hline 12245.05 & 12251.73 & $2-3$ & 6.677061 & 13.64753 & 0.001226 \\
\hline 12245.05 & 12251.73 & 3-3 & 6.676045 & 13.64961 & 0.000421 \\
\hline 12244.83 & 12251.5 & $4-4$ & 6.672818 & 13.65621 & 0.000005 \\
\hline 12245.09 & 12251.76 & $1-2$ & 6.672154 & 13.65757 & 0.000005 \\
\hline 12245.02 & 12251.69 & 4-4 & 6.668243 & 13.66558 & 0.003290 \\
\hline 12244.47 & 12251.13 & $3-2$ & 6.664878 & 13.67248 & 0.001852 \\
\hline 12245.07 & 12251.73 & $2-3$ & 6.654029 & 13.69477 & 0.000078 \\
\hline 12244.77 & 12251.43 & $3-3$ & 6.653639 & 13.69558 & 0.002406 \\
\hline 12244.51 & 12251.17 & $5-4$ & 6.653597 & 13.69566 & 0.000612 \\
\hline 12245.08 & 12251.73 & $4-3$ & 6.652822 & 13.69726 & 0.000255 \\
\hline 12245.14 & 12251.79 & $2-1$ & 6.650296 & 13.70246 & 0.000002 \\
\hline 12245.08 & 12251.73 & 3-3 & 6.648019 & 13.70715 & 0.005865 \\
\hline 12244.69 & 12251.33 & $5-4$ & 6.642848 & 13.71782 & 0.046407 \\
\hline 12244.47 & 12251.11 & $3-3$ & 6.640605 & 13.72246 & 0.015694 \\
\hline 12244.70 & 12251.34 & $2-2$ & 6.640342 & 13.72300 & 0.024252 \\
\hline 12244.69 & 12251.33 & $5-5$ & 6.639916 & 13.72388 & 0.000491 \\
\hline 12245.05 & 12251.69 & $3-4$ & 6.639771 & 13.72418 & 0.002333 \\
\hline 12244.83 & 12251.47 & $4-5$ & 6.638619 & 13.72656 & 0.000071 \\
\hline 12245.12 & 12251.76 & $3-2$ & 6.636858 & 13.73020 & 0.000125 \\
\hline 12245.15 & 12251.79 & $1-1$ & 6.636624 & 13.73069 & 0.000243 \\
\hline 12245.06 & 12251.69 & $5-4$ & 6.633555 & 13.73704 & 0.003798 \\
\hline
\end{tabular}




\begin{tabular}{|l|l|l|l|l|l|}
\hline 12244.70 & 12251.33 & $2-3$ & 6.632333 & 13.73957 & 0.004531 \\
\hline 12245.13 & 12251.76 & $3-2$ & 6.630589 & 13.74319 & 0.000848 \\
\hline 12244.80 & 12251.43 & $3-3$ & 6.628837 & 13.74682 & 0.000008 \\
\hline 12245.10 & 12251.73 & $4-3$ & 6.626732 & 13.75118 & 0.013451 \\
\hline 12244.70 & 12251.32 & $2-2$ & 6.624759 & 13.75528 & 0.014526 \\
\hline 12244.88 & 12251.50 & $3-4$ & 6.624272 & 13.75629 & 0.000004 \\
\hline 12245.14 & 12251.76 & $2-2$ & 6.624172 & 13.75650 & 0.000779 \\
\hline 12244.47 & 12251.09 & $3-2$ & 6.624058 & 13.75674 & 0.117769 \\
\hline 12244.71 & 12251.33 & $5-4$ & 6.623289 & 13.75833 & 0.002178 \\
\hline 12244.61 & 12251.23 & $1-2$ & 6.618941 & 13.76737 & 0.000592 \\
\hline 12244.81 & 12251.43 & $3-3$ & 6.618525 & 13.76824 & 0.002580 \\
\hline 12245.08 & 12251.69 & $4-4$ & 6.616548 & 13.77235 & 0.001870 \\
\hline 12244.77 & 12251.39 & $3-4$ & 6.612543 & 13.78069 & 0.001295 \\
\hline
\end{tabular}

Table C 5: Calculation of Sn XI $4 d^{4}-4 d^{3} 5 p^{1}$ EUV transitions.

\begin{tabular}{|c|c|c|c|c|c|}
\hline Lower level, Ry & Upper level, Ry & $J_{i}-J_{j}$ & Energy, Ry & $\lambda, \mathrm{nm}$ & $f_{i j}$ \\
\hline 12244.87 & 12251.76 & $2-2$ & 6.889793 & 13.22615 & 0.000571 \\
\hline 12244.56 & 12251.43 & $3-3$ & 6.868948 & 13.26628 & 0.000006 \\
\hline 12244.82 & 12251.69 & $3-4$ & 6.868550 & 13.26705 & 0.000226 \\
\hline 12244.86 & 12251.73 & $3-3$ & 6.868095 & 13.26793 & 0.000245 \\
\hline 12244.87 & 12251.73 & $2-3$ & 6.857828 & 13.28780 & 0.000018 \\
\hline 12244.92 & 12251.76 & $2-2$ & 6.844043 & 13.31456 & 0.000002 \\
\hline 12244.86 & 12251.69 & $3-4$ & 6.831821 & 13.33838 & 0.000156 \\
\hline 12244.93 & 12251.76 & $2-2$ & 6.829306 & 13.34329 & 0.000011 \\
\hline 12244.56 & 12251.39 & $3-4$ & 6.827852 & 13.34613 & 0.001513 \\
\hline 12244.94 & 12251.76 & $1-2$ & 6.824630 & 13.35243 & 0.000002 \\
\hline 12244.92 & 12251.73 & $2-3$ & 6.812078 & 13.37704 & 0.000015 \\
\hline 12244.96 & 12251.76 & $1-2$ & 6.801678 & 13.39749 & 0.000001 \\
\hline 12244.93 & 12251.73 & $2-3$ & 6.797341 & 13.40604 & 0.000109 \\
\hline 12244.99 & 12251.79 & $2-1$ & 6.795518 & 13.40964 & 0.000063 \\
\hline 12244.56 & 12251.34 & $3-2$ & 6.781485 & 13.43738 & 0.000179 \\
\hline 12245.01 & 12251.79 & $1-1$ & 6.777861 & 13.44457 & 0.000008 \\
\hline 12244.73 & 12251.50 & $4-4$ & 6.773639 & 13.45295 & 0.000054 \\
\hline
\end{tabular}




\begin{tabular}{|c|c|c|c|c|c|}
\hline 12244.56 & 12251.33 & $3-4$ & 6.773585 & 13.45306 & 0.000474 \\
\hline 12244.56 & 12251.33 & 3-3 & 6.773476 & 13.45327 & 0.000496 \\
\hline 12244.99 & 12251.76 & $2-2$ & 6.769394 & 13.46138 & 0.000099 \\
\hline 12244.56 & 12251.32 & $3-2$ & 6.765902 & 13.46833 & 0.000066 \\
\hline 12243.96 & 12250.72 & $2-2$ & 6.758530 & 13.48302 & 0.019647 \\
\hline 12245.01 & 12251.76 & $3-2$ & 6.752213 & 13.49564 & 0.000040 \\
\hline 12245.01 & 12251.76 & $1-2$ & 6.751737 & 13.49659 & 0.000014 \\
\hline 12245.04 & 12251.79 & $1-1$ & 6.750366 & 13.49933 & 0.000020 \\
\hline 12245.04 & 12251.79 & $2-1$ & 6.749810 & 13.50044 & 0.000003 \\
\hline 12245.02 & 12251.76 & $3-2$ & 6.737754 & 13.52460 & 0.000078 \\
\hline 12244.99 & 12251.73 & $2-3$ & 6.737429 & 13.52525 & 0.000068 \\
\hline 12244.96 & 12251.69 & $5-4$ & 6.733948 & 13.53224 & 0.000358 \\
\hline 12244.56 & 12251.29 & 3-3 & 6.727567 & 13.54508 & 0.000527 \\
\hline 12245.00 & 12251.73 & $4-3$ & 6.723701 & 13.55287 & 0.000042 \\
\hline 12245.04 & 12251.76 & $2-2$ & 6.723686 & 13.55290 & 0.000058 \\
\hline 12245.01 & 12251.73 & 3-3 & 6.720248 & 13.55983 & 0.000074 \\
\hline 12244.98 & 12251.69 & $5-4$ & 6.709323 & 13.58191 & 0.000016 \\
\hline 12245.02 & 12251.73 & 3-3 & 6.705789 & 13.58907 & 0.000064 \\
\hline 12244.73 & 12251.43 & $4-3$ & 6.699082 & 13.60267 & 0.000584 \\
\hline 12244.65 & 12251.34 & $1-2$ & 6.693249 & 13.61453 & 0.000273 \\
\hline 12245.04 & 12251.73 & $2-3$ & 6.691721 & 13.61764 & 0.000015 \\
\hline 12245.07 & 12251.76 & $3-2$ & 6.690117 & 13.62090 & 0.000009 \\
\hline 12245.00 & 12251.69 & $4-4$ & 6.687427 & 13.62638 & 0.000306 \\
\hline 12245.01 & 12251.69 & $3-4$ & 6.683974 & 13.63342 & 0.000067 \\
\hline 12245.10 & 12251.79 & $1-1$ & 6.681993 & 13.63746 & 0.000003 \\
\hline 12244.82 & 12251.50 & $3-4$ & 6.677885 & 13.64585 & 0.002136 \\
\hline 12244.65 & 12251.32 & $1-2$ & 6.677666 & 13.64630 & 0.000816 \\
\hline 12244.65 & 12251.32 & $1-1$ & 6.673463 & 13.65489 & 0.000026 \\
\hline 12245.02 & 12251.69 & $4-4$ & 6.670146 & 13.66168 & 0.000014 \\
\hline 12245.02 & 12251.69 & $3-4$ & 6.669515 & 13.66298 & 0.000040 \\
\hline 12245.06 & 12251.73 & $4-3$ & 6.669084 & 13.66386 & 0.000111 \\
\hline 12244.56 & 12251.23 & $3-2$ & 6.667249 & 13.66762 & 0.006923 \\
\hline 12245.10 & 12251.76 & $3-2$ & 6.660088 & 13.68231 & 0.000002 \\
\hline 12245.13 & 12251.79 & $1-1$ & 6.658900 & 13.68476 & 0.000031 \\
\hline 12245.07 & 12251.73 & 3-3 & 6.658152 & 13.68629 & 0.000093 \\
\hline
\end{tabular}




\begin{tabular}{|l|l|l|l|l|l|}
\hline 12244.73 & 12251.39 & $4-4$ & 6.657986 & 13.68663 & 0.000172 \\
\hline 12245.10 & 12251.76 & $1-2$ & 6.655869 & 13.69099 & 0.000046 \\
\hline 12245.12 & 12251.76 & $3-2$ & 6.644018 & 13.71541 & 0.000252 \\
\hline 12244.86 & 12251.50 & $3-4$ & 6.641156 & 13.72132 & 0.000184 \\
\hline 12245.06 & 12251.69 & $4-4$ & 6.632810 & 13.73858 & 0.000129 \\
\hline 12245.13 & 12251.76 & $1-2$ & 6.632776 & 13.73865 & 0.000036 \\
\hline 12245.15 & 12251.79 & $2-1$ & 6.631860 & 13.74055 & 0.000004 \\
\hline 12245.13 & 12251.76 & $3-2$ & 6.630311 & 13.74376 & 0.000049 \\
\hline 12245.10 & 12251.73 & $4-3$ & 6.630109 & 13.74418 & 0.000310 \\
\hline 12245.16 & 12251.79 & $1-1$ & 6.629847 & 13.74472 & 0.000008 \\
\hline 12245.10 & 12251.73 & $3-3$ & 6.628123 & 13.74830 & 0.000001 \\
\hline 12245.13 & 12251.76 & $3-2$ & 6.625887 & 13.75294 & 0.000002 \\
\hline 12245.14 & 12251.76 & $3-2$ & 6.625016 & 13.75475 & 0.000299 \\
\hline 12245.16 & 12251.79 & $2-1$ & 6.624865 & 13.75506 & 0.000059 \\
\hline 12245.07 & 12251.69 & $3-4$ & 6.621878 & 13.76126 & 0.000045 \\
\hline 12245.12 & 12251.73 & $3-3$ & 6.612053 & 13.78171 & 0.000006 \\
\hline
\end{tabular}

Table C 6: Calculation of Sn XII $4 d^{3}-4 d^{2} 4 f^{1}$ EUV transitions.

\begin{tabular}{|c|c|c|c|c|c|}
\hline Lower level, Ry & Upper level, Ry & $J_{i}-J_{j}$ & Energy, Ry & $\lambda, \mathrm{nm}$ & $f_{i j}$ \\
\hline 12229.53 & 12236.42 & $2.5-3.5$ & 6.893386 & 13.21925 & 0.000002 \\
\hline 12229.64 & 12236.52 & $1.5-1.5$ & 6.872908 & 13.25864 & 0.000001 \\
\hline 12229.65 & 12236.52 & $2.5-1.5$ & 6.872330 & 13.25976 & 0.000144 \\
\hline 12229.52 & 12236.37 & $3.5-4.5$ & 6.857156 & 13.28910 & 0.000002 \\
\hline 12229.38 & 12236.22 & $2.5-3.5$ & 6.835726 & 13.33076 & 0.000031 \\
\hline 12229.65 & 12236.47 & $2.5-2.5$ & 6.826796 & 13.34820 & 0.000144 \\
\hline 12229.40 & 12236.22 & $3.5-3.5$ & 6.819391 & 13.36269 & 0.000012 \\
\hline 12229.61 & 12236.42 & $4.5-3.5$ & 6.816819 & 13.36773 & 0.001502 \\
\hline 12229.46 & 12236.28 & $1.5-1.5$ & 6.815616 & 13.37009 & 0.000053 \\
\hline 12229.38 & 12236.19 & $2.5-2.5$ & 6.811084 & 13.37899 & 0.000489 \\
\hline 12229.40 & 12236.21 & $3.5-4.5$ & 6.808115 & 13.38482 & 0.000006 \\
\hline 12229.40 & 12236.19 & $3.5-2.5$ & 6.794749 & 13.41115 & 0.000735 \\
\hline 12229.74 & 12236.52 & $2.5-1.5$ & 6.776738 & 13.44680 & 0.000113 \\
\hline 12229.65 & 12236.42 & $2.5-3.5$ & 6.776393 & 13.44748 & 0.000290 \\
\hline
\end{tabular}




\begin{tabular}{|c|c|c|c|c|c|}
\hline 12229.60 & 12236.37 & $5.5-4.5$ & 6.772770 & 13.45467 & 0.008908 \\
\hline 12229.61 & 12236.37 & $4.5-4.5$ & 6.767768 & 13.46462 & 0.000022 \\
\hline 12229.53 & 12236.28 & $2.5-1.5$ & 6.747018 & 13.50603 & 0.000093 \\
\hline 12229.63 & 12236.37 & $5.5-4.5$ & 6.740409 & 13.51927 & 0.058735 \\
\hline 12229.46 & 12236.19 & $1.5-2.5$ & 6.733121 & 13.53390 & 0.000259 \\
\hline 12229.74 & 12236.47 & $2.5-2.5$ & 6.731204 & 13.53776 & 0.000088 \\
\hline 12229.38 & 12236.10 & $2.5-1.5$ & 6.715639 & 13.56914 & 0.000748 \\
\hline 12229.76 & 12236.47 & $3.5-2.5$ & 6.712212 & 13.57606 & 0.000345 \\
\hline 12229.81 & 12236.52 & $2.5-1.5$ & 6.702884 & 13.59496 & 0.000012 \\
\hline 12229.52 & 12236.22 & $3.5-3.5$ & 6.701986 & 13.59678 & 0.000086 \\
\hline 12229.52 & 12236.21 & $3.5-4.5$ & 6.690710 & 13.61969 & 0.000009 \\
\hline 12229.73 & 12236.42 & $4.5-3.5$ & 6.689327 & 13.62251 & 0.000743 \\
\hline 12229.53 & 12236.22 & $2.5-3.5$ & 6.689165 & 13.62284 & 0.000025 \\
\hline 12229.74 & 12236.42 & $2.5-3.5$ & 6.680801 & 13.63989 & 0.000013 \\
\hline 12229.84 & 12236.52 & $2.5-1.5$ & 6.677780 & 13.64606 & 0.000169 \\
\hline 12229.52 & 12236.19 & $3.5-2.5$ & 6.677344 & 13.64696 & 0.000345 \\
\hline 12229.53 & 12236.19 & $2.5-2.5$ & 6.664523 & 13.67321 & 0.000085 \\
\hline 12229.76 & 12236.42 & $3.5-3.5$ & 6.661809 & 13.67878 & 0.000460 \\
\hline 12229.86 & 12236.52 & $1.5-1.5$ & 6.660355 & 13.68177 & 0.000045 \\
\hline 12229.81 & 12236.47 & $2.5-2.5$ & 6.657350 & 13.68794 & 0.003233 \\
\hline 12229.87 & 12236.52 & $1.5-1.5$ & 6.646379 & 13.71054 & 0.000005 \\
\hline 12229.83 & 12236.47 & $3.5-2.5$ & 6.637821 & 13.72821 & 0.000060 \\
\hline 12229.46 & 12236.10 & $1.5-1.5$ & 6.637676 & 13.72851 & 0.002329 \\
\hline 12229.84 & 12236.47 & $2.5-2.5$ & 6.632246 & 13.73975 & 0.000480 \\
\hline 12229.64 & 12236.28 & $1.5-1.5$ & 6.630603 & 13.74316 & 0.000001 \\
\hline 12229.65 & 12236.28 & $2.5-1.5$ & 6.630025 & 13.74436 & 0.000212 \\
\hline 12229.89 & 12236.52 & 2.5-1.5 & 6.629385 & 13.74568 & 0.000011 \\
\hline 12229.38 & 12236.00 & $2.5-2.5$ & 6.621366 & 13.76233 & 0.026480 \\
\hline 12229.86 & 12236.47 & $1.5-2.5$ & 6.614821 & 13.77595 & 0.000238 \\
\hline
\end{tabular}


Table C 7: Calculation of Sn XII $4 d^{3}-4 d^{2} 5 p^{1}$ EUV transitions.

\begin{tabular}{|c|c|c|c|c|c|}
\hline Lower level, Ry & Upper level, Ry & $J_{i}-J_{j}$ & Energy, Ry & $\lambda, \mathrm{nm}$ & $f_{i j}$ \\
\hline 12229.21 & 12236.09 & $5.5-5.5$ & 6.889494 & 13.22672 & 0.058378 \\
\hline 12229.34 & 12236.22 & $3.5-3.5$ & 6.873589 & 13.25733 & 0.008973 \\
\hline 12229.65 & 12236.52 & $1.5-1.5$ & 6.870590 & 13.26311 & 0.000008 \\
\hline 12229.55 & 12236.42 & $3.5-3.5$ & 6.868564 & 13.26703 & 0.000957 \\
\hline 12229.65 & 12236.52 & $2.5-1.5$ & 6.867963 & 13.26819 & 0.002244 \\
\hline 12229.21 & 12236.07 & $5.5-4.5$ & 6.865747 & 13.27247 & 0.016528 \\
\hline 12229.51 & 12236.37 & $5.5-4.5$ & 6.865665 & 13.27263 & 0.003575 \\
\hline 12229.56 & 12236.42 & $2.5-3.5$ & 6.864338 & 13.27519 & 0.000011 \\
\hline 12229.34 & 12236.21 & $3.5-4.5$ & 6.862313 & 13.27911 & 0.010704 \\
\hline 12229.24 & 12236.10 & $2.5-1.5$ & 6.861139 & 13.28138 & 0.000048 \\
\hline 12229.14 & 12236.00 & $2.5-2.5$ & 6.860717 & 13.28220 & 0.009757 \\
\hline 12229.57 & 12236.42 & $3.5-3.5$ & 6.853586 & 13.29602 & 0.000320 \\
\hline 12229.57 & 12236.42 & $4.5-3.5$ & 6.850612 & 13.30179 & 0.000004 \\
\hline 12229.43 & 12236.28 & $2.5-1.5$ & 6.848516 & 13.30586 & 0.000017 \\
\hline 12229.16 & 12236.00 & $1.5-2.5$ & 6.847643 & 13.30756 & 0.000003 \\
\hline 12229.63 & 12236.47 & $3.5-2.5$ & 6.839352 & 13.32369 & 0.001630 \\
\hline 12229.38 & 12236.22 & $3.5-3.5$ & 6.834203 & 13.33373 & 0.000092 \\
\hline 12229.24 & 12236.07 & $3.5-4.5$ & 6.834143 & 13.33385 & 0.006647 \\
\hline 12229.37 & 12236.19 & $1.5-2.5$ & 6.825507 & 13.35072 & 0.000004 \\
\hline 12229.65 & 12236.47 & $1.5-2.5$ & 6.825056 & 13.35160 & 0.000016 \\
\hline 12229.69 & 12236.52 & $2.5-1.5$ & 6.824887 & 13.35193 & 0.000152 \\
\hline 12229.38 & 12236.21 & $3.5-4.5$ & 6.822927 & 13.35577 & 0.000185 \\
\hline 12229.65 & 12236.47 & $2.5-2.5$ & 6.822429 & 13.35674 & 0.001915 \\
\hline 12229.46 & 12236.28 & $2.5-1.5$ & 6.820155 & 13.36119 & 0.000007 \\
\hline 12229.55 & 12236.37 & $3.5-4.5$ & 6.819513 & 13.36245 & 0.003215 \\
\hline 12229.66 & 12236.47 & $3.5-2.5$ & 6.815752 & 13.36983 & 0.003481 \\
\hline 12229.28 & 12236.09 & $4.5-5.5$ & 6.813038 & 13.37515 & 0.000059 \\
\hline 12229.57 & 12236.37 & $3.5-4.5$ & 6.804535 & 13.39187 & 0.000037 \\
\hline 12229.57 & 12236.37 & $4.5-4.5$ & 6.801561 & 13.39772 & 0.000020 \\
\hline 12229.72 & 12236.52 & $2.5-1.5$ & 6.801304 & 13.39823 & 0.000894 \\
\hline 12229.47 & 12236.28 & $1.5-1.5$ & 6.801297 & 13.39824 & 0.000003 \\
\hline
\end{tabular}




\begin{tabular}{|c|c|c|c|c|c|}
\hline 12229.48 & 12236.28 & $2.5-1.5$ & 6.791484 & 13.41760 & 0.000001 \\
\hline 12229.43 & 12236.22 & 2.5-3.5 & 6.790663 & 13.41922 & 0.000351 \\
\hline 12229.28 & 12236.07 & 4.5-4.5 & 6.789291 & 13.42193 & 0.003802 \\
\hline 12229.63 & 12236.42 & 3.5-3.5 & 6.788949 & 13.42261 & 0.006245 \\
\hline 12229.14 & 12235.93 & $2.5-1.5$ & 6.788017 & 13.42445 & 0.003072 \\
\hline 12229.43 & 12236.22 & 4.5-3.5 & 6.784212 & 13.43198 & 0.016316 \\
\hline 12229.69 & 12236.47 & $2.5-2.5$ & 6.779353 & 13.44161 & 0.000021 \\
\hline 12229.74 & 12236.52 & 2.5-1.5 & 6.779084 & 13.44214 & 0.002099 \\
\hline 12229.70 & 12236.47 & $3.5-2.5$ & 6.776985 & 13.44631 & 0.008626 \\
\hline 12229.16 & 12235.93 & $1.5-1.5$ & 6.774943 & 13.45036 & 0.000237 \\
\hline 12229.65 & 12236.42 & 4.5-3.5 & 6.774473 & 13.45129 & 0.001489 \\
\hline 12229.43 & 12236.21 & 4.5-4.5 & 6.772936 & 13.45434 & 0.002662 \\
\hline 12229.65 & 12236.42 & 2.5-3.5 & 6.772026 & 13.45615 & 0.000374 \\
\hline 12229.14 & 12235.91 & 2.5-3.5 & 6.770873 & 13.45844 & 0.006515 \\
\hline 12229.75 & 12236.52 & $1.5-1.5$ & 6.770853 & 13.45848 & 0.000007 \\
\hline 12229.24 & 12236.00 & $2.5-2.5$ & 6.766866 & 13.46641 & 0.000056 \\
\hline 12229.24 & 12236.00 & $3.5-2.5$ & 6.766419 & 13.46730 & 0.009722 \\
\hline 12229.43 & 12236.19 & 2.5-2.5 & 6.766021 & 13.46810 & 0.000004 \\
\hline 12229.66 & 12236.42 & 3.5-3.5 & 6.765349 & 13.46943 & 0.002702 \\
\hline 12229.14 & 12235.91 & $2.5-2.5$ & 6.765115 & 13.46990 & 0.007706 \\
\hline 12229.46 & 12236.22 & 2.5-3.5 & 6.762302 & 13.47550 & 0.008199 \\
\hline 12229.51 & 12236.28 & $1.5-1.5$ & 6.762258 & 13.47559 & 0.000123 \\
\hline 12229.72 & 12236.47 & $2.5-2.5$ & 6.755770 & 13.48853 & 0.000001 \\
\hline 12229.16 & 12235.91 & $1.5-2.5$ & 6.752041 & 13.49598 & 0.000015 \\
\hline 12229.53 & 12236.28 & $1.5-1.5$ & 6.744460 & 13.51115 & 0.000602 \\
\hline 12229.63 & 12236.37 & 3.5-4.5 & 6.739898 & 13.52030 & 0.009498 \\
\hline 12229.46 & 12236.19 & 2.5-2.5 & 6.737660 & 13.52479 & 0.000006 \\
\hline 12229.48 & 12236.22 & $2.5-3.5$ & 6.733631 & 13.53288 & 0.000180 \\
\hline 12229.74 & 12236.47 & 2.5-2.5 & 6.733550 & 13.53304 & 0.001282 \\
\hline 12229.37 & 12236.10 & $1.5-1.5$ & 6.730062 & 13.54006 & 0.000205 \\
\hline 12229.69 & 12236.42 & 2.5-3.5 & 6.728950 & 13.54229 & 0.000178 \\
\hline 12229.34 & 12236.07 & $3.5-4.5$ & 6.726953 & 13.54631 & 0.012976 \\
\hline 12229.70 & 12236.42 & 3.5-3.5 & 6.726582 & 13.54706 & 0.000184 \\
\hline 12229.65 & 12236.37 & 4.5-4.5 & 6.725422 & 13.54940 & 0.012606 \\
\hline 12229.75 & 12236.47 & $1.5-2.5$ & 6.725319 & 13.54961 & 0.000014 \\
\hline
\end{tabular}




\begin{tabular}{|c|c|c|c|c|c|}
\hline 12229.70 & 12236.42 & $4.5-3.5$ & 6.718144 & 13.56408 & 0.018115 \\
\hline 12229.56 & 12236.28 & $2.5-1.5$ & 6.717970 & 13.56443 & 0.000010 \\
\hline 12229.66 & 12236.37 & $3.5-4.5$ & 6.716298 & 13.56780 & 0.003112 \\
\hline 12229.48 & 12236.19 & $2.5-2.5$ & 6.708989 & 13.58259 & 0.000016 \\
\hline 12229.72 & 12236.42 & 2.5-3.5 & 6.705367 & 13.58992 & 0.004396 \\
\hline 12229.51 & 12236.21 & 5.5-4.5 & 6.699219 & 13.60239 & 0.000386 \\
\hline 12229.78 & 12236.47 & $3.5-2.5$ & 6.696956 & 13.60699 & 0.000624 \\
\hline 12229.24 & 12235.93 & 2.5-1.5 & 6.694166 & 13.61266 & 0.000263 \\
\hline 12229.38 & 12236.07 & $3.5-4.5$ & 6.687567 & 13.62609 & 0.000058 \\
\hline 12229.74 & 12236.42 & $2.5-3.5$ & 6.683147 & 13.63511 & 0.001705 \\
\hline 12229.70 & 12236.37 & $3.5-4.5$ & 6.677531 & 13.64657 & 0.004265 \\
\hline 12229.24 & 12235.91 & 2.5-3.5 & 6.677022 & 13.64761 & 0.000377 \\
\hline 12229.24 & 12235.91 & $3.5-3.5$ & 6.676575 & 13.64853 & 0.021338 \\
\hline 12229.24 & 12235.91 & 2.5-2.5 & 6.671264 & 13.65939 & 0.000661 \\
\hline 12229.24 & 12235.91 & $3.5-2.5$ & 6.670817 & 13.66031 & 0.010699 \\
\hline 12229.43 & 12236.10 & 2.5-1.5 & 6.670576 & 13.66080 & 0.000041 \\
\hline 12229.70 & 12236.37 & 4.5-4.5 & 6.669093 & 13.66384 & 0.001371 \\
\hline 12229.55 & 12236.22 & 3.5-3.5 & 6.664343 & 13.67358 & 0.021111 \\
\hline 12229.53 & 12236.19 & $1.5-2.5$ & 6.661965 & 13.67846 & 0.000003 \\
\hline 12229.43 & 12236.09 & 4.5-5.5 & 6.661323 & 13.67978 & 0.003991 \\
\hline 12229.86 & 12236.52 & $1.5-1.5$ & 6.660873 & 13.68070 & 0.000968 \\
\hline 12229.56 & 12236.22 & 2.5-3.5 & 6.660117 & 13.68225 & 0.005373 \\
\hline 12229.34 & 12236.00 & $3.5-2.5$ & 6.659229 & 13.68408 & 0.001049 \\
\hline 12229.55 & 12236.21 & $3.5-4.5$ & 6.653067 & 13.69675 & 0.055440 \\
\hline 12229.57 & 12236.22 & 3.5-3.5 & 6.649365 & 13.70438 & 0.020415 \\
\hline 12229.78 & 12236.42 & 3.5-3.5 & 6.646553 & 13.71018 & 0.000115 \\
\hline 12229.57 & 12236.22 & 4.5-3.5 & 6.646391 & 13.71051 & 0.000039 \\
\hline 12229.46 & 12236.10 & $2.5-1.5$ & 6.642215 & 13.71913 & 0.000399 \\
\hline 12229.55 & 12236.19 & $3.5-2.5$ & 6.639701 & 13.72433 & 0.000015 \\
\hline 12229.57 & 12236.21 & 3.5-4.5 & 6.638089 & 13.72766 & 0.033137 \\
\hline 12229.43 & 12236.07 & 4.5-4.5 & 6.637576 & 13.72872 & 0.034227 \\
\hline 12229.37 & 12236.00 & $1.5-2.5$ & 6.635789 & 13.73242 & 0.001830 \\
\hline 12229.56 & 12236.19 & $2.5-2.5$ & 6.635475 & 13.73307 & 0.000007 \\
\hline 12229.57 & 12236.21 & 4.5-4.5 & 6.635115 & 13.73381 & 0.008751 \\
\hline 12229.28 & 12235.91 & 4.5-3.5 & 6.631723 & 13.74084 & 0.000058 \\
\hline
\end{tabular}




\begin{tabular}{|l|l|l|l|l|l|}
\hline 12229.65 & 12236.28 & $2.5-1.5$ & 6.625658 & 13.75341 & 0.000033 \\
\hline 12229.57 & 12236.19 & $3.5-2.5$ & 6.624723 & 13.75536 & 0.000007 \\
\hline 12229.47 & 12236.10 & $1.5-1.5$ & 6.623357 & 13.75819 & 0.000004 \\
\hline 12229.38 & 12236.00 & $3.5-2.5$ & 6.619843 & 13.76550 & 0.000011 \\
\hline 12229.58 & 12236.19 & $1.5-2.5$ & 6.616343 & 13.77278 & 0.000002 \\
\hline
\end{tabular}

Table C 8: Calculation of Sn XIII $4 \mathrm{~d}^{2}-4 \mathrm{~d}^{1} 4 \mathrm{f}^{1}$ EUV transitions.

\begin{tabular}{|c|c|c|c|c|c|}
\hline Lower level, Ry & Upper level, Ry & $J_{i}-J_{j}$ & Energy, Ry & $\lambda, \mathrm{nm}$ & $f_{i j}$ \\
\hline 12212.30 & 12219.24 & $5-4$ & 6.945076 & 13.12087 & 0.028954 \\
\hline 12212.67 & 12219.41 & $3-2$ & 6.740982 & 13.51812 & 0.230132 \\
\hline 12212.42 & 12219.14 & $1-2$ & 6.728113 & 13.54398 & 0.040631 \\
\hline 12212.30 & 12219.00 & $5-4$ & 6.704292 & 13.59210 & 0.721355 \\
\hline 12212.75 & 12219.41 & $2-2$ & 6.658619 & 13.68533 & 0.012508 \\
\hline 12212.67 & 12219.32 & $3-3$ & 6.655111 & 13.69255 & 0.007450 \\
\hline 12212.80 & 12219.41 & $3-2$ & 6.605690 & 13.79499 & 0.113100 \\
\hline
\end{tabular}

Table C 9: Calculation of Sn XIII $4 d^{2}-4 d^{1} 5 p^{1}$ EUV transitions.

\begin{tabular}{|c|c|c|c|c|c|}
\hline Lower level, Ry & Upper level, Ry & $J_{i}-J_{j}$ & Energy, Ry & $\lambda, \mathrm{nm}$ & $f_{i j}$ \\
\hline 12212.10 & 12219.01 & $2-2$ & 6.912305 & 13.18307 & 0.002445 \\
\hline 12212.13 & 12219.01 & $2-2$ & 6.878340 & 13.24817 & 0.041117 \\
\hline 12212.27 & 12219.14 & $1-2$ & 6.869359 & 13.26549 & 0.000677 \\
\hline 12212.27 & 12219.01 & $1-2$ & 6.736806 & 13.52650 & 0.019506 \\
\hline
\end{tabular}


Distribution List for ANL-ET-04/24

Internal:

J. P. Allain

J. N. Brooks

A. Foley

D. Gruen

J. Harmon

A. Hassanein (10)

Z. Insepov

I. Konkashbaev

V. Morozov (5)

J. Norem

R. B. Poeppel

W. Shack

V. A. Sizyuk

S. W. Tam

TIS Files

External:

DOE-SC (5)

ANL Libraries:

$$
\text { ANL-E }
$$

Main Library:

Lawrence Berkeley National Laboratory

Lawrence Livermore National Laboratory

Los Alamos National Laboratory

Naval Research Laboratory

Sandia National Laboratories

Energy Technology Division Review Committee:

M.L. Corradini, University of Wisconsin, Madison, WI

J.M. Deutch, Massachusetts Institute of Technology, Cambridge, MA

N.G. Eror, University of Pittsburgh, Pittsburgh, PA

C. Logan, Lawrence Livermore National Laboratory, Livermore, CA

M.A. Ulrickson, Sandia National Laboratories, Albuquerque, NM

R. Zoughi, University of Missouri-Rolla, Rolla, MO

J.C. Davis, retired from LLNL, 2262 Buena Vista Avenue, Livermore, CA 
External:

M. Al-Rabban, Physics Department, Qatar University, Doha, Qatar

R. Anderson, Sandia National Laboratory, Albuquerque, NM

V. Bakshi, International SEMATECH, Austin, TX

D. Bolshukhin, XTREME technologies GmbH, Jena, Germany

R. Bristol, Intel Corporation, Santa Clara, CA

C. Bruineman, FOM Institute for Plasma Physics Rijnhuizen, Nieuwegein, The Netherlands

P. Choi, EPPRA SAS, Courtaboeuf, France

G. Derra, Philips GmbH, Forschungslaboratorien, Aachen, Germany

A. Egbert, Laser Zentrum Hannover, Hannover, Germany

A. Endo, Extreme Ultraviolet Lithography System Development Association, Kanagawa, Japan

S. Ellwi, PowerLase Limited, West Sussex, UK

B. Fay, Alcatel Vacuum Technology, Annecy, France

I. Fomenkov, Cymer, Inc., San Diego, CA

K. Gäbel, XTREME technologies GmbH, Jena, Germany

J. Gillaspy, National Institute of Standards and Technology, Gaithersburg, MD

S. Grantham, National Institute of Standards and Technology, Gaithersburg, MD

J. Greenwood, BOC Edwards, West Sussex, UK

Y. Izawa, Institute of Laser Engineering, Osaka University, Suita, Osaka, Japan

C-S. Koay, University of Central Florida, Orlando, FL

T. Kawamura, Institute for Laser Technology, Osaka, Japan

K. Koshelev, Institute of Spectroskopy, RAS, Troitsk, Russia

T. Kumazaki, Komatsu Ltd, Hiratsuka-shi, Kanagawa, Japan

R. Lebert, AIXUV GmbH, Aachen, Germany

E. Loius, FOM Institute for Plasma Physics Rijnhuizen, Nieuwegein, The Netherlands

K. Mann, Laser-Laboratorium Göttingen, Göttingen, Germany

P. Marczuk, Carl Zeiss Laser Optics GmbH, Oberkochen, Germany

M. McGeoch, PLEX LLC, Brookline, MA

H. Milchberg, University of Maryland, College Park, MD

T. Mochizuki, Himeji Institute of Technology, Kamigori, Akou, Hyogo, Japan

K. Nishihara, Institute of Laser Engineering, Osaka University, Suita, Osaka, Japan

T. Nishikawa, Faculty of Engineering, Okayama University, Okayama, Japan

J. Pankert, Philips Extreme UV GmbH, Aachen, Germany

J. Pomeroy, National Institute of Standards and Technology, Gaithersburg, MD

C. Rettig, Cymer, Inc., San Diego, CA

J. Ricardi, JMAR Technologies, Inc., San Diego, CA 
B. Rice, Technology Development, Intel Corporation, Hillsboro, OR

M. Richardson, University of Central Florida, Orlando, FL

A. Sasaki, Advance Photon Research Center, JAERI-KANSAI, Kizu, Kyoto, Japan

M. Schuermann, JENOPTIK Mikrotechnik GmbH, Jena, Germany

F. Scholze, Physikalish-Technische Bundesanstalt, Berlin, Germany

L. Shmaenok, PhysTex, Nieuwegein, The Netherlands

U. Stamm, XTREME technology GmbH, Jena, Germany

K. Takenoshita, University of Central Florida, Orlando, FL

Y. Teramoto, EUVA, Gotenba, Shizuoka, Japan

Ch. Walton, Lawrence Livermore National Laboratory, Livermore, CA

Y. Watanabe, Canon Inc., Utsunomiya-city, Japan

O. Wood, International SEMATECH, Austin, TX

Ch. Ziener, XTREME technologies GmbH, Jena, Germany

Bibliothek, Max-Planck-Institute für Plasmaphysik, Germany

C.E.A. Library, Fontenay-aux-Roses, France

Librarian, Culham Laboratory, England

Thermonuclear Library, Japan Atomic Energy Research Institute, Japan

University of Wisconsin Library 